\title{
Structural, functional and metabolic aspects of shortening and lengthening muscle contractions
}

Citation for published version (APA):

Hesselink, M. K. C. (1998). Structural, functional and metabolic aspects of shortening and lengthening muscle contractions. [Doctoral Thesis, Maastricht University]. Universiteit Maastricht. https://doi.org/10.26481/dis.19980312mh

Document status and date:

Published: 01/01/1998

DOI:

10.26481/dis.19980312mh

Document Version:

Publisher's PDF, also known as Version of record

\section{Please check the document version of this publication:}

- A submitted manuscript is the version of the article upon submission and before peer-review. There can be important differences between the submitted version and the official published version of record.

People interested in the research are advised to contact the author for the final version of the publication, or visit the DOI to the publisher's website.

- The final author version and the galley proof are versions of the publication after peer review.

- The final published version features the final layout of the paper including the volume, issue and page numbers.

Link to publication

\footnotetext{
General rights rights.

- You may freely distribute the URL identifying the publication in the public portal. please follow below link for the End User Agreement:

www.umlib.nl/taverne-license

Take down policy

If you believe that this document breaches copyright please contact us at:

repository@maastrichtuniversity.nl

providing details and we will investigate your claim.
}

Copyright and moral rights for the publications made accessible in the public portal are retained by the authors and/or other copyright owners and it is a condition of accessing publications that users recognise and abide by the legal requirements associated with these

- Users may download and print one copy of any publication from the public portal for the purpose of private study or research.

- You may not further distribute the material or use it for any profit-making activity or commercial gain

If the publication is distributed under the terms of Article $25 \mathrm{fa}$ of the Dutch Copyright Act, indicated by the "Taverne" license above, 


\section{CIP-GEGEVENS KONINKLIJKE BIBLIOTHEEK, DEN HAAG}

Hesselink, Matthijs Karel Christiaan

Structural, functional and metabolic aspects of shortening and lengthening muscle contractions / Matthiijs K.C. Hesselink

Maastricht: Universitaire Pers Maastricht - III

Proefschrift Maastricht - Met lit. opg. -

Met samenvatting in het Nederlands

ISBN 90-9011401-7

Cover: Photomicrographs of transverse muscle sections taken 24 hours after shortening exercise (left panel) and lengthening exercise (right panel).

Vormgeving: Matthijs Hesselink

Omslagidee: Matthijs Hesselink

Druk: Datawyse Maastricht/ Krips Repro Meppel

(C) 1998 Hesselink, Maastricht, The Netherlands

Printing of this thesis was financially supported by the Dr. Ir. J.H. van der Laar Stichting. 


\title{
Structural, Functional and Metabolic Aspects of Shortening and Lengthening Muscle Contractions
}

\author{
PROEFSCHRIFT
}

ter verkrijging van de graad van doctor aan de Universiteit Maastricht,

op gezag van de Rector Magnificus, Prof. dr. A.C. Nieuwenhuijzen Kruseman, volgens het besluit van het College van Decanen, in het openbaar te verdedigen op donderdag 12 maart 1998 om 16.00 uur

door

\section{Matthijs Karel Christiaan Hesselink}

geboren te Bemmel op 8 augustus 1968 


\section{Promotor:}

Prof. dr. H. Kuipers

\section{Co-promotores:}

Dr. H.A. Keizer

Dr. ir. M.R. Drost

\section{Beoordelingscommissie:}

Prof. dr. ir. W.H.M. Saris (voorzitter)

Prof. dr. F.C.S. Ramaekers

Dr. H.H.C.M. Savelberg

Prof. dr. G.J. van der Vusse

Dr. A.J.M. Wagenmakers 


\section{Table of Contents}

Page

Chapter 1 General Introduction

Chapter 2 Characterization and Testing of the Experimental

Set-Up and Exercise Protocols Used

Chapter 3 Structural Muscle Damage and Muscle Strength After Incremental Number of Isometric and Forced Lengthening Contractions

Chapter $4 \quad$ Acute and Sustained Effects of Isometric and Lengthening Muscle Contractions on High-Energy Phosphates and Glycogen Metabolism in Rat Tibialis Anterior Muscle

Chapter 5 Muscle Glycogen Following Lengthening and Shortening Muscle Contractions in Relation to Activity of Glycogen Regulatory Enzymes and Muscle GLUT4 Content

Chapter 6 Immunolocalization of GLUT4 at Light Microscopical Level in Rat Cardiac and White Gastrocnemius Muscle

Chapter $7 \quad$ Increased Contractile Work During Lengthening

Contractions After Shortening and Lengthening Training

Chapter $8 \quad$ Changes in the Expression of Myofibrillar and Cytoskeletal Proteins Following Shortening and Repeated Lengthening Exercise

Chapter 9 General Discussion

Summary

Samenvatting

Dankwoord

Curriculum vitae

Publications 



\section{General Introduction}




\section{Introduction}

In human adults, skeletal muscle comprises approximately $40 \%$ of total body mass. The most prominent function of skeletal muscle is to enable active interaction with the environment. Due to a highly organized structure, including contractile proteins, the muscle can contract and initiate movements. Essential for movement is the conversion of chemical energy into mechanical energy during muscle contraction. The chemical energy needed for muscle contraction is provided by hydrolysis of ATP, which is predominantly synthesized from breakdown of carbohydrates and fatty acids. Compared to the resting state, breakdown of carbohydrates and fatty acids is dramatically increased in active muscles.

Muscle is characterized by a great plasticity enabling adjustment to changes in physiological demands. With regular physical exercise, muscular adaptations take place, permitting the exercising muscle to better cope with the increased demands of work. Such adaptations involve alterations in energy metabolism (e.g., a relative increased fatty-acid oxidation at high-intensity exercise after endurance training) as well as adaptations in the contractile apparatus itself (e.g., increased muscle volume after strength training). The extent and direction of such adaptations depend among others on the duration, frequency and type of exercise performed (e.g., endurance or strength training). Another factor that affects the extent and direction of adaptations of muscle tissue is the nature of contraction performed by the muscle. In general 3 types of muscle contraction are distinguished, (1) isometric contractions in which both muscle ends remain in position and gross muscle length does not change, (2) concentric contractions in which the muscle shortens and (3) eccentric contractions when a force external to the muscle overcomes muscle contractile force and muscles are lengthened. The latter two types of muscle contraction will be referred to as shortening and lengthening contractions, respectively.

Although physical exercise training generally improves muscie function, unaccustomed or lengthening exercise may induce structural damage to the exercised muscles. In turn, muscle function following lengthening exercise is compromised. The mechanism underlying compromised muscle function after damaging lengthening exercise is not completely understood. Therefore, in the present thesis, functional and metabolic aspects of shortening and lengthening muscle contractions are studied. For a proper understanding of functional and metabolic aspects of muscle contraction, this chapter starts with a brief description of skeletal muscle with regard to structure and function. The research objective of the thesis is outlined in more detail at the end of this chapter.

\section{Skeletal muscle structure}

Architectural gross structure of skeletal muscle

A muscle consists of several muscle cells, which are fused cells forming nucleated tubes, also referred to as muscle fibers. Intact muscles are surrounded by a connective tissue sheet named epimysium or fascia. When looking at a cross section of intact muscle, bundles of muscle fibers can be distinguished that are also surrounded by a connective tissue sheet (perimysium). Within these bundles of fibers, individual myofibrils are found, surrounded by a thin connective tissue sheet 
as well (endomysium). For the supply of nutrients, oxygen and for the drainage of metabolites, a network of blood vessels exists and capillaries can be found around the myofibrils. In transverse sections of healthy skeletal muscle, polygonal shaped, multinucleated muscle fibers are visible. In healthy skeletal muscle, nuclei are found at the cell border and not in the cell interior.

Originally, myotubes were believed to span the entire distance between proximal and distal tendons. To date, reports are available showing the existence of tapering fibers, arranged end-to-end in series within a fascicle, so called 'series fibered muscles' (36). Introduction of the microscope by van Leeuwenhoek in the 1670 s revealed that in longitudinal sections, skeletal muscle possesses a banded appearance and is therefore also called striated muscle. The banding pattern of striated muscle originates from the regular arrangement of the contractile proteins within the muscle fiber.
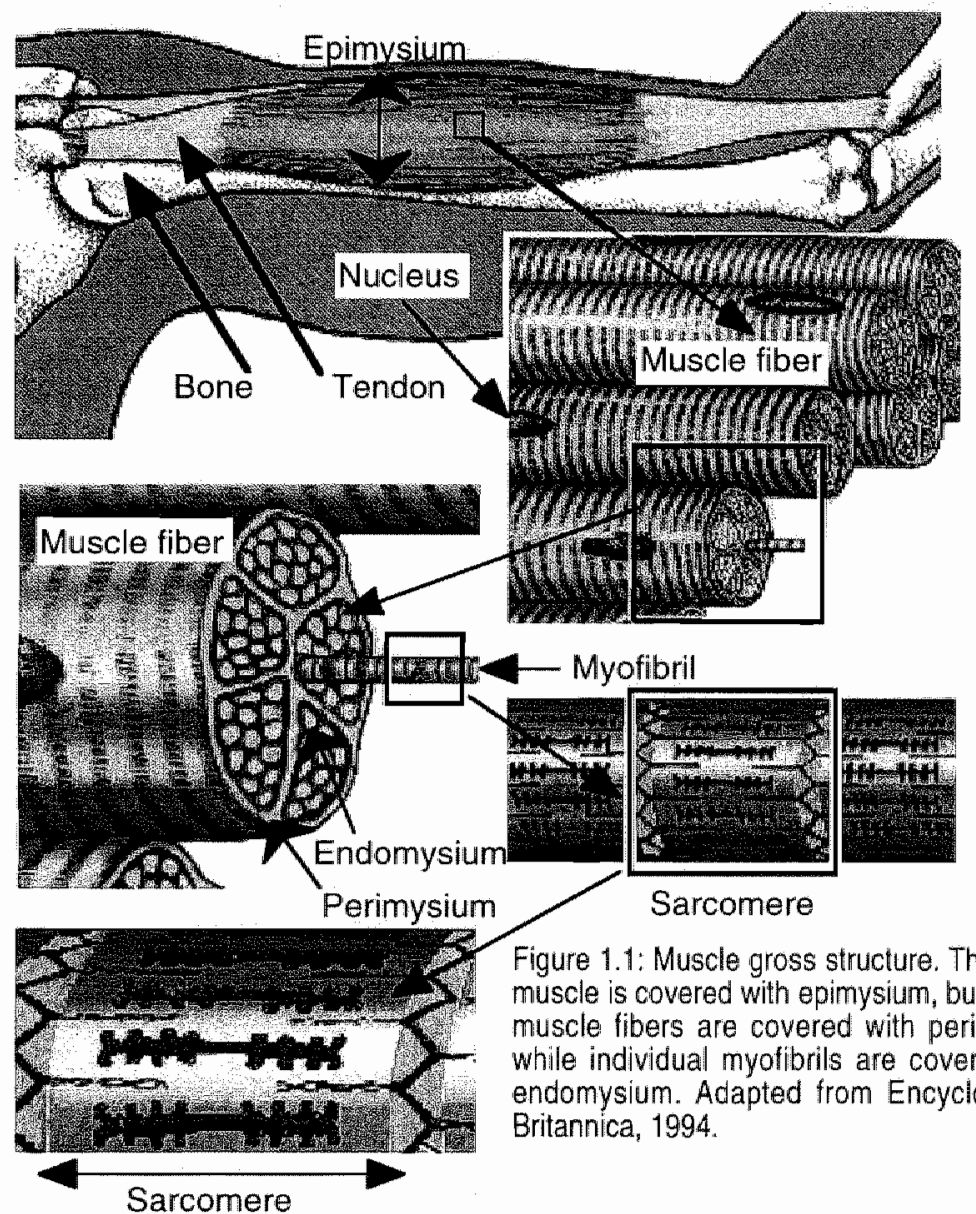

Figure 1.1: Muscle gross structure. The entire muscle is covered with epimysium, bundles of muscle fibers are covered with perimysium while individual myofibrils are covered with endomysium. Adapted from Encyclopaedia Britannica, 1994. 


\section{Muscle fiber constituents and its fine structure}

Within the muscle fiber the contractile proteins actin and myosin make up the majority of total protein content (22 and $44 \%$, respectively) (1). In resting muscle, actin and myosin partly overlap and interaction of actin-myosin is the bases for muscle contraction.

Actin is a globular protein, also referred to as thin filament. At one end actin filaments attach to the so-called $Z$-discs in a hexagonal array. Actin is associated with a long fibrous protein, tropomyosin, which in turn bears a protein complex of 3 subunits of troponin (troponin I, troponin $\mathrm{C}$ and troponin T). Together, the troponin subunits regulate binding of myosin heads to tropomyosin by conformational changes in the presence of calcium lons (1), the triggers of muscle contraction.

Myosin, also referred to as thick filaments, consists of a tail region of 4 heavy myosin chains and a double globular head at the end, consisting of 2 myosin light chains. The tail region of myosin makes up the thick filament structure while the globular heads have a regulatory role in calcium binding and subsequent actin binding. The globular head has an ATP molecule bound in a cleft and contains the ATP hydrolyzing enzyme ATP-ase. Thick filaments are helically arranged and the 2 tail regions are facing each other and globular heads can act with tropomyosin in a radial fashion. Since the tails are facing, the thick filaments are symmetrical about the center where a bare zone with no globular heads is present ( $\mathrm{H}$-zone).

Sarcomere architecture is schematically depicted in figure 2 . The wide, dark, zone is referred to as $\mathrm{A}$-band and covers the entire length of a myosin molecule, in the middle of the $\mathrm{A}$ band the $\mathrm{H}$-zone is present, representing the bare zone of the thick filament. Central in the $\mathrm{H}$-zone, the $\mathrm{M}$-line is observed (containing $\mathrm{M}$-protein, myomesin and the muscle isoform of creatine kinase). Between 2 adjacent Abands the 1-band is present (mainly consisting of actin), in the middle of the I band, actin molecules are connected to the Z-discs.

The architecture of sarcomeres is maintained by cytoskeletal proteins keeping the contractile proteins in the correct spatial arrangement for force production by making serial and lateral connections between myofibrils. Both endo- and exosarcomeric cytoskeletal proteins are present in skeletal muscle.

Within the sarcomere, actin is linked to the Z-disc by $\alpha$-actimin (21). Another large actin associated protein is nebulin, whose putative function is to regulate actin length $(31,64)$. The distance between $Z$-disc and M-line is spanned by titin, a giant protein with an elastic and a mon-elastic region maintaining myosin alignment and supporting passive tension $(31,68)$.

The exosarcomeric lattice is composed of intermediate filaments of which desmin is the most prominent protein present in mature skeletal muscle (32). Desmin links adjacent $Z$-discs in a longitudinal and lateral fashion and is responsible for the almost perfect register of adjacent Z-discs and A bands. During early muscle cell differentiation desmin is co-expressed with vimentin, synemin and paranemin (49). The cytoskeleton is anchored to the sarcolemma by costameres. Based on in vitro binding studies of specific proteins, combined with structural information. 5 distinct proteins (actin, $\alpha$-actinin, vinculin, talin and dystrophin) are hypothesized to play a functional role in the connection of the cytoskeleton to the 
sarcolemma by costameres (46). Consequently, these proteins will play a role in force transmission to the endomysium.

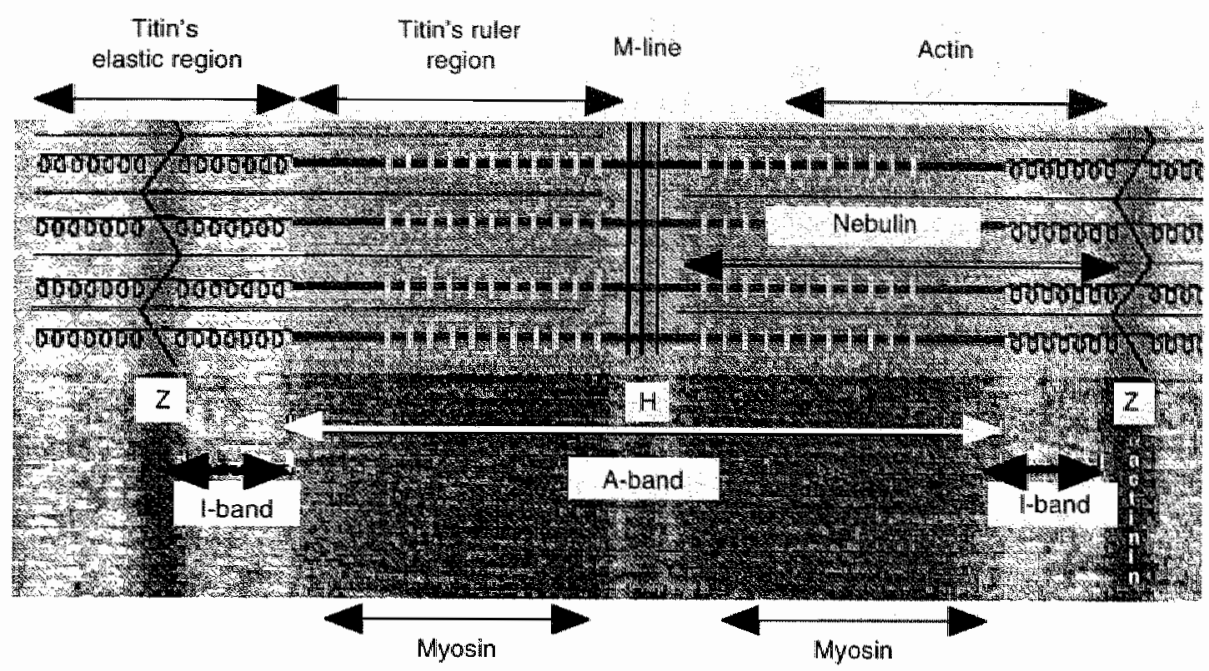

Figure 1.2: Electron photomicrograph of a single sarcomere. The distance between 2 Z-lines approximates $2.3 \mu \mathrm{m}$.

\section{Force production of skeletal muscle}

Nowadays, the sliding filament model proposed by A.F. Huxley in the late 50 s has become a widely accepted model for force generation by actomyosin interaction (27).

Actomyosin interaction is triggered by release of calcium from the sarcoplasmic reticulum. Binding of calcium to troponin $\mathrm{C}$ induces conformational changes in troponin $T$ and troponin I, subsequently the actin binding sites of troponin are exposed and the globular heads of myosin may bind to actin.

Before binding to actin, ATP bound in the cleft of the globular head of myosin is split by ATP-ase into ADP and phosphate (P) and retains in the ATP-ase site until the globular head attaches to actin. Upon attachment, $P$ is released and the head is tightly connected to actin and undergoes a conformational change that makes the head bend and actin shifts towards the center of the sarcomere, the so-called 'power stroke'. Detachment of the globular head only occurs after the release of ADP and binding of a new ATP after which the globular head is ready for the next attachment to actin. The complete cycle of actomyosin interaction is referred to as a 'cross bridge cycle'. Since ATP is essential for cross bridge detachment, ATP levels must be maintained throughout exercise.

Force generation during shortening and lengthening contractions

The tension produced during muscle contraction depends on muscle intrinsic properties, sarcomere length and contractile velocity together with the nature of the contraction (shortening, isometric or lengthening). Here we will restrict the 
discussion to force generation in relation to muscle length and contractile velocity during shortening and lengthening muscle contractions.

According to the sliding filament model, force is generated by each individual cross bridge cycle within a sarcomere. Consequently, the amount of force produced is related to the number of cross bridges. The number of cross bridges depends on the extent to which actin and myosin are overlapped, which in turn depends on muscle length. Functionally, the dependency of muscle force generation on muscle length is indicated by the existence of an optimal muscle (sarcomere) length ( $\left.L_{0}\right)$. At muscle lengths on either side of $L_{0}$, less force is produced.

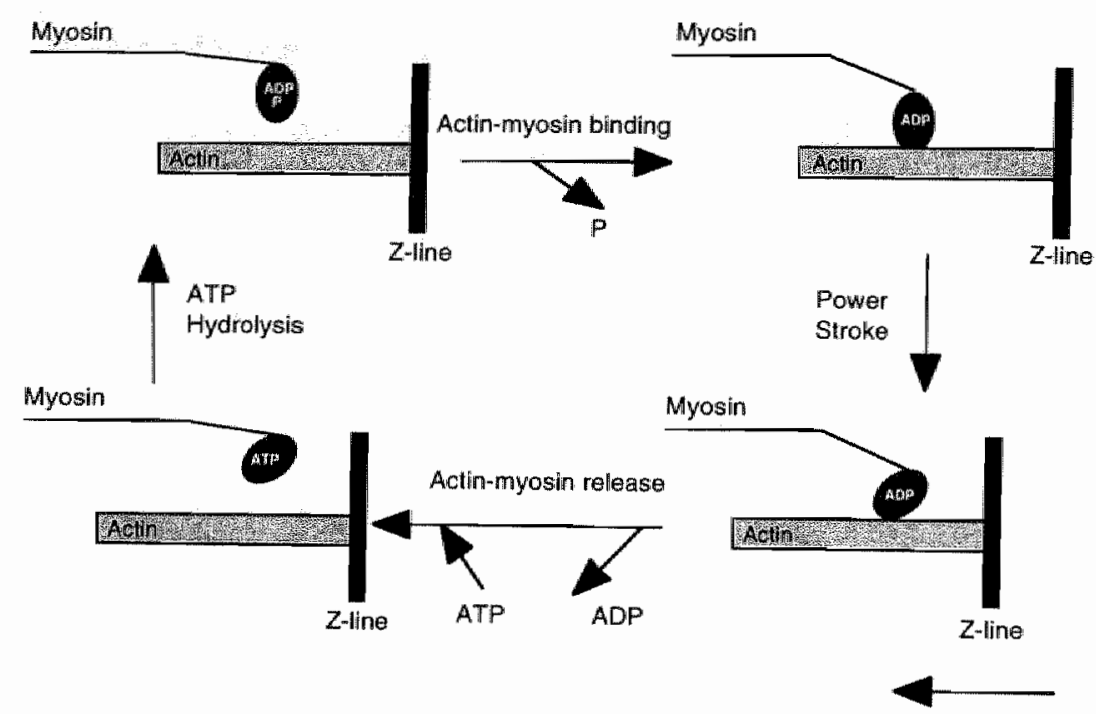

Figure 1.3: Cross-bridge cycle. Upon hydrolysis of ATP, bound in the cleft of the globular head of actin, the myosin head is ready to bind to actin. After binding, actin undergoes a conformational change, initiating the 'Power stroke'. Detachment of myosin occurs after release of ADP' and binding of a new ATP. Subsequently, a next cross-bridge cycle can take place.

During shortening muscle contraction, less force is produced when contractile velocity increases. The number of cross bridges attached decreases at high velocity, simply due to the fact that at high shortening velocities, only little time is available for cross bridge attachment. Considerably more force can be produced during lengthening than during shortening exercise and force plateaus at a higher contractile velocity. The greater amount of force production while lengthening a muscle is assumed to originate from the compliant S2 portion of myosin light chains that is stretched further during lengthening than during shortening contractions (28). With increasing stretching speed less cross bridges will be attached. Consequently, those attached will sustain more force and force production plateaus when contractile velocity increases (28). 


\section{Force transmission in skeletal muscle}

After force is produced at the cross bridge level, force transmission to the skeletion is required for locomotion. It is well accepted that transmission of force occurs serially throughout the myofibrils. To date, increasing evidence has become available that lateral force transmission from adjacent sarcomeres via an intermediate filament network to costameres connected to the sarcolemma plays a substantial role in total force transmission (46). Upon transmission of force to the sarcolemma, it is transferred to the endomysium by the extracellular matrix and finally to the muscle-tendon complex.

Lateral force transmission between adjacent muscle fibers was elegantly shown by Street (62) who dissected a single myofiber at one end of the muscle and left the surrounding fibers intact. In one experimental condition the tendon of the muscle was fixed to a force transducer and the bare end of the single fiber was anchored as well, while the remaining muscle fibers were untethered. In the other experimental condition the tendon was again attached to the force transducer and the end of the single fiber was untethered while the remaining fibers were fixed. In both experimental conditions the bare end of the single fiber was stimulated and recorded force was essentially the same (62).

Once force is transmitted from the contractile elements to the tendon attached to the skeleton, torque is produced around the joint spanned by the active muscle. When the torque produced equals the opposing torque (from for example antagonist muscle activity or gravity) no movement will come about (isometric contraction), consequently no external work is performed by the muscle. If the torque produced by the muscle overcomes the opposed torque, the muscle will shorten and work is produced (shortening contraction). If the opposite torque exceeds muscle torque, the muscle will lengthen and work will be absorbed by the muscle (lengthening contraction).

\section{Fueling force generation}

According to Huxley's 1957 sliding filament theory one ATP is hydrolyzed during every cross bridge cycle (27). In general, when contractile force increases the number of cross bridges attached increases, consequently ATP hydrolysis is enhanced when force development increases. However, during lengthening contractions more force is produced per molecule hydrolyzed ATP than during shortening contractions $(11,19)$. The lower rate of ATP splitting during lengthening may be due to the fact that during lengthening, detachment of cross-bridges is primarily a mechanical process (28). Maintenance of adequate ATP levels throughout both lengthening and shortening exercise is essential for muscle performance.

ATP maintenance in skeletal muscle

In most mammalian cells, like skeletal muscle, ATP levels are maintained by oxidative phosphorylation in the mitochondrial inner membrane. During muscle contraction the myofilaments are the major site for ATP hydrolysis. The susbstantial increase in ATP demand during exercise requires rapid rephosphorylation of ADP. Therefore, transport of the relatively large and charged ATP molecule from mitochondria to the myosin ATP-ase would be ineffective. This problem is 
overcome by the presence of a so called "energy shuttle" in muscle (67). In the mitochondrial inner membrane the energy-rich phosphate of ATP is transferred to creatine in a reaction catalyzed by the mitochondrial isoform of creatine kinase, leading to the formation of phosphocreatine (PC). Transport of PC to myosin ATP. ase is rapid and $A D P$ and $P C$ are readily converted to $A T P$ and creatine in a reaction catalyzed by the muscle isoform of creatine kinase, present in the M-line $(67)$.

Mitochondrial ATP production occurs by reducing agents (NADH and FADH2) formed in the tricarboxylic acid cycle (TCA-cycle). The TCA-cycle is fed with carbon atoms of which the vast majority is derived from acetyl-CoA, which in turn originates either from pyruvate formed during the breakdown of carbohydrates in glycollysis, or from breakdown of fatty acids by B-oxidation.

Breakdown of carbohydrates in glycolysis is the major pathway for ATP synthesis during high-intensity exercise. Carbohydrates can be taken up from the blood as glucose or can be derived from the intracellular storage form of glucose, muscle glycogen. When intense exercise is prolonged, muscle glycogen stores will decline and the capacity to generate contractile work declines.

Oxidation of fatty acids is the major pathway for acetyl-CoA synthesis and subsequent ATP generation under resting conditions, during exercise when gllycogen stores are depleted or during relatively low intensity exercise. Fatty acids are derived from triacylglycerol which is stored in large, virtually inexhaustible, amounts in adipose tissue and to a lesser extent in the skeletal muscle.

Although glucose and fatty acid metabolism are presented here as two distinct mechanisms, they are interacting in close harmony in living vertebrates and neither one is ever completely 'switched off'. Interaction of glucose and fatty acid metabolism is generally believed to operate via the glucose-fatty acid cycle (50). To date, evidence is growing that mallonyl-CoA plays a pivatal role in interaction of glucose and fatty acid metabolism (14-16, 44, 48, 53, 72). Amino acid oxidation may also help to maintain ATP levels, however in exercising healthy muscle its role is quantitatively less important.

\section{Muscle damage}

Muscle fibers can be damaged in numerous ways including crush injuries burn injuries, injury due to immobilization, decubitis ulcers and exercise-induced muscle
damage.

Exercise-induced muscle damagle may originate from unaccustomed exercise as well as from exercise involving lengthening contractions. In the present thesis, when talking about muscle damage, we are referring to damage induced by
lengthening contractions.

In muscles damaged by lengthening exercise, declined muscle performance is a common observation $(37,38,51,52)$. Declined muscle performance following damage is accompanied by metabolic alterations $(18,26,47,57,60,66)$. Structural abnormalities have been reported as well, including sarcolemmal disruption (2, 8 ,

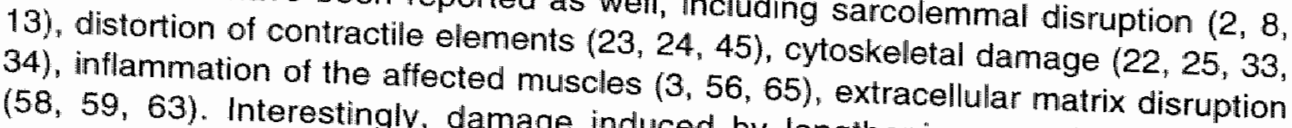
$(58,59,63)$. Interestingly, damage induced by lengthening exercise is quickly 
reversible $(4,7,17)$ and when a muscle is subjected to lengthening contractions again, previously lengthened muscles are less vulnerable to lengthening exerciseinduced damage $(17,39,57)$.

Histologically observed muscle damage is not directly related to the number of contractions performed (37). However, if muscle damage is defined as the percentage decline in concentric force after low-intensity lengthening contractions, muscle damage increases with the number of contractions (61). Therefore, it appears that muscle force is not essentially related to the area of histological muscle injury $(33,35,69,70)$. At present, force decline following damaging exercise and its relation to the extent of histologically observed damage remains unclear.

Besides the likely contribution of structural damage of the contractile apparatus, also metabolic alterations may depress muscle contractile performance. Reports exist on delayed and hampered glycogen resynthesis in damaged muscles $(10,12,30,43,66)$. Unsulin resistance following lengthening exercise may contribute to the depressed glycogen resynthesis following lengthening exercise $(20,29,55)$. Insulin resistance in damaged muscle may originate from declined expression of the insullin and contraction regulatable glucose transporter 4 (GLUT4) $24 \mathrm{~h}$ after damaging exercise $(5,6)$. In healthy skeletal muscle, glycogen is restored to pre-exercise levels within $6 \mathrm{~h}$. However, reports on restoration of muscle glycogen during the first 6 hour following damaging exercise are scarce (71) and the role of GLUT4 during the rapid restoration phase of muscle glycogen after damaging lengthening exercise, remains unclear.

The mechanism underlying the rapid adaptive response of previously lengthened muscles to lengthening exercise $(42,54)$ is poorly understood. Blunted enzyme release, less soreness, and less pronounced changes in relaxed elbow angle are the most commonlly reported findings following repeated lengthening exercise (9). In most cases when muscle performance was assessed following repeated lengthening exercise, maximal voluntary contractile force was recorded $(39,41,42)$. Reports on muscle performance following repeated lengthening exercise under standardized and controlled conditions are scarce. Muscle damage induced remodeling of the force-producing and transmitting network has been hypothesized to play a role in muscle performance following damaging exercise (46) and may be related to the less compromised muscle function following repeated lengthening exercise.

\section{Aim and outline of the present thesis}

The central aim of the research reported in the present thesis was to investigate the effect of different types of muscle contraction on muscle force, muscle work, myofibrillar and cytoskeletal proteins and energy metabolism.

In chapter 2 we describe a dynamometer constructed to record contractile work of rat hindllimb muscles in vivo, the chapter aims to provide a detailed description of the characteristics of this dynamometer and validates the experimental procedures used throughout this thesis. In chapter 3 we aimed to assess the relationship between the number of lengthening contractions and the extent of muscle damage and concomitant force decline. Chapter 4 we aimed to 
study the effect of damaging and non-damaging exercise on acute and prolonged alterations in energy metabolism. In chapter 5 we aimed to study the mechanism behind hampered resynthesis of muscle glycogen, as observed in earlier studies. In chapter 6 it was studied whether immunolocalization of GLUT4 at light microscopical level would provide information of sufficient detail to study insulin and contraction-induced translocation of GLUT4. In chapter 7 we studied whether the amount of contractile work performed during a lengthening exercise session changed when the lengthening exercise session was repeated within 2 weeks. Furthermore, we aimed to study the effect of repeated lengthening exercise on the extent of muscle damage. In chapter 8 the expression of myofibrillar and cytoskeletal proteins following shortening exercise and repeated lengthening exercise was investigated by one- and two-dimensional gel electrophoresis and immunoblotting. Finally, in chapter 9 , the major findings of the research project described in the present thesis are discussed. 


\section{References}

1. Alberts, B, D. Bray, J. Lewis, M. Raff, K. Roberts, and d. D. Watson. Molecular biology of the cell New York \& London: Garland publishing, Inc., 1989.

2. Armsirong, R. B. Initial events in exercise-induced muscular injury. Med Sci Sports Exerc 22: 429 435, 1990.

3. Armstrong, I. B., R. W. Oglivie, and J. A. Schwane. Eccentric exercise induced injury to skeletal muscle. d Appl Physiol 54: 80-93, 1983.

4. Armstrong, R. B. G. L. Warren, and J. A. Warren. Mechanisms of exercise-induced muscle fiber injury. Sports Med 12: 184-207, 1991

5. Asp, S., J. R. Daugaard, S. Kristiansen, B. Kiens, and E. A. Richter. Eccentric exercise decreases maximal insulin action in humans: muscle and systemic effects. If Physiol Lond 494: 891-898, 1996

6. Asp, S., and E. A. Richter. Decreased insulin action on muscle glucose transport after eccentric contractions in rats. J Appl Physjol 81: 1924-1928, 1996.

7. Carlson, B. M., and J. A. Faulkner. The regeneration of skeletal muscle libers following injury: A review. Med Sci Sports Exerc 15: 187-198, 1983.

8. Carter, G. T., N. Kikuchi, S. J. Horasek, and S. A. Walsh. The use of fluorescent dextrans as a marker of sarcolemmal injury. Histol Histopath 9: 443-447, 1994.

9. Clarkson, P. M., and I. Tremblay. Exercise-induced muscle damage, repair, and adaptations in humans. I Appl Physiol 65: 1-6, 1988 .

10. Costill "D. L., D. D. Pascoe, W. J. Fink " R. A. Fobergs, S. 1. Barr, and D. Pearson. Impaired muscle glycogen resynthesis atter eccentric exercise. I Appol Physiol 69: $46-50,1990$.

11. Curtin, N. A., and R. E. Davies. Chemical and mechanical change during stretching of actiwated frog skeletal muscle. Symposia on Quantitative Biology XXV1: 619-626, 1973.

12. Doyle, J. A., W. M. Sherman, and R. L. Strauss. Effects of eccentric and concentric exercise on muscle glycogen replenishment. I Appl Physiol 74: 1848-1855, 1993.

13. Duan, C, M. D. Delp, D. A. Hayes, P. D. Delp, and R. B. Armstromg. Rat skeletal muscle mitochondrial $\left[\mathrm{Ca}^{2+}\right]$ and injury from downhill walking. J Appl Physiol 68: $1241-1251,1990$.
14. Duan, $\mathrm{C}_{\mathrm{i}}$ and W. W. Winder Nenve stimulation decreases malonyl-CoA in skeleftal musclia. I App Physiol 72:901-904, 1992.

15. Dyck, D. ل, S. A. Peters, P. S. Wendling, A. Chesiey, E. Hultman, and L. L. Spriet. Regulation of glycogen phosphorylase activity during intense aerobic cycling with elevated FFA. Am J Physiol 265: E1 16-EN25, 1996.

16. Dyck, D. Ji, C. T. Putman, G. J. F. Heigenhauser, E. Huitman, and L. L. Spriet. Regulation of latcarbohydrate interaction in skeletal muscle during inlense aerobic cycling. Am J Physiol 265: E852-E859, 1903.

17. Ebbeling $C_{n}$. B., and P. M. Clarkson Exercise induced muscle damage and adaptation. Sports Med 7. $207-234,1989$

18. Evans W. J., and l. G. Cannon. The metabolic effecls of exercise-induced muscle damage. Exerc Sport Sci Rev 19: $99125,1991$.

19. Fenn, $W, O$. A quantithative comparison between the energy liberated and the work performed by the isolated sartorius muscle of the frog. I Physio/ 58: 175. $203,1923$.

20. Fluckey, J. D. M. S. Hickey, J. K. Brambrink, K. K. Hart, K. Alexander, and B. W. Craig. Effects of resistance exercise on glucose tolerance in normal and glucose-intolerant subjects. $J$ Appl Physiol 77: 1087-1092, 1994.

21. Franzini-Armstrong, $C$. The structure of a simple $Z$ line. J Cell Biol 58: 630 642, 1973.

22. Friden, $d$. Exercise-induced muscle soremess" a qualitative and quantitative sfudy of human muscle morphalogy and function. Umea University Medical Dissertations New Series, 1983.

23. Fridèrn, J., and P. L. Lieber. Structural and mechanical basis of exercise-induced muscle injury. Med Sci Sponts Exerc 24: 521-30, 1992

24. Fridên, J. R. L. Lieber, and L. E. Thomell. Subtlie indications of musclle dlamage lollowing eccentric contractions. Acta Physiol Scand 142: 523-4, 1991.

25. Helliwell, T. R, M. J. Jackson, J. Phoenix, P. MacLennan, J. J. West, and R. H. Edwards. Immunohistochemical and biochemical indicators of muscle damage in witro; the stability of control muscle and the effects of dinitrophenol and calcium ionophore. Int d Exp Pathai 75: 329-43, 1994. 
26. Huitman, $E_{i n}$ P. L. Greenhaff, J. M. Ren, and $K$. Sodertund. Energy metabotism and fatigue during intense muscle contraction. Regulation in melabolism. edited by P. H. Sugden, Londan, 1990.

27. Huxley, A. F. Muscle sinuclure and theories of contraction. Biophysics and Blophsyical Chemistry 7 . 2555318. 1957.

28. Jones, D. A, and J. M. Round. Skeletal muscle in health and disease, a textbook of muscle physjology. Manchester: Manchester Universily press, 1993.

29. Kinwan, J. P, R. C. Hickner, K. E Yarasheski, W. M. Kohrt, B. V. Wiethop, and J O. Holloszy. Eccentric exercise induces transient insulin resistance in healthy individuals. J Appl Physiol 72: 2197-202, 1992.

30. Kuipers, H. H. A. Keizer, F. T. J. H. Verstappen, and D. L. Costill. Influence of a prostaglandin-inhibiting drug on miscle soreness after eccentric work. Int $d$ Sports Med 6: 336-339, 1985.

31. Labeit, $S_{\text {., and }}$ : Kolmerer. The complete primary structure of human nebulin and its correclation to muscle structure. J Mol Biol 248: 308-315, 1995.

32. Lazarides, E. Intermediate filaments as mechanical integrators of cellular space. Nature 283: 249-256, 1980 .

33. Lleber, R. L., and J. Friden. Muscle damage is not a function of muscle force buit active muscle strain. J Appl Physiol 74: 520-6, 1993.

34. Lieber, R. L., L. E. Thornell, and J. Friden. Muscle cytoskeletal disruption occurs within the first 15 min of cyclic eccentric contraction. J Appl Physiol 80: 278-84, 1996.

35. Lieber, F. L., T. M. Woodburm, and J. Friden. Muscle damage induced by eccentric contractions of 25\% strain. J Appl Physiol 70: 2498.507, 1991.

36. Loeb, G. E., C. A. Pratt, C. M. Chanaud, and F. J. R. Richmond. Distribution and innervation of short, interdigitated muscle fibers in parllet-fibered muscles of cat hindlimb. J Morphol 191: 1.15, 1987 .

37. Mc.Cully, K. K., and J. A. Fautikner. Characteristics of lengthening contractions associated with injury to skeletal muscle fibers, J App! Physiol 61: 293-299, 1986.

38. McCully, K. K, and J. A. Faukner. Injury to skeletal muscle tibers of mice following lengthening contractions. IApp/ Physiol 59: 119-126, 1985.

39. Newham, D. J., D. A. Jones, and P. M. Clarkson. Repealed high-force eccentric exercise: effects on muscle pain and damage. J Appl Physio 63: $1381-6$ 1987

40. Newton, I. Philosophiae Naturalis Principia Mathematica. , 1687.

41. Nosaka, K, and P. M. Clarkson. Muscle damage following repeated bouts of high force eccentric exercise. Med Sci Sports Exerc 27: 1263-1269, 1995.

42. Nosaka, K., P. M. Clarkson, M. E. McGuiggin, and J. M. Byrne. Time course of muscle adaptation after high force eccentric exercise. Eur J App/ Physiol 63: 70-76, 1991.

43. O'Reilly, K. P. M. J. Warhol, R. A. Fielding, W. R. Frontera, C. N. Meredith ${ }_{y}$ and W. J. Evans. Eccentric exercise-induced muscle damage impairs muscle gllycogen repletion. J Appl Physiol 63: 252-256, 1987.

44. Odland , L. M., G. J. F. Heigenhauser, G. D. Lopaschuk, and L. L. Spriet. Humam skeletal muscle malonyl-COA at rest and during prolonged submaximal exercise. Am J Fhysiol 270: E541-E544 1906.

45. Ogilvie, R. W. R. B. Armstrong, K. E. Baird, and C. L Bottoms. Lesions in the ral soleus muscle following eccentrically biased exercise. Am J Anat 182: 335-346, 1985.

46. Patel, T. J, and P. L. Leiber. Force Transmission in Skeletal Muscle: from Actomyosin to External Tendons. In: Exercise and Spont Sciences Reviews, edited by J. O. Holloszy. St. Louis: Williams \& Wilkins, 1997, p. 321363.

47. Plato, P., W. M. Sheman, J. Betts, and J. M. Lash. Eccentric \& concentric exercise and insulin action. Med Sci Sports Exrea 21: S30. 1989

48. Prentki, $M$, and B. E. Corkey. Are the B-cell signalling molecules Malonyl-COA and cytosolic longchain acyl-COA implicated in multiple lissue defects of obesity and NIDDM? Qlabetes 45: 273-283, 1996.

49. Price, M. G., and E Lazarides. Expression of intermediate filament-associated proteins paranemin and synemin in chicken development. J Cell Biol 97 : 1860-1874, 1983.

50. Randle, P. J., E. A. Newsholme, and P. B. Garland. Regulation of glucose uptake by muscle. Effects of fatty acids, ketone bodies and pyruvate, and of alloxandiabetes and stanvation, on the uplake and metabolic fate of glucose in wat heari and diaphragm muscles. Biochem J93: 652-664, 1964. 
51. Sacco, P., and D. A. Jones. The protective effeet of damaging eccentric exercise. Second world congres of biomechanics, Amsterdam, p. 123, 1994.

52. Sacco, P., D. A. Jones, J. R. T. Dick, and G. Vrbova. Contractile properties and susceptibility to exerciseinduced damage of normal and $m d x$ mouse tibialis anterior muscle. Chin Sci 82: 227-36, 1992.

53. Saha, A. K., T. G. Kurowski, and N. B. Ruderman. A malonyl-CoA fuel sensing mechanism in muscle: effects of insulin, glucase and denervation. Am J Physiol 269: E283-E289, 1995.

54. Schwane, J. A, and R. B. Armstrong. Effect of trainirg on skeletal muscle injury from downhill running in rats. J'Appl Physiol 55: 969-75, 1983.

55. Sherman, W. M., J. M. Lash, J. C. Simonsen, and S. A. Blocmtield. Eftects of downhill running on the responses to an oral glucose challenge. Int J Sports Nutr2: $251-259,1992$

56. Smith $h_{x}$ L. L. Acute inflammation: the underlying mechanism in delayed onset muscle soreness? Med Sci Sponts Exerc 23: 542-551, 1991.

57. Stauber, W. T. Eccentric action of muscles: Physiology, injury, and adaptation. Exerc Sports Sci Rev 17: 157-185, 1989.

58. Stauber, W. T., P. M. Clarkson, V. K. Frilz, and W. J. Evans. Extracellular matrix disruption and pain atter eccentric muscle action. J App/ Phys 69: 868-874, 1990.

59. Stauber, W. T. V. K. Fritz, and K. K. Knack. Connective tissue response to muscle damage. Second world congress of biomechanics, Amsterdam, p. 122,1994

60. Stauber, W. T., G. R. Miller, J. G. Grimmet, and K. K. Knack. Adaptation of rat soleus muscles to 4 wh of intermititent strain. J Appl Physiol 77: 58-62, 1994.

51. Stevens, E. D. Effect of phase of stimulation on acute damage caused by eccentric contractions in mouse soleus muscle. I Appl Physiol BO: 1958-1962. 1996.

62. Street, S. F. Lateral transmission of tension in Irog myotibers: a myofibrillar network and transwerse cytoskeletal connections are possible transmitters. J Cell Physid 1 14: 346-364, 1983.

63. Takala, T. E. S., S. O. A. Koskinen, J. Komulainen, M. K. C. Hesselink, V. Vinko, H. Kuipers, and W. Kowanen. Eccentric contractions increase expression of fibrillar collagens, prolyl 4-hyroxylase and lysyl oxidase in skeletal muscle. Med Sel Sports Exerc 28: $\$ 153,1996$.

64. Trinick, J. Titin and nebulin protain rulers in muscle? Trends Biochem Sci 19: 405-409, 1994.

65. wan dep Meulen, J. H. H. Kuipers, and J. Drukker. Relationship between exercise-induced muscle damage and enzyme release in rats. J Appl Phys 71: $999-1004,1991$.

66. van der Meulen, J. H., H. Kuipers, F. F. M. Stassen H. A. Keizer, and G. J. van der Vusse. High energy phosphales and related compounds, glycogen levels and histology in the rat libialis anterior muscle after forced lengthening and isometric exercise. Pflügers Arch 420: 354-358, 1992

67. Walliman, T., M. Wyss, D. Brdiczka, K. Nicolay, and H. M. Eppenberger. Intracellular compartmentation. structure and function of creatine kinase isoenzymes in tissues in tissues with high and fluctuating energy demands" the "phosphocreatine circuit" for cellular energy homeostasis. Biochem d 281: 21-40, 1992.

68. Wang, K. R. , A. McCarter, J. Wright, J. Beverly, and R. Ramirez-Mitchell. Viscoelasticity of the sarcomere matrix of skeletal muscles: The titin-myosin composite thament is a dual-slage molecular spring. Biophys d 64: 1161-1177, 1993

69. Warren, G. L. D. A. Hayes, D. A. Lowe, B. M. Prior, and R. B. Armstrong. Materials fatigue initiates eccentric contraction-induced injury in rat soleus muscle. J Physiol 464: 477-480, 1993.

70. Warren, G. L., D. A. Lowe, D. A. Hayes, C.J. Karwoski, B. M. Prior, and P. B. A.rmsitrong. Excitalion lailure in eccentric conlraclion-induced injury of mouse saleus muscle. J Physiol London 468: $487 \times 499$, 1900.

71. Widrick, J. A, D. L. Costill, G. K. Mc.Conell, D. E. Anderson, D. F. Pearson, and d. J Zachwieja. Time course of glycogen accumulation afler eccentric exercise. J Appl Physiol 72: 1999-2004 "1992.

72. Winder, W. W., J. Arogyasami, R. J. Barton, I. M. Elayan, and P. Ri. Vehrs. Muscle malonyl-CoA decreases during exercise, J Appl Phsyiol 67: 22302233. 1989. 

Characterization and Testing of the Experimental Set-Up and Exercise Protocols Used 


\section{Introduction}

Skeletal muscle function relies on both muscle architecture and intrinsic properties of muscle fibers, e.g., fiber typing. Skeletal muscle performance has been tested under wide variety of experimental conditions, ranging from studying single muscle fibers in vifro to non-invasive models where the muscle(group) of interest is studied in the intact animal. Muscle function in animal models can be accurately measured by dissecting the muscle from the surrounding tissue and attaching the distal tendon with a small section of bone to a lever arm $(4,6)$, or to cut the exposed distal tendon and fix it to the lever arm of a servo motor (8). As a consequence of the surgery these types of models primarily permit acute experiments. To monitor long-term effects of (multiple) intervention(s), measurements on intact muscle tendon complexes with minimal surgical intervention are preferred.

In the present thesis two different experimental set-ups have been used. The experimental set-up used in the studies outlined in chapter 3 and 4 made it possible to induce shortening, lengthening and isometric muscle contractions of dorsi-and plantarflexion of the tibiotarsal joint in intact rats. However, muscle force as a parameter of muscle function could only be monitored post-exercise. From a functional perspective the quantity of work performed during muscle contraction is obviously as important as post-exercise muscle force. Therefore a new AshtonMiller like (1) experimental set-up (named rat dynamometer) was constructed to non-invasively measure work of rat plantar-and dorsiflexor hindlimb muscles during contraction under strictly standardized conditions. This rat dynamometer (RDM) has been extensively tested and validated before using it for the studies described in chapter 5,7 and 8 .

In the present chapter we will provide a detailed technicall description of the rat dynamometer. Furthermare, we will characterize different exercise protocols and outline the effects of these protocols on external work during muscle contraction.

\section{Materials and methods}

\section{Description and validation of the experimental set-up}

The experimental set-up consists of 4 main units (Figure 2.1), a custom built rat dynamometer (ADM), a control unit to adjust RDM settings (e.g., rotational velocity and range of motion) as desired, an Apple Macintosh 7100 PowerPC ${ }^{B}$ with an 8 channel, 12 bits Lab-NB analog-to-digital conversion board (National Instruments $\left.{ }^{(}\right)$programmed with LabVIEW 3.1 and a pulse generator for purpose of electrical stimulation and triggering of the RDM (HSE 215/IZ, Freiburg, Germany).

The RDM consists of a platform with 6 degrees of freedom, an adjustable femur fixation, a rotational footplate, a linear motor and a potentiometer. An anaesthetized rat is placed in supine position on the heated $\left(37^{\circ} \mathrm{C}\right)$ platform. After positioning the rat in the horizontal plane, the foot is secured to the footplate with two-sided adhesive tape while the instep is covered with a narrow fitting thin aluminum plate. The femur is fixed distally with a hinge fixation at a $\sim 90^{\circ}$ flexion in the knee. To induce rotation in the ankle joint, the footplate is secured to the axis of the linear inotor. 


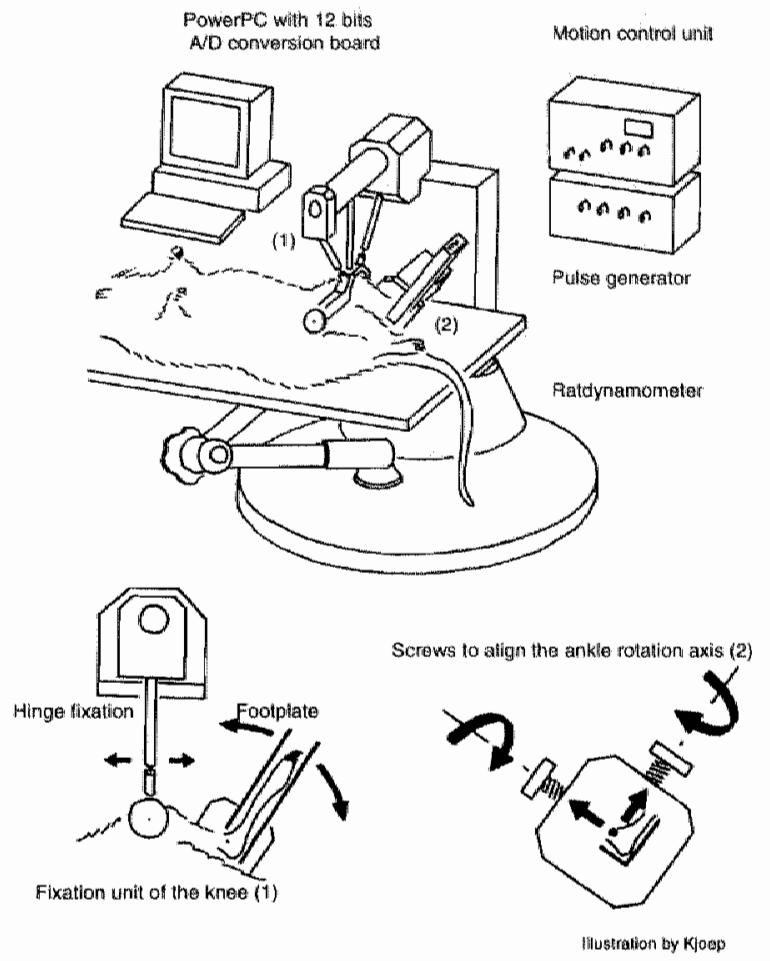

Figure 2.1: Overview of the experimental set-up. The upper panel shows a rat in the RDM and the 4 main units of the set-up used (i.e., a computer with A/D board, the RDM, a unit to adjust settings of the RDM, and a pulse generator). The lower left panel shows the fixation unit of the knee in more detail and the way the foot is secured to the mobile unit. The lower right panel indicates how the rolation axis of the ankle can be aligned with the rotation axis of the mobile unit.

Testing of the dorsiflexor as well as the plantarflexor muscle group of one leg was possible by either clockwise or counter clockwise rotation. The femur fixation unit could be placed at both sides of the axis and the footplate could be rotated in the vertical plane to face the femur fixation unit. As a consequence, both hindlimbs can be tested.

Fixation of the femur to prevent lateral movement of the knee is achieved by two conical pieces placed on the skin between the femoral and tibial condyles. To prevent force transmission through the tibia towards the femur fixation unit, the femur fixation unit was connected to the body of the RDM by a hinge fixation, enabling small movements parallel to the tibia. The ankle rotation axis was aligned with the rotation axis of the RDM by translation of the footplate relative to the rotation axis of the RDM over $2 \mathrm{~mm}$ in 2 perpendicular directions. Malalignment of the ankle axis causes visible movement of the femur fixation unit which can be reduced by translation of the footplate. 
The footplate is rotated using a linear motor with an adjustable isokinetic velocity between $1 . \mathrm{s}^{-1}$ and $1000^{\circ} \mathrm{s}^{-1}$. Both dorsal and plantarflexion can be performed over linear range of $-40^{\circ}$ to $+40^{\circ}$ with respect to the anatomical position (ankle angle $90^{\circ}$ ). Axis angle, representing the actual ankle angle, is monitored with a precision potentiometer (alinearity $<0.5 \%$, full scale $10 \mathrm{~V} ; 1 \mathrm{~V}$ equals $10^{\circ}$ ).

Total torque is assessed from the voltage through the linear motor of the RDM (full scale $10 \mathrm{~V} ; 1 \mathrm{~V}$ equals $30 \mathrm{mNm}$ ). Total torque equals the sum of frictional torque, inertial torque, muscle passive torque and, if any, muscle active torque. To calculate active torque, torque of a passive stroke (i.e., to a rotation without muscle contraction) was subtracted from torque measured during muscle contraction (active stroke).

Rotation of the RDM was gated by analog triggering of the control unit of the RDM. Analog output signals from the RDM were digitized on-line. Three channels (12 bits, scan rate $1000 \mathrm{~Hz}$ ) were used for data collection. One channel received the urigger signal, while the other two received data from RDM, either from the linear motor, representing total torque, or from the patentiometer, representing the actual angle of the axis.

External work of the active muscle group is calculated on-line as the integration of active torque over rotation angle using the following formula:

$$
W=\sum_{i=1}^{n-1} \frac{T(i)+T(i+1)}{2} \times\{\phi(i+1)-\phi(i)\}
$$

Where $W$ represents external work $(J), T$ represents torque $(\mathrm{Nm})$ and $\phi$ represents ankle angle (rad).

The accuracy of assessment of external work by the experimental set-up was verified by testing a torsion spring in the RDM and comparing work computed by the experimental set-up with predicted work based on the calibration curve of the torsion spring. The torsion spring was calibrated by balancing the spring with precision weights over the entire range of motion of the RDM and fitting a line through the obtained data. Predicted work of the torsion spring was then calculated as the area under the calibration curve over the same range used while testing the torsion spring in the RDM.

Variation in external work was monitored at rotational velocities of 50,100 , 250 and $500^{\circ} \cdot \mathrm{s}^{-1}$ under three different conditions: unloaded strokes (without a rat in the apparatus, to test variation in inertial and frictional work), using the torsion spring and during a passive stroke (with a rat in the apparatus but without stimulation of the dorsiffexor muscles). In total 60 rotations were recorded under all testing conditions. Furthermore, external work during 2400 unloaded strokes with a rotational velocity of $500^{\circ} \cdot \mathrm{s}^{-1}$ was recorded.

During active strokes (with stimulation of the dorsiflexor muscles) the RDM was triggered at the onset of electrical nerve stimulation. To enable build up of muscle tension, an adjustable delay was maintained between stimulation of the nerve and the onset of rotation in the ankle joint. Since there was no rotation during this quasi-isometric pre-contraction, work was not biased by this pre-contraction. Promptly after reaching the adjusted ankle angle, stimulation of the nerve was 
terminated. The passive isokinetic backstroke was performed with a rotational velocity of $100^{\circ} \cdot \mathrm{s}^{-1}$ and was initiated $100 \mathrm{~ms}$ after reaching the adjusted ankle angle.

\section{Electrodes used for electrical stimulation}

Two types of electrodes have been used. Percutaneous needle electrodes for stimulation during repeated measurements and wire electrodes for stimulation on the exposed nerve during acute experiments. Electrode positioning and exercise were performed under pentobarbital anesthesia (Nembutal $1.0 \mathrm{ml} / \mathrm{kg}$ body weight, i.p. injection) after short term ( $<20$ s) $\mathrm{CO}_{2}$ sedation. For positioning of both types of electrodes the skin was depilated just distal of the caput fibulae at the lateral side of the hindlimb.

If using percutaneous needle electrodes, the negative pole of the needle was positioned distal of the caput fibulae through the transitional area of the lateral gastrocnemius and the tibialis anterior muscle close to the common peroneal nerve. The positive pole of the needle electrode was placed percutaneous, distal to the cathode at the ventral side of the tibialis anterior muscle. When a voltage over $4 \mathrm{~V}$ was needed to obtain maximal muscle contraction, the cathode was repositioned to stimulate closer to the common peroneal nerve. To prevent electrode displacement due to rotation, muscle contraction or a combination of the two, both needle electrodes were fixed using cyanoacrylate glue when positioning was optimal (maximal work with voltage $<4 \mathrm{~V}$ ).

In case of stimulation at the exposed nerve, a small $(5 \mathrm{~mm})$ incision was made through the before mentioned transitional area and the negative pole of a flexible stainless steel wire electrode was carefully hooked to the common peroneal nerve. One or 2 stitches were used to close the incision. The positive electrode was placed similar to the positive pole of the needle electrodes.

\section{Exercise protocols}

In the present thesis two exhaustive exercise protocols (one shortening and one lengthening) have been used. During both exhaustive exercise protocols the rat hindlimb dorsiflexor muscles (TA and EDL) performed 4 consecutive exercise bouts of 60 isokinetic $\left(500^{\circ} \cdot \mathrm{s}^{-1}\right)$ fused tetanic contractions. Muscles were stimulated via the exposed common peroneal nerve every $3000 \mathrm{~ms}$ for $310 \mathrm{~ms}$ $(\sim 3 \mathrm{~V}, \sim 130 \mathrm{~Hz})$, subsequent exercise bouts were interspaced by a 5 minute recovery period. Rotation ( $50^{\circ}$ rotation in ankle angle) of the RDM was initiated 210 ms after the onset of nerve stimulation. Work was recorded every single contraction, total work was computed as the sum of work during all 60 contractions in one exercise bout. Counter clockwise rotation induced shortening contractions while clockwise rotation induced lengthening contractions.

Inter-subject variation in muscle work was studied under both lengthening and shortening conditions ( $\mathrm{n}=36$ in both groups). Hindlimb dorsiflexor muscles performed 4 sessions of 60 shortening or lengthening contractions. Preceding all exercise bouts, muscle passive torque was recorded after 3 passive strokes. Subsequently, active muscle torque was calculated by subtracting passive torque from active torque during all successive active strokes. Every exercise bout ended with 2 passive strokes to monitor alterations muscle passive torque and in frictional and/or inertial torque during the exercise session. 
Intra-subject variation in contractile work was studied by performing repeated measurements in 4 rats in 2 exercise sessions of 4 consecutive exercise bouts of 60 shortening contractions. The two exercise sessions were interspaced by a 4 d recovery period to permit complete metabolic recovery.

Here we will also discuss 2 endurance like protocols. One of the endurance protocols was performed with a fixed stimulation frequency $(60 \mathrm{~Hz}$ ) (FF protocol) while in the other endurance protocol external work was fixed at a load of $-20 \%$ of the supramaximal recorded work (FW protocol) by continuously manipulating the stimulation frequency. Supramaximall work was assessed by steadily increasing the stimulation voltage (one stimulation every $15 \mathrm{~s}$ ) until external work plateaued while keeping the stimulation frequency at $150 \mathrm{~Hz}$ (known to induce fused tetani). Using the voltage leading to maximal muscle contraction the stimulation frequency was set at $20 \mathrm{~Hz}$ and steadily increased until external work equaled $-20 \%$ of the previously recorded maximal value. When external work started to deviate from $20 \%$ of the maximal value, the stimulation frequency was manipulated as required in order to maintain external work at the $20 \%$ level. Both endurance protocols had a tour-duty cycle of $10 \%$ (150 ms stimulation every $1500 \mathrm{~ms}$ ), lasted for 1 hour and comprised of 2400 shortening isokinetic contractions $\left(500^{\circ} \cdot \mathrm{s}^{-1}\right)$ over a $50^{\circ}$ ankle angle range.

To study the glycogen usage during both endurance protocols, muscle glycogen content was measured following 100, 350 and 2400 contractions and compared to values in the contralateral control leg. Muscle glycogen content was assessed by dissolving muscle tissue for 1 th in $1 \mathrm{M} \mathrm{NaOH}\left(37^{\circ} \mathrm{C}\right)$ and precipitating glycogen with $96 \%$ ethanal $(10 \mathrm{~min}$ at $80 \% \mathrm{C}$ followed by overnight precipitation at $4^{\circ} \mathrm{C}$ ). Subsequently the pellet was hydrolyzed using $1 \mathrm{M} \mathrm{HCl}$ at $100^{\circ} \mathrm{C}$ for $3 \mathrm{~h}$ after which $\mathrm{HCl}$ was neutralized with a $\mathrm{KCl}$ saturated $\mathrm{KOH} / \mathrm{Tris}(2.1 \mathrm{M} / 0.12 \mathrm{M})$ buffer. Glycogen derived glycosyl units were determined using a glucose kit (hexokinase method, Roche) for a centrifugal analyzer (Cobas Fara. Hoffman La-Roche, Switzerland) and expressed as $\mu \mathrm{mol}$ glycosyl units.g $\mathrm{g}^{-1} \mathrm{WW}$.

Since intense exercise may induce a fluid shift towards the exercised muscles and consequently affect muscle mass, it was chosen to assess muscle mass of the non-exercised leg. We previously observed no differences between muscle mass of dorsiflexor muscles of right and left legs in control rats. To estimate muscle mass without muscle dissection, the relation between body mass and muscle mass was assessed in 76 rats.

Fiber strain velocity measurement

The relationship between ankle angular velocity and muscle fiber strain velocity was assessed by recording the movement of fluorescent spheres attached to the muscie surface with 2 video cameras (3). The skin and epimysium covering the TA were carefully removed and 4 fluorescent polystyrene spheres $(\varnothing=0.7 \mathrm{~mm})$ were attached in a straight line, midsagittally on a muscle fascicle. Using a binocular microscope (20x magnification), the two proximal spheres were attached on the non-contractile part of the muscle (the proximal aponeurosis) while the two distal spheres were placed on contractile muscle tissue. The exposed muscle was intermittently moistured with saline. All spheres were Hlluminated according to van
Bavel et al. (9). 
Fiber strain velocities of TA muscle were calculated under passive and active conditions at 4 rotational velocities $\left(50^{\circ} \cdot \mathrm{s}^{-1}, 100^{\circ} \cdot \mathrm{s}^{-1}, 250^{\circ} \cdot \mathrm{s}^{-1}\right.$ and $500^{\circ} \cdot \mathrm{s}^{-}$ ${ }^{1}$ ) in one rat. Contraction was induced by electrical stimulation at the exposed common peroneal nerve during the entire range of motion $\left(50^{\circ}\right)$. Stimulation preceded the onset of rotation by $210 \mathrm{~ms}$, therefore the stimulus duration during successive rotational velocities equaled $1210,710,410$ and $310 \mathrm{~ms}$, respectively. Four active and 4 passive strokes were recorded at every velocity. Under active conditions 3 strokes at every velocity were completely elaborated, while under passive conditions 2 strokes per velocity were studied.

Synchronized video recordings were made from a ventral and a lateral view, to test pure sagittal movement and to measure strains. Frames from both video cameras (Adimec MX-5) were digitized on-line by a framegrabber (Magic RGB, $512 * 512$ pixels, $25 \mathrm{~Hz}, 8$ bits, Matrox). The integration time was set at approximately $4 \mathrm{~ms}$ per field using a custom-built timer. Centroids of all spheres were calculated after thresholding. Calculation of fiber strain velocity at $500^{\circ} \cdot \mathrm{s}^{-1}$ was performed after increasing temporal resolution to $50 \mathrm{~Hz}$ by separating odd and even fields. Since 2 spheres were attached on the aponeurosis and 2 at the muscle, engineering strain could be computed at 3 segments, one at the aponeurosis, one at the muscle and one on a non-specified transitional area. Engineering strain of the muscle segment was calculated relative to the nonstimulated initial length using the following formula:

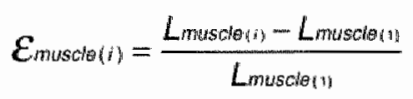

Where $\varepsilon$ stands for strain in the muscle segment, Lmuscien (n stands for segment length when the first image was recorded and ankle angle was still at the starting position, while $L_{\text {muscle(i) }}$ represents segment length in image $i$. Fiber strain velocity was calculated by dividing the calculated change in strain by the time span between consecutive images (typically $40 \mathrm{~ms}, 20 \mathrm{~ms}$ after separation of odd and even fields).

\section{Statistics}

For assessment of the calibration curve of the torsion spring a second order polynome (independent variable axis angle (rad), dependent variable torque of torsion spring) was fitted through the obtained data. Variation of external work was assessed using descriptive statistics (mean, standard deviation and variance) and a one-way ANOVA was performed to study differences in successive rotational velocities. Sheffé post-hoc analyses were done to localize differences, if existing. Inter-subject variation was monitored using descriptive statistics (mean and standard deviation). Paired Students $t$-tests were used to test intra-subject differences. Significance was accepted when p-values were below 0.05 . Linear regression analysis was used to assess the relationship between body mass and muscle mass as well as to assess the relation between ankle angular velocity and fiber strain velocity. All statistical analysis were performed using the StatView ${ }^{(B)}$ 4.02 statistical package. 


\section{Results}

Description and validation of the experimental set-up

The correlation coefficient of the fitted calibration curve of the torsion spring equaled 0.99. External work of the torsion spring measured by the RDM was independent of the rotational velocity (Table 2.1) had a low variation (variance did not exceed $0.053 \mathrm{~m} J)$. Mean external work of the torsion spring measured by the RDM $(37.3 \mathrm{~mJ})$ was on average $3 \%$ higher than predicted work based on calculation $(36.2 \mathrm{~mJ})$.

\begin{tabular}{|c|c|c|c|c|}
\hline & & Torsion spring & Unloaded strokes & Passive strokes \\
\hline \multicolumn{5}{|l|}{$50 \% / s$} \\
\hline & Mean & 37.22 & 0.324 & 0.393 \\
\hline & Std. Dev. & 0.194 & 0.148 & 0.166 \\
\hline & Vairiance & 0.038 & 0.022 & 0.028 \\
\hline \multicolumn{5}{|l|}{$100^{\circ} / \mathrm{s}$} \\
\hline & Mean & 37.74 & 0.122 & 0.136 \\
\hline & Std. Dev. & 0.153 & 0.064 & 0.074 \\
\hline & Variance & 0.023 & 0.004 & 0.006 \\
\hline \multicolumn{5}{|l|}{$250 \% / \mathrm{s}$} \\
\hline & Mean & 37.19 & 0.145 & 0.271 \\
\hline & Std. Dev. & 0.231 & 0.091 & 0.155 \\
\hline & Variance & 0.053 & 0.008 & 0.024 \\
\hline \multicolumn{5}{|l|}{$500 \% / 5$} \\
\hline & Mean & 37.33 & 0.126 & 0.157 \\
\hline & Std. Dev. & 0.092 & 0.054 & 0.049 \\
\hline & Variance & 0.009 & 0.003 & 0.002 \\
\hline
\end{tabular}

Table 2.1: Mean, standard deviation and variance of work (m) recorded at 4 different rotational velocities during 60 shortening strokes with the torsion spring, without a rat (unloaded strokes) and with a rat under resting conditions (passive strokes).

Average external work during 60 unloaded or passive strokes stayed below $0.4 \mathrm{~mJ}$, with a low variance (Table 2.1). During 2400 strokes, passive external work gradually increased upto $0.8 \mathrm{~mJ}$ and averaged $0.44 \mathrm{~mJ}( \pm 0.16)$.

\section{Electrodes used for electrical stimulation}

Contractile work assessed promptly after electrode introduction was similar using either needle or wire electrodes. However, we observed that $24 \mathrm{~h}$ following stimulation at the exposed nerve using wire electrodes, contractile work declined with more than $50 \%$ in 10 out of 12 rats, in the other 2 rats, contractile work did not differ between successive exercise sessions. Contractile work during repeated measurements in 4 rats using percutaneous needle electrodes, remained unchanged.

Exercise protocols

Contractile work during every shortening or lengthening contraction is depicted in Fig 2.2 (upper panel). For sake of clarity, standard deviations are only depicted every $10^{\text {th }}$ contraction. Total work during successive exercise bouts of both shortening and lengthening exercise progressively declined (Fig 2.2, lower panel). 


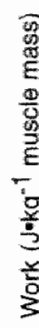

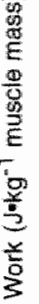

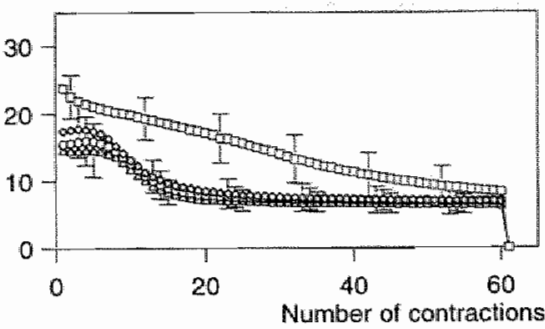

Number of contractions

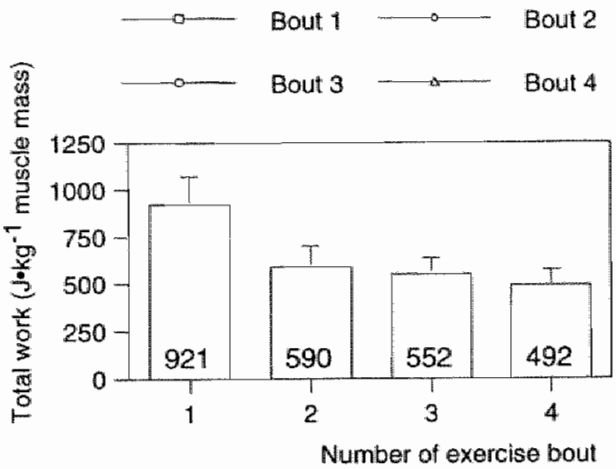

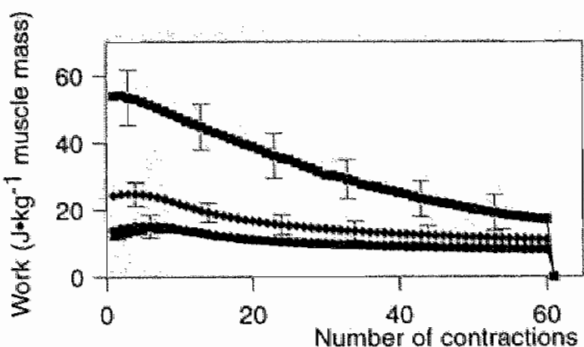

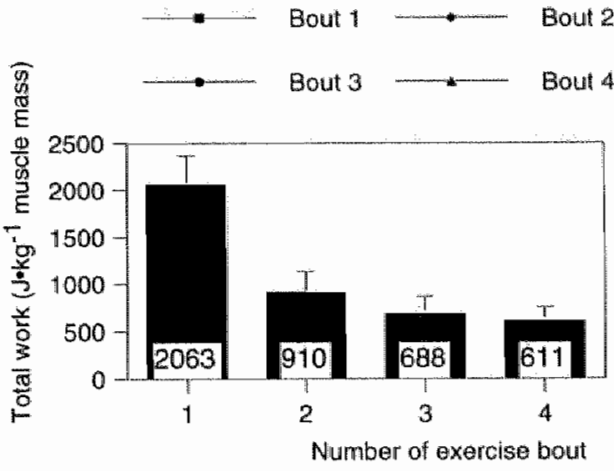

Figure 2.2: Upper left and right panel shows contractile work during successive shortening $(n=36)$ and lengthening ( $n=36$ ) contractions, respectively (data presented as mean with SD). The lower panel shows total work (computed as the sum of work during individual contractions) performed during the entire shortening (left panel) or lengthening (right panel) exercise session.

Mean contractile work $(n=4)$ recorded during the first exercise session never differed signifcantly from work recorded 4 days later in the same four rats. Total work during the first exercise session did not differ significantly from work during the second exercise session 4 d afterwards (Fig 2.3).
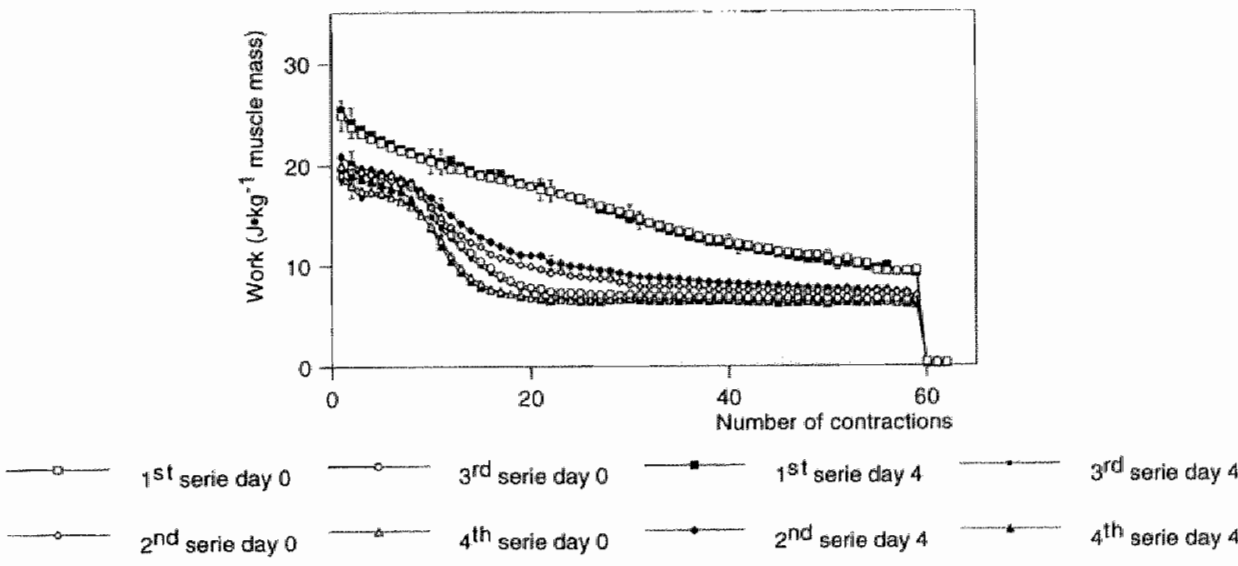


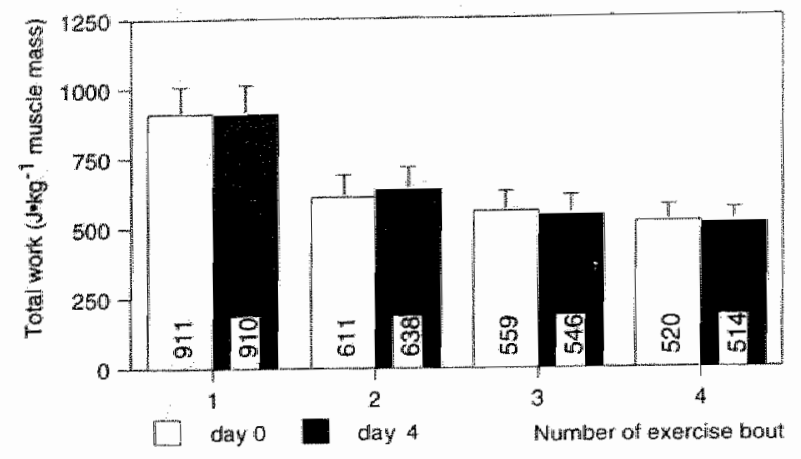

Figure 2.3: Upper panel shows average work during successive contractions under exhaustive shortening exercise conditions. Open symbols refer to the first exercise session, filled symbols refer to the exercise session 4 days later (data presented as mean with SD). The lower panel shows total work performed during the entire exercise session. No differences were observed between exercise on day 0 and day 4 .

Contractile work during both endurance protocols is shown in Fig 2.4. During the fixed frequency protocol (Fig 2.4, left panel) work rapidly dropped during the first 300 contractions and stabilized at $-20 \%$ of previously determined maximal work. During the fixed work protocol (Fig 2.4 right panel), work was stabilized by manipulating the stimulation frequency. At the onset of the fixed work protocol the stimulation frequency averaged $25 \mathrm{~Hz}$ and a steady increase upto $\sim 50 \mathrm{~Hz}$ was required to maintain external work at the $20 \%$ level.
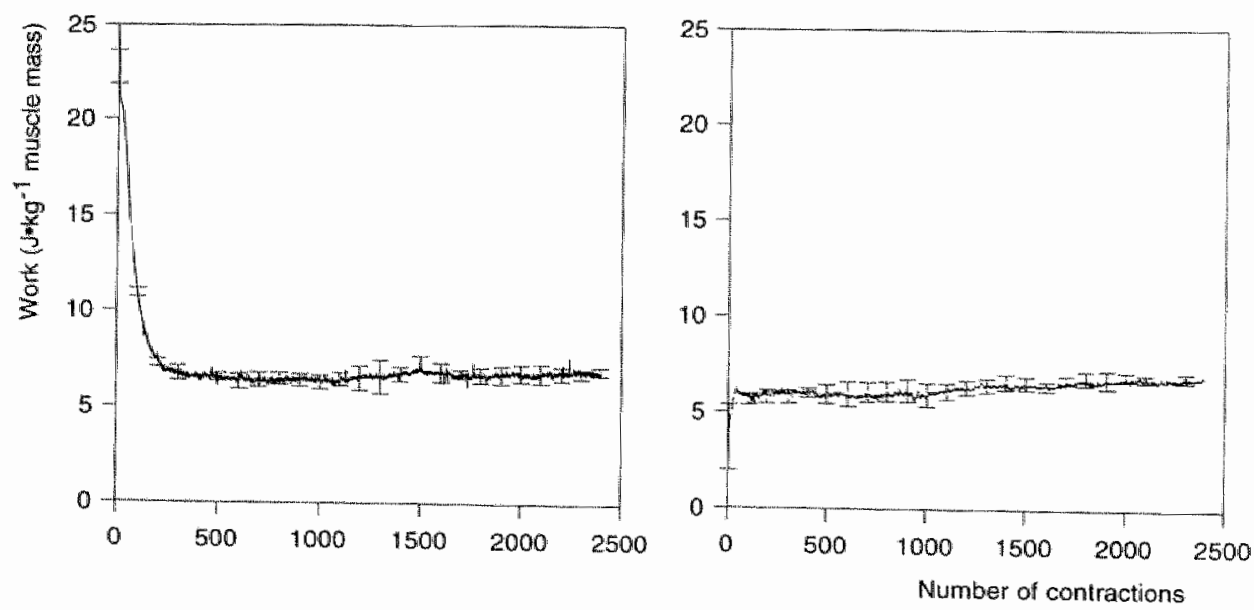

Figure 2.4: External work recorded during the fixed frequency protocol (left panel) and during the fixed work protocol (right panel, data presented as mean with $\mathrm{SD}, n=4$ ). 
Muscle glycogen content following 100,350 and 2400 contractions, as well as in the contralateral non-exercised leg is shown in table 2.2 for both protocols. Since muscle glycogen was only assessed in 4 rats at every sample point statistic analysis was omitted.

\begin{tabular}{lccc}
\hline & Fixed frequency $(60 \mathrm{~Hz})$ & Fixed work (20\% of maximal work) \\
\cline { 2 - 2 } Pre-exercise & $164.6(12.9)$ & $165.3(17.7)$ \\
100 contractions & $53.7(3.9)$ & $68.9(12.9)$ \\
350 contractions & $50.1(4.3)$ & $60.2(14.1)$ \\
2400 contractions & $39.1(2.5)$ & $32.2(6.1)$ \\
\hline
\end{tabular}

Table 2.2: Muscle glycogen content ( $\mu$ mol. $\mathrm{g}^{-1}$ ww) during fixed frequency and fixed work protocol, preexercise values as well as glycogen content after 100,350 and 2400 contractions are presented. Preexercise values are assessed in contralateral non-exercised control muscle. Data are presented as mean $( \pm S D, n=4)$.

Regression analysis of muscle mass in relation to body mass showed a linear relationship (Fig 2.5).

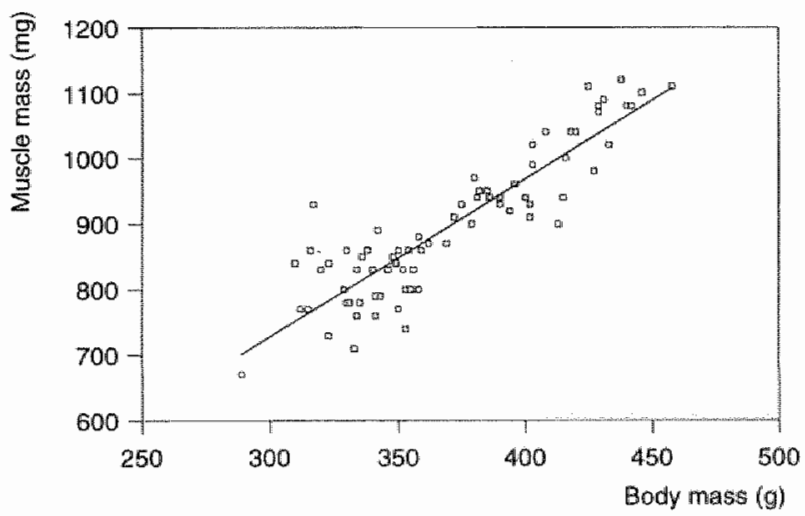

Figure 2.5: Linear regression plot of body mass vs. muscle mass, giving rise to the following regression equation: Muscle mass $(\mathrm{mg})=2.4221 \cdot$ body mass $(g)\left(n=76, R^{2}=0.803\right)$

\section{Fiber strain velocity measurement}

Video recordings from the ventral view showed that during rotation all spheres stayed in one line and that segments showed a rotation $<5^{\circ}$, introducing fictuous strains of less than $0.5 \%$. Therefore, anly data of the lateral view will be discussed here. During passive rotation from $-20-30^{\circ}$, being the range of rotation used in all studies in the present thesis, TA muscles were lengthened $\sim 10 \%$. During the fixedend precontraction, the aponeurosis segment showed an average lengthening of $16 \%$ while average muscle shortening during the fixed-end precontraction equaled $18 \%$ (Fig 2.6). Aponeurosis strain remained constant during rotation while muscle fiber strain increased during ankle rotation. Linear curve fitting was performed to 
describe the relationship between fiber strain velocity and ankle velocity, resulting in the following two equations:

$$
\begin{aligned}
& F S V_{p}=1.774 \cdot 10^{-3} T T V\left(R^{2}=0.999\right) \\
& F S V_{a}=2.822 \cdot 10^{-3} T T V\left(R^{2}=0.979\right)
\end{aligned}
$$

Where $F S V_{p}$ and $F S V_{a}$ represents passive and active fiber strain velocity $\left(L_{f} \cdot S^{-1}\right)$, respectively and TTV stands for ankle velocity $\left({ }^{\circ} \cdot \mathrm{s}^{-1}\right)$.

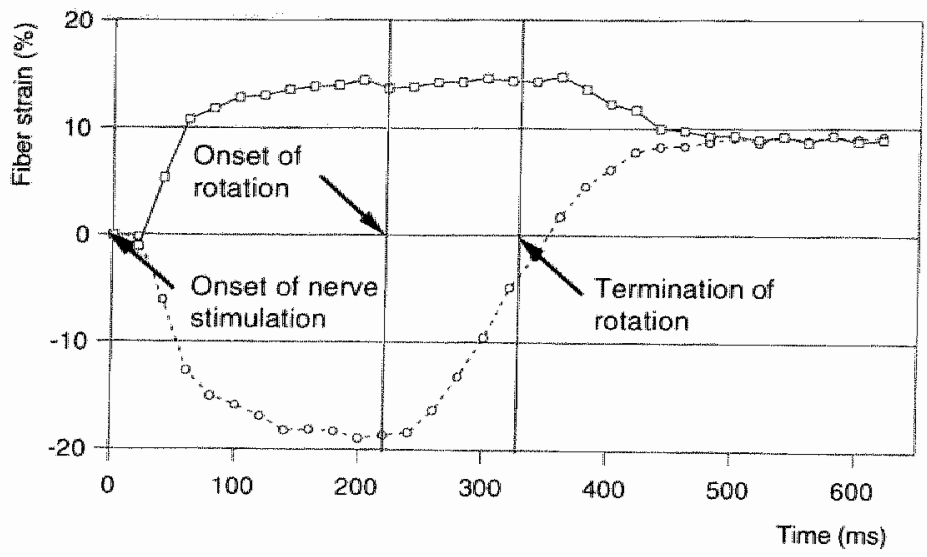

Figure 2.6: Typical profile of strain in the aponeurosis segment (solid line with squares) and the contractile segment (dotted line with circles) studied during a fused tetanic lengthening contraction $\left(500^{\circ} \cdot \mathrm{s}^{-1}\right)$. Note lengthening of the aponeurosis segment and simultaneous shortening (of the same order of magnitude) of the contractile segment during the $210 \mathrm{~ms}$ 'isometric' pre-contraction. During lengthening no alterations in aponeurosis segment length were observed.

\section{Discussion}

Muscle contractile performance can be assessed in different experimental set-ups ranging from in vitro measurements on single muscle fibers $(5,10)$ to in vivo measurements in intact animals $(1,2,7)$. From a functional viewpoint we favored to monitor work during contraction. For sake of monitoring contractile work in intact animals, a dynamometer for rat hindlimb dorsi-and plantarflexor muscles was developed and tested. In the rat dynamometer (RDM) described here, muscle contractile velocity is adjusted by manipulation of ankle rolational velocity. Since muscle fiber strain velocity is of more importance than ankle rotational velocity when studying muscle function, we attempted to relate ankle rotational velocity to muscle fiber strain velocity.

Description and validation of the experimental set-up

Muscle contractile work was assessed by calculating the area under the ankle angle-torque curve. Torque was measured by determining the voltage through the linear motor of the RDM. Measuring torque in the rotational axis as close to the footplate as possible is preferable to minimize inertial effects. However, this would imply an ill-favored loss of stiffness. The sum of inertial and frictional work during rotation was measured by recording a passive stroke previous to every subsequent 
active stroke. Every subsequent stroke was corrected for inertial and frictional work by subtracting work during a passive stroke from work during an active stroke. Since passive strokes were recorded only once (previous to all subsequent active strokes) it is crucial that both inertial as well as frictional torque remain stable (1.e.. are very reproducible) during the entire exercise period. Testing this by recording 60 unlloaded (without a rat) and 60 passive (with a rat) strokes in the RDM revealed that the sum of inertial and frictional work during passive strokes did not exceed $0.40 \mathrm{~mJ}$ and had a low variance $(<0.028$ ), passive work during 2400 rotations

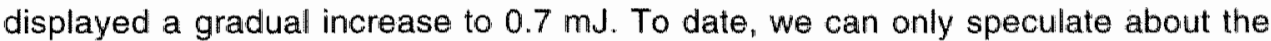
origin of the increase in passive work. It could be reasoned that minor changes in the way the rat is positioned in the set-up are responsible for the increase in passive work. However, we have no experimental data supporting this hypothesis.

Work measured by the RDM during rotation of a calibrated torsion spring was on average $3 \%$ higher than work computed using the calibration curve of the torsion spring. This deviation was a highly reproducible and systemic deviation, therefore, work measured by the RDM appears to be an accurate and valid reflection of actual work. Since muscle contractile work is determined identical to the way work of the torsion spring was measured, contractile work determined by the RDM is a valid and accurate parameter for muscle contractile performance.

Electrodes used for electrical stimulation

Direct stimulation at the exposed nerve, using wire electrodes, has the advantage that the site of contact with the nerve can be visually checked. Furthermore, displacement of the electrode during exercise is rarely observed. Positioning of the electrode, however, can only be performed after minor surgery. A major advantage of percutaneous needlle electrodes for muscle stimulation, is its non-invasive character. Above all, the use of percutaneous needle electrodes for stimulation makes it possible to perform repeated measurements on consecutive days within the same rats. In case of repeated measurements of contractile work within the same rats, percutaneous stimullation with needle electrodes displayed reproducible work whereas direct stimulation at the exposed nerve using wire electrodes failed to do so.

When using percutaneous needle ellectrodes to induce muscle contraction it is crucial to optimize electrode positioning in order to minimize the stimulation voltage and consequently avoid antagonist muscle activity. Contractile work was assessed by steadily increasing the stimulation voltage until a plateau in work was reached. Further increment of the stimulation voltage gave lower work walues, indicating antagonist muscle activity. Therefore, the stimulation voltage of choice was kept as low as possible. Usually 4-5 contractions were needed to determine the stimulation voltage of choice.

\section{Exercise protocols}

Inter-subject variability in contractile work, as indicated by a low standard deviation, was somewhat lower during shortening exercise than during lengthening exercise. Since lengthening contractions induce muscle damage and consequently contractile malfunction, part of the higher variation during lengthening exercise may originate from inter-subject variations in susceptibility to muscle damage. To ensure that contractile work during exercise bouts indeed reflects active muscle 
work instead of changes in frictional or passive work, the final two strokes were recorded without nerve stimulation. On the average, work during the final 2 strokes remained below $0.5 \mathrm{~mJ}$. The profile of contractile work during the first exercise session and the one $4 \mathrm{~d}$ later was very much alike and no statistical differences were detected. Although work within one exercise session declined from $\sim 25 \mathrm{~J} \cdot \mathrm{kg}^{-}$ 1 muscle mass $(\mathrm{mm})$ during the first contraction to $-8 \mathrm{~J}^{\mathrm{kg}} \mathrm{k}^{-1} \mathrm{~mm}$ during the final contraction, contractile work was completely restored within $4 \mathrm{~d}$. This implicates that metabolic aberrations, if any, during the exercise protocol are restored within 4 d.

In conclusion it is shown that the set-up described here permits valid repeated measurements of contractile work within the same rats if electrical stimulation via percutaneous needle electrodes is used. Furthermore, it is concluded that potential metabolic abnormalities, due to the high-intensity of the exercise protocol, are restored within 4 days following the first exercise session. Since contractile work has a low variance (if expressed per gram active muscle mass) it is theoretically possible to detect minimal effects of interventions (e.g., dietary manipulation and ergogenic aids) on muscle contractile work.

During the fixed frequency (FF) protocol a rapid drop in muscle contractile work was observed during the first 350 contractions. This rapid decline in contractile work from $20 \mathrm{~J} \cdot \mathrm{kg}^{-1} \mathrm{~mm}$ to approximately $6 \mathrm{~J} \cdot \mathrm{kg}^{-1} \mathrm{~mm}$ during the first 350 contractions was originally hypothesized to reflect a fall in the concentration of directly available high-energy phosphates. (ATP and PC). Following a rapid fall, contractile work plateaued. During the plateau phase muscle glycogen was believed to be the main substrate. Analyses of muscle glycogen content in the exercised muscles, however, showed that the rapid fall in contractile work was accompanied by a rapid decline in muscle glycogen. After 350 contractions, when contractile work had just begun to plateau, muscle glycogen had fallen from 164.6 $\mu \mathrm{mol} \cdot \mathrm{g}^{-1} \mathrm{ww}$ pre-exercise to $50.1 \mu \mathrm{mol} \bullet \mathrm{g}^{-1} \mathrm{ww}$ after 350 contractions while only minor changes in muscle glycogen were observed during the final 2000 contractions (39.1 $\mu \mathrm{molog}^{-1} \mathrm{ww}$ after 2400 contractions).

In an attempt to prevent the rapid breakdown of muscle glycogen during the early phase of exercise, the fixed work (FW) protocol was developed. During this protocol the frequency of stimulation was gradually increased until work equaled $-20 \%$ of maximal work, meeting the level where work plateaued in the FF protocol. Although the rate of glycogen breakdown during the first 350 contractions tends to be less rapid in the FW protocol than in the FF protocol, muscle glycogen content after 2400 contractions was even lower in the FW protocol. Part of the rapid decline in muscle glycogen may originate from the fact that the muscle group studied here (TA and EDL muscle) is predominantly a fast glycolytic muscle $(52 \%$ IIA and $46 \%$ II fibers, Fig 2.7). 


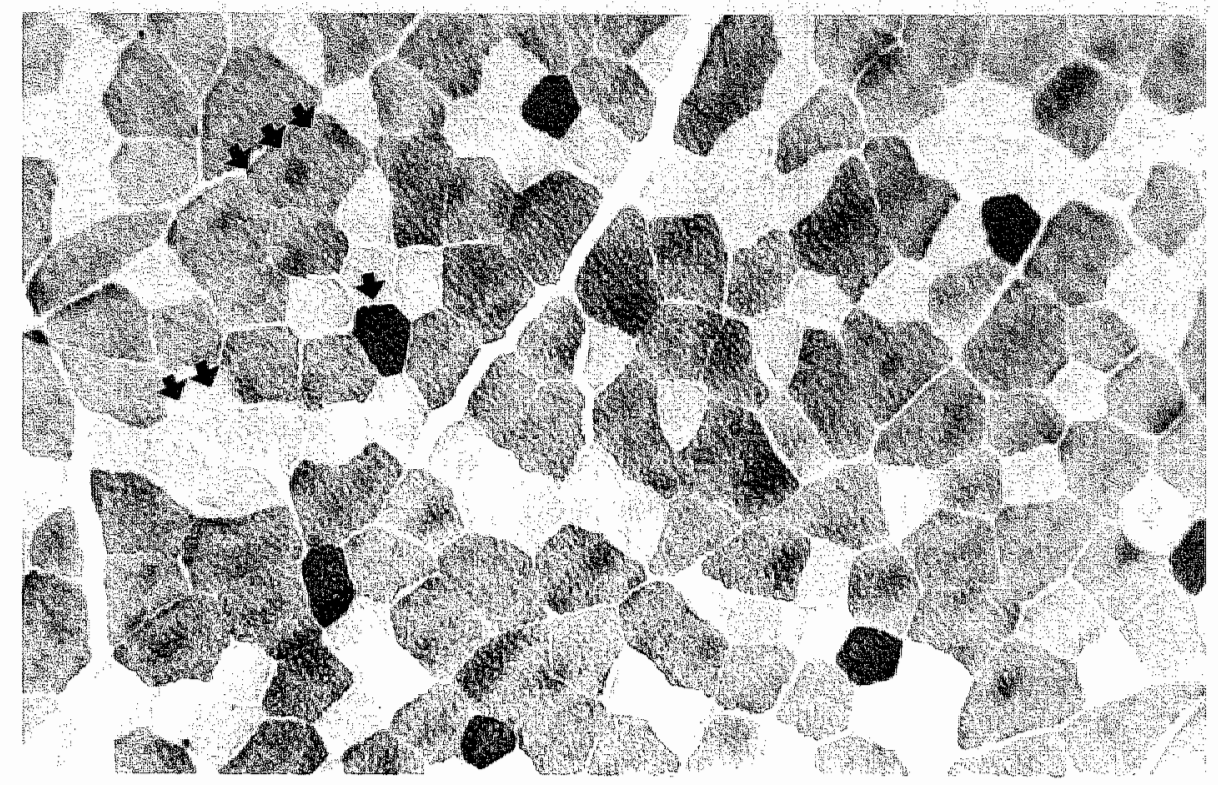

Figure 2.7: Typical ATP-ase (pH 4.5) stain of the midbelly region of rat tibialis anterior muscle showing only a tew $(<2 \%)$ type I fibers $(\uparrow),-58 \%$ type lla fibers $(\uparrow+1)$ and $\sim 40 \%$ type llb fibers $(\uparrow+\uparrow)$.

Despite low muscle glycogen levels, contractile work was maintained during the final 2000 contractions. We hypothesize that, during the final 2000 contractions, a mixture of blood glucose, fatty acids and intramuscular triglyceride stores provides the exercising muscle with energy. Maintenance of blood glucose levels during exercise is regulated by glycogenolysis of liver glycogen. Since the mass of the exercised muscle group is relatively smalli $(\sim 850 \mathrm{mg})$ it is unlikely that depletion of liver glycogen stores takes place during the exercise protocol of 2400 contractions. Therefore, the possibility arises that relatively high work values can be maintained despite low glycogen and that glycogenolysis of liver glycogen partly fuels muscle contraction.

It is concluded that the rapid and profound decline in contractile work and muscle glycogen do not correspond to what is observed during endurance exercise. More research is needed in order to develop valid endurance protocols in the ratdynamometer. It is not unlikely that instead of using the TA-EDL muscle group a switch to a more oxidative muscle group (e.g. \#riceps surae) is necessary for development of valid endurance protocols.

Fiber strain velocity measurement

Ankle angular velocity was related to muscle fiber strain velocity during passive rotation (without muscle contraction) as well as during active rotation (with muscle contraction). The data on muscle fiber strain velocity are linearly related to the ankle angular velocity under the testing conditions. It has to be emphasized that the number of frames recorded is inversely related to ankle angular velocity. Video recordings typically have a sample frequency of $25 \mathrm{~Hz}$, implicating that e.9. 25 
frames are recorded at $50^{\circ} \cdot \mathrm{s}^{-1}$ and only 2 at $500^{\circ} \cdot \mathrm{s}^{-1}$. Separation of odd and even fields doubles the temporal resolution, thus increasing the number of recorded frames to 4 at $500^{\circ} \cdot \mathrm{s}^{-1}$.

Fiber strain velocity under active conditions is $\sim 1.5$ times higher than under passive conditions. This apparently contradictory result is explained by the fact that muscle fibers shorten during the $210 \mathrm{~ms}$ fixed-end 'isometric' pre-contraction. During this tetanic pre-contraction the distance between the spheres placed on the aponeurosis increased while the distance between the spheres placed on the contractile tissue declined (Fig 2.7). During active rotation aponeurosis segment length remained constant while the muscle fiber segment length increased. Under passive conditions both muscle segment and aponeurosis segment were lengthened. Since the range of motion was identical under both active and passive conditions, lengthening of the muscle segment under passive conditions was less compared to muscle segment lengthening under active conditions. As a consequence, muscle fiber strain velocity under active conditions was higher than under passive conditions. All experimental data presented in the present thesis were obtained with an ankle velocity of $500^{\circ} \cdot \mathrm{s}^{-1}$, corresponding to a fiber strain velocity during lengthening contractions of $1.4 \mathrm{~L}_{*} \mathrm{~s}^{-1}$.

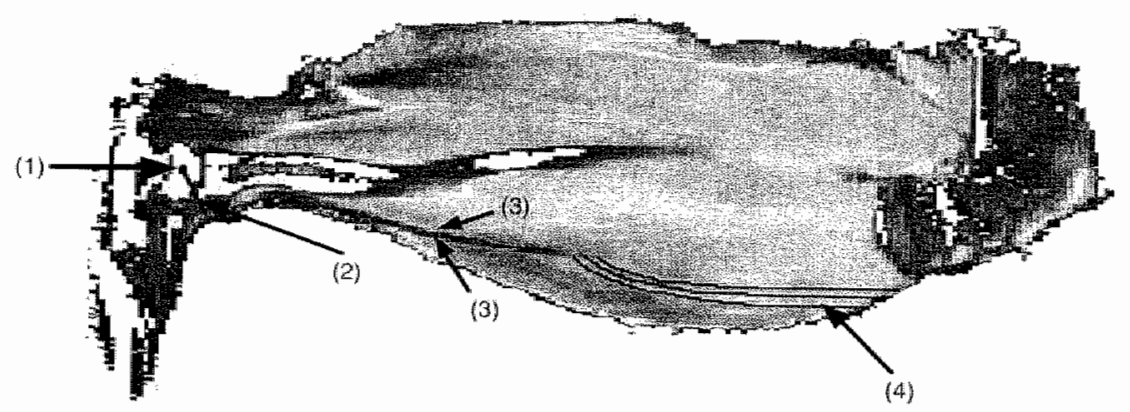

Figure 2.8: High resolution Magnetic Resonance Image of a transversal midsagittal section of a rat lower hindlimb. Center of rotation of the ankle joint (1) and the lever arm (2) of the TA muscle are marked. Arrowheads point at the distal tendan of the tibialis anterior muscle (3). Mean fiber length was based on the 3 fibers indicated in the picture (4). Bar represents $10 \mathrm{~mm}$.

An attempt was made to compare fiber strain velocity calculated using the formula presented here, with fiber strain velocity based on a formula presented by Ashton-Miller et al. (1). In the Ashton-Miller equation, originally formulated to calculate fiber strain velocities in mice, the required input parameters are: muscle lever arm (in millimeter), angular velocity $\left({ }^{\circ} \cdot \mathrm{s}^{-1}\right)$, pennation angle (degrees) and fiber length (in millimeter). Lever arm and fiber length were estimated based on the results of high resolution MRI measurements on rat lower hindlimb (Fig 2.8). Since estimation of pennation angle from the MRI picture would not give reliable results and fiber strain velocity is only affected by the cosine of the angle of pennation, the effect of angle of pennation on fiber strain velocity was neglected. Muscle lever arm 
was estimated to be $2.77 \mathrm{~mm}$ (Fig 2.8) while fiber length was calculated to be 16.3 mm based on the mean length of the 3 fibers marked in the MRI picture.

Considering an ankle velocity of $500^{\circ} \cdot \mathrm{s}^{-1}$, the computed fiber strain velocity using the Ashton-Miller formula (1) equaled $1.44 \mathrm{Lf}^{-1} \mathrm{~s}^{-1}$ "this corresponds well with the fiber strain velocity calculated based on the linear relationship between ankle angular velocity and fiber strain velocity discussed in the present manuscript (1.4 $\left.L_{f} / s\right)$. We conclude that the formula on fiber strain velocity presented by AshtonMiller for mice can also be applied to rats. Generalization of both FSV equations presented here to other muscles or species may be biased by the fact that muscle geometry is not taken into account in both formulae presented here. Besides, the construction of the rotational parts and (mal)alignment of the ankle rotation axis with the rotation axis of the set-up, may affect muscle fiber strain velocity if measured with the video system described here. Consequently, application of the formulae on FSV from the present study to other set-ups should be done with care. However, the method presented here to measure FSV is valid and can be easily performed in other experimental set-ups.

In conclusion, the rat dynamometer discussed here, provides accurate and valid in situ measurement of external work of rat hindlimb dorsiflexor muscles during lengthening and shortening contractions at ankle velocities ranging from 50$500^{\circ} \cdot \mathrm{s}^{-1}$. Inter-subject variability is low, intra-subject variability is minimal if stimulation is performed using well-positioned and tightly-fixed percutaneous needle electrodes. Because of the non-invasive character of the present set-up, combined with needle electrode stimulation, valid and accurate repeated measurements can easily be performed. Muscle fiber strain velocity was found to be linearly related to ankle angular velocity under passive as well as under active conditions and matched well with computed muscle fiber strain velocity based on a formula provided by others combined with high resolution MAI derived muscle parameters.

\section{Acknowledgment}

The authors are very grateful to the engineers $E$. Dekkers and $R$. Janssen from the Eindhoven University of Technology for their skillul technical assistance whille designing, building and testing the ratdynamometer presented here. R.P. Hesselink is gratefully acknowledged for excellent fiber typing of rat TA muscle. The authors thank K.J. Hommels for the illustration depicted in Figure 2.1. 


\section{References}

1. Ashiton-Miller, J.A, Y. He, U. A. Kachiresan, D. A. McCubbrey, and J. A. Faullkner. An apparatus to measure in vivo biomechanical behavior of dorsi- and plantartexors of mouse ankile. J Appt Physiol 72: 12051211,1992

2. Faulkner, J. A., D. A dones, and J.M. Found. Injury to skeletal muscles of mice by forced lengthening during contractions. Q.J Exp Physiol 74: 661-670, 1989.

3. Geusen, M. De relatie tussen hoeksnelheid van het enkelgewt tht en de vezelreksnellheid van de Tibialis Anterior wan een rat. Eindhowen University of Techinalogy, 1996.

4. Hawkins, D., and M. Bey. A comprehensive: appraach for studying muscle tendon mechanics. $J$ Biomech Eng 116: 51-55, 1994.

5. Lännergen $\mathrm{J}_{\mathrm{s}}$, and $\mathrm{H}$. Westerblad. Force decline due to tatigue and intracellular acidification in isolated fibres from mouse skeletal muscle. J Physiol 434: 307322, 1991.

6. Lieber, R. L., and J. Friden. Muscle damage is not a function of muscle force but active muscle strain. 4 Appl Physid 74: 520-526. 1993.

7. Lieber, R. L., M. C. Schmitz, D. K. Mishra, and J. Fridén. Contractile and cellutar remodeling in rabbil skeletal muscle after cyclic eccentric eccentric contractions. J Appl Physiol 77": 1926, 1994.

8. McCully, K. Ka, and J. A. Faulkner, nnjury to skeletal muscle fibers of mice following lengthening contractions. J' Appl Physiol 59: 119-126, 1985.

9. wan Baval, H., M. R. Drost, J. D. Wielders, J. M. Huyghe, A. Husan, and J. D. Janssen. Strain distribution on rat medial gastrocnemius (MG) during passiwe strelch. J Biomech 29: 1069.74, 1996.

10. Warren, G. L., D. Hayes, D. A. Lowe, J. H. Williams, and A. B. Armstrong. Eccentric contraction.induced injury in normal and hindlimb-suspended mouse soleus and EDL muscles. I Appl Physiol 77: 1421-1430, 1994 . 


\section{3}

\section{Structural Muscle Damage and Muscle Strength}

After Incremental Number of Isometric and Forced Lengthening Contractions

M.K.C. Hesselink, H. Kuipers, P. Geurten, H. van Straaten 1

Journal of Muscle Research and Cell Motility, 17, 335-341, 1996.

1 Department of Anatomy/Embryology, Maastricht University. 


\begin{abstract}
Exercise-induced muscle damage is characterized by histological changes, like Zline streaming, inflammatory response and decreased muscle function reflected in a prolonged decline in maximal isometric muscle strength after lengthening contractions. It is assumed that force decrement is mainly related to the amount of structural damage. However, the relationship between number of lengthening contractions, magnitude of structural damage and force decrement is not very well documented. Therefore we studied the effect of an increasing number of both isometric and forced lengthening contractions on histological parameters of muscle damage and maximal isometric force in an experimental in situ rat model. Tibialis anterior (TA) muscles of male Wistar rats were subjected to an increasing number of either isometric or lengthening contractions and were examined for histological markers of muscle damage. The present study shows that muscle damage increases progressively with the number of forced lengthening contractions. Maximal isometric torque was found to decline after both types of exercise. However, the decline after forced lengthening exercise was more pronounced. Only a weak relationship between percentage of histological muscle damage and isometric torque after forced lengthening contractions was found. The findings of the present study suggest that the decline in muscle force after lengthening exercise may partly be attributed to other factors than structural damage.
\end{abstract}




\section{Introduction}

Unaccustomed or strenuous exercise may lead to muscle damage and delayed onset muscle soreness (DOMS). Exercise induced muscle damage and associated soreness is commonly reported after eccentric or lengthening muscle contractions such as downhill running (3). In experimental animal models muscle damage can be induced by downhill running in conscious animals and by forced lengthening contractions in anaesthetized animals $(3,10,11,16)$. Assessment of injury by histological features, such as Z-line streaming and inflammatory response, has previously shown that the extent of tissue injury after forced lengthening contractions initially increases in proportion to the number of contractions. However, McCully and Faulkner reported no further increase in number of affected muscle fibers when the forced lengthening exercise is continued for a longer period of time (10) while van der Meulen et al. reported a disproportional increase in muscle damage in rats that ran $2.5 \mathrm{~km}$ compared to rats running $1.5 \mathrm{~km}(17)$. These different results are most probably attributable to fatigue originated by differences in the exercise protocols, short- term high intensity exercise in McCully and Faulkner's study (10) versus prolonged moderate intensity exercise in the study by van der Meulen (17). Muscle damage is reflected in a decrement of maximal isometric strength which is known to remain depressed for several days after exercise (11). Also in humans lengthening exercise-induced muscle damage is associated with a decline in maximal voluntary contraction force (MVC) $(4,5,13)$. It is assumed that the force decrement is mainly related to the amount of structural damage. However, little data exist about the relationship between number of lengthening contractions, the amount of structural damage and related force decrement. Therefore the aim of the present study was to investigate the maximal isometric force and amount of histological damage after an increasing number of forced lengthening contractions and isometric contractions. To diminish fatigue, the forced lengthening and the isometric contractions were given in 3 minute bouts interspaced by 5 minutes recovery.

\section{Materials and methods}

Male Wistar rats (Charles River), aged 12-14 weeks with an average body weight of $312 \pm 24.2$ grams (mean \pm S.E.M.) were used in this study. Surgical procedures, exercise and muscle dlissection were performed under pentobarbital anesthesia (Narcovet ${ }^{(B)} 0.1 \mathrm{ml} / \mathrm{kg}$ body weight, i.p. injection) after short-term (<20 seconds) $\mathrm{CO}_{2}$ sedation.

Surgical procedures

To expose the common peroneal nerve a small incision just distal to the patella at the lateral side of the hind limb was made. Subcutaneous tissue was carefully separated and the uncoated tip of a stainless steel electrode was hooked to the nerve while the other tip was fixed to the skin. To prevent dehydration during exercise the incision was closed with one single suture. After exercise the electrode was carefully removed and the underlying tissue and skin were sutured. Twenty-four hours after terminating the exercise the tibialis anterior (TA) muscle of both legs was dissected and processed for histological examination. Immediately afterwards the rats were sacrificed by cervical dislocation. 


\section{Exercise protocol}

During the exercise period the rats were placed on their back in the experimental set-up. The tibia was fixed at its most proximal part, at the lateral and medial condyle. The foot was fixed in a special designed shoe connected to a foot plate, to keep the foot either in a predetermined position (isometric exercise) or, in case of forced lengthening exercise, to allow rotational movement in the ankle joint (plantar and dorsiflexion). Tetanic contractions were induced by electrical stimulation via the electrode using 300 milliseconds pulse trains with a frequency of $150 \mathrm{~Hz}$ and a charge of $3 \mathrm{~V}$. During isometric exercise and isometric torque measurements, the angle between foot and tibia was maintained at $90^{\circ}$. During forced lengthening (eccentric) contractions the initial angle of the ankle joint was $80^{\circ}$. Using a pneumatic system the dorsiflexors were forcibly lengthened with a fiber velocity of about 1.1 fiber lengths per second until the angle of ankle joint was $130^{\circ}$. This rotational movement of the foot was initiated $200 \mathrm{~ms}$ after starting the stimulation of the nerve to ensure an adequate muscle tension before lengthening. Before the passive return stroke (back to $80^{\circ}$ ) was initiated, muscle contraction sustained isometrically at $130^{\circ}$ for $50 \mathrm{~ms}$. In all rats both hind limbs were successively exercised in randomized order, one side isometrically and the other side eccentrically. The muscles were stimulated in bouts of 3 minutes. During each exercise bout every 3 seconds a tetanic contraction of $300 \mathrm{~ms}$ was employed. This resulted in 60 contractions per exercise bout. During the time span between subsequent isometric contractions no exercise was performed while after forced lengthening only passive exercise was performed during the return stroke back to $80^{\circ}$ (return stroke was performed with a fiber velocity of about 0.5 fiber lengths per second). Immediately before and after every exercise bout isometric torque of the dorsiflexor muscles was measured using a sensitive, precalibrated force transducer (Grass FTO3C). The rats were subjected to 1 to 5 bouts, consisting of either $60,120,180,240$ or 300 isometric or lengthening contractions, respectively. Subsequent exercise bouts were interspaced by a 5 minute recovery period. In each group 12 TA muscles were examined ( 6 isometrically exercised muscles and 6 muscles subjected to lengthening exercise. The sham operated control group (4 rats, 8 TA muscles) underwent all surgical procedures and were placed in the experimental set-up without doing any exercise.

Tissue sampling and processing

Twenty-four hours, after exercise the TA muscles were removed, the mid belly region was isolated and rapidly frozen in dry ice cooled isopentane. Collected muscle samples were stored at $-80^{\circ} \mathrm{C}$ until processing. Transverse cryosections of $10 \mu \mathrm{m}$ were cut at $-20^{\circ} \mathrm{C}$ (Anglia Scientific), stained with hematoxylin-eosin (HE) to study histological features of injury and examined microscopically (Zeiss) for inflammatory changes. Muscle fibers were considered to be damaged when they were infiltrated with mononuclear cells (3), had multiple central nuclei (6) or had a swollen appearance (12). Three serial transverse sections per TA muscle were examined, the mean of these 3 sections was considered to give representative information about the amount of damage in the mid-belly region of the examined muscle (17). Muscle damage was quantified by point counting using a $10^{*}$ ocular mounted grid with $10 \cdot 10$ squares and a Zeiss Neofluar $10^{*}$ objective (0.32 N.A.) 
(one square equals $100 \cdot 100 \mu \mathrm{m}$ ). Per section, at least 30 grids were counted. Muscle damage was expressed as percentage of grid squares containing affected muscle fibers.

\section{Statistics}

Data on isometric torque before and after each exercise bout were compared using repeated measures ANOVA with Scheffer post-hoc tests, while the data on muscle damage were analyzed with factorial ANOVA with Scheffé post-hoc tests. Unpaired students T-tests were performed to compare between the isometric and the lengthening exercised group. The level of significance was set at $p<0.05$ unless stated otherwise. All results are presented as mean \pm S.E.M..

\section{Resullts}

\section{Morphological features}

None of the sham treated muscles showed histological indices of muscle damage (infiltration of mononuclear cells, central nuclei or a swollen appearance) or deviations from the normal pattern seen in transverse sections of the TA muscle. Isometric exercise showed no significant changes in muscle morphology, although occasionally some muscle fibers that were subjected to 300 isometric contractions showed a slightly swollen appearance (Fig 3.1d). However, lengthening exercise resulted in progressive morphological damage with increasing number of contractions, the level of significance being reached at 180 forced lengthening contractions (see Table 3.1 and Fig $3.1 \mathrm{~b}$ and $3.1 \mathrm{c}$ ).
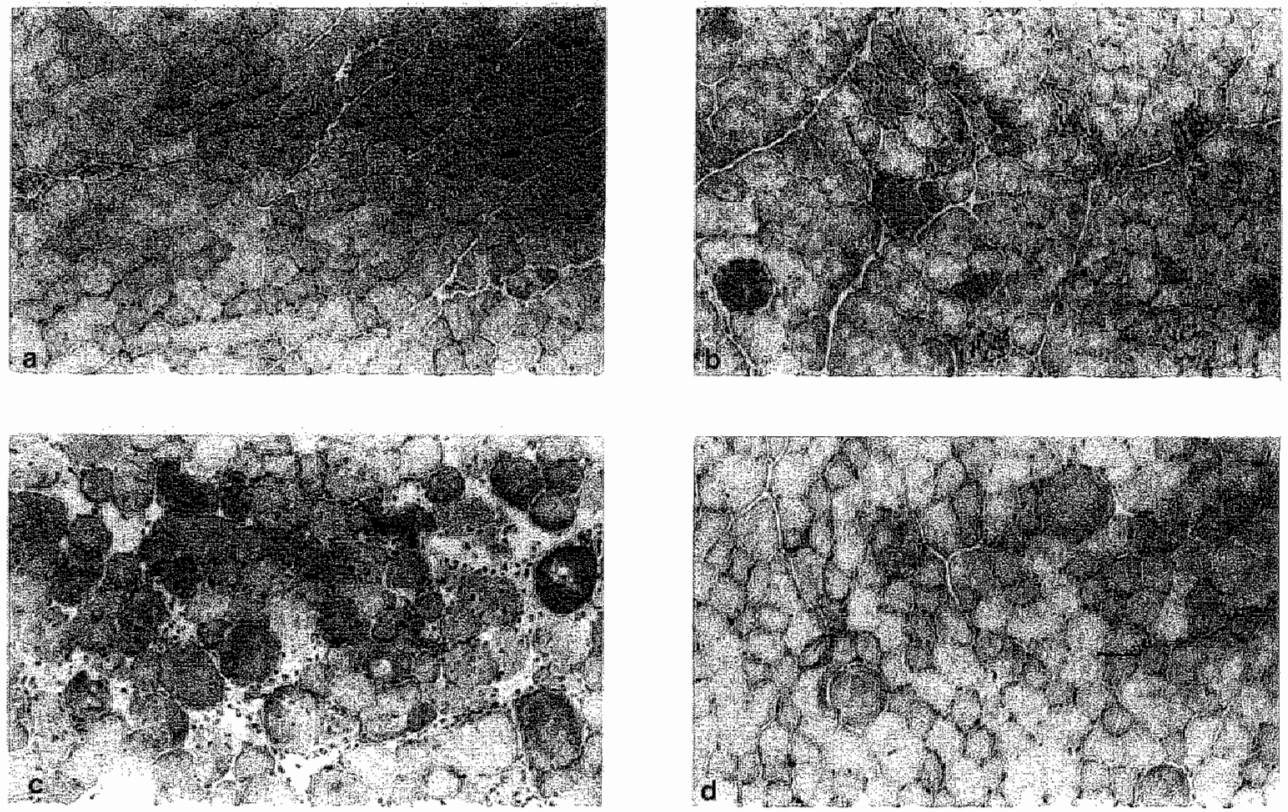

Figure 3.1: representative photomicrographs of cross sections of TA muscle 24 hours after 60 (a), 180 (b), or 300 forced (c) lengthening contractions or 24 hours after 300 isometric contractions (d). 
Furthermore, it was abvious that 300 lengthening contractions induced significantly more damage than any of the preceding exercise bouts while 180 or 240 lengthening contractions only induced more damage than was abserved after 0 or 60 lengthening contractions (Fig 3.1a and Fig 3.2). Regression analysis of percentage of damage against number of contractions showed an $R^{2}$ of 0.79 $(p<0.0001)$ (Fig 3.2).

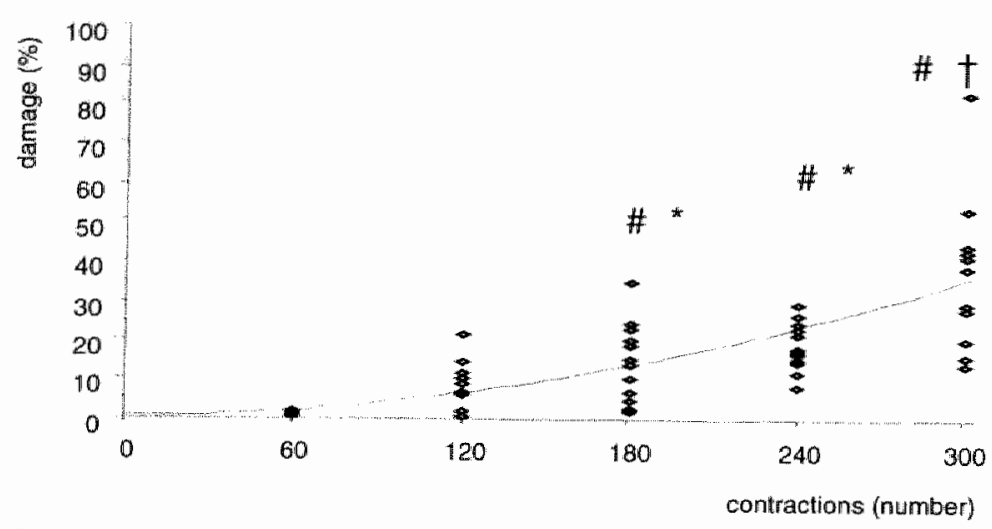

Figure 3.2: Regression plot of percentage of grid blocks containing damaged muscle fibers against number of farced lengthening contractions $\left(R^{2}=0.79\right)$. \# is different from 0 contractions, ${ }^{*}$ is different from 0 and 60 contractions, $\dagger$ is different from all preceding exercise bouts $(p \leq 0.05)$.

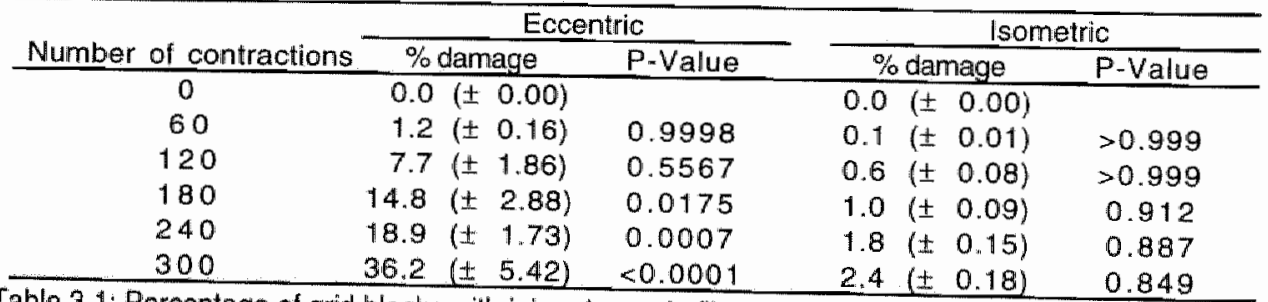

Table 3.1: Percentage of grid blocks with injured muscle fibers (mean \pm SEM) related to contraction type and number with corresponding $p$-values when compared to zero contractions.

\section{Force measurements}

No difference in pre-exercise isometric torque between the isometric and lengthening exercised group was found. Therefore the initial value of isometric torque was set at $100 \%$. Due to technical problems we were unable to measure isometric torque after exercise in the group that was subjected 300 contractions. With increasing number of contractions a progressive decline in isometric torque both in the lengthening exercised group and in the isometric exercised group occurred. After each exercise bout isometric torque after lengthening exercise was lower than after isometric exercise. This difference in isometric torque was significantly different after 60 and 120 contractions. When the number of contractions was increased, the decline in isometric torque was leveling off in both groups (Fig 3.3). Regression analyses of isometric torque against number of 
contractions showed an $R^{2}$ of 0.80 for the isometric exercised leg and 0.89 after lengthening exercise, while the low P-value $(<0.001$ for both isometric and lengthening exercise) indicates a high fit of the regression line (Fig 3.3). Isometric torque 5 minutes after termination of each exercise bout was not significantly different in the isometric exercised group ( $P=0.1302)$.

In the lengthening exercised group, isometric torque 5 minutes after termination of each exercise bout gradually decreased significantly from $50.2 \%$ after 60 contractions to $28.2 \%$ after 240 contractions (Table 3.2). A weak ( $R=$ -0.593 ), but significant, negative correlation was found between the percentage of damage and isometric torque in the lengthening exercised group. Since isometric exercise did not lead to muscle damage as defined before, no such correlation was found between isometric exercise and isometric torque.

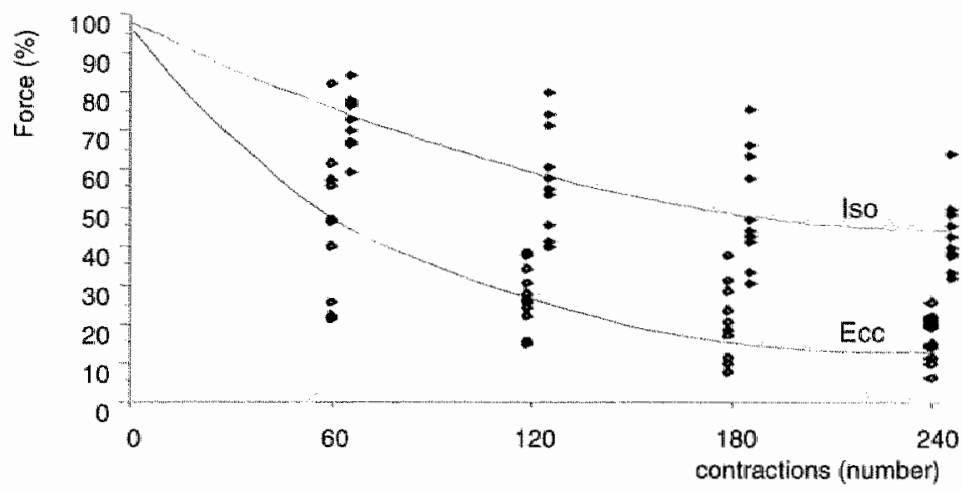

Figure 3.3: Regression plot of isometric torque after increasing number of either isometric (Iso) contractions or forced lengthening contractions (Ecc).

\begin{tabular}{|c|c|c|c|c|}
\hline \multirow{2}{*}{$\begin{array}{l}\text { Number } \\
\text { of contractions }\end{array}$} & \multicolumn{2}{|c|}{ Eccentric } & \multicolumn{2}{|c|}{ Isomelric } \\
\hline & Prewexercise bout & Post-exercise bout & Pre-exercise bout & Posl-exercise bout \\
\hline 60 & $100( \pm 0.00)$ & $47.9( \pm 5.24)$ & $100( \pm 0.00)$ & $74.2( \pm 2.02)$ \\
\hline 120 & $50.2( \pm 5.41)$ & $27.1( \pm 2.44)$ & $78.2( \pm 3.31)$ & $59.9( \pm 3.66)$ \\
\hline 180 & $35.1( \pm 3.17)$ & $22.3( \pm 2.74)$ & $71.4( \pm 4.35)$ & $52.0( \pm 3.91)$ \\
\hline 240 & $28.2( \pm 3.29)$ & $17.8( \pm 1,70)$ & $65,3( \pm 4,20)$ & $43.4( \pm 3.09)$ \\
\hline
\end{tabular}

Table 3.2: Isometric torque (percentage of intiall torque, mean \pm SEM).

\section{Discussion}

The objective of the present study was to assess the relationship between number of forced lengthening contractions, amount of structural damage 24 hours after exercise and isometric torque. In this study we examined the inflammatory response as indicator of structural damage 24 hours post-exercise since at this time point the amount of histological damage has reached its peak (15). Muscle fibers were considered to be affected when they were infiltrated with mononuclear cells (3), had multiple central nuclei (6) or were swollen (12), see Figure 3.1d. Consistent with findings of others using comparable exercise protocols $(10,11,16)$, we observed inflammatory and degenerative changes in the muscles that were subjected to 120 or more forced lengthening contractions. However, no such 
changes were found either in the sham-operated controls or in the isometrically exercised muscles. As can be seen in Figure 3.2, less than 120 contractions did not induce a significant increase in injured muscie fibers. Regression analysis showed that the amount of muscle damage increased with the number of contractions. This is not consistent with findings of McCully and Faulkner (10) who subjected the EDL muscle of mice to an incremental forced lengthening protocal at 3 different fiber velocities $\left(0.2,0.5\right.$ and $\left.1.0 \mathrm{~L} \cdot \mathrm{s}^{-1}\right)$. After 5 minutes of exercise (corresponding with $\$ 50$ forced lengthening contractions) with a velocity for lengthening contractions of $1.0 \mathrm{Lf} \mathrm{L}^{-\mathrm{s}^{-1}}$, McCully and Faulkner found a leveling off of the amount of damage. Even when the number of contractions was increased up to 450 , the increase in injured muscle fibers remained approximately $30 \%$, while in our study the amount of injured muscle fibers was still increasing when the exercise was prolonged. According to the regression analysis, the calculated curve fits well $\left(R^{2}=0.79\right.$, $P<0.0001)$ and indicates a further increase in damage with an increasing number of contractions. The reason for this discrepancy between McCully's study (10) and ours may be attributed to differences in fatigue. In contrast to the present study McCully and Faulkner (10) did not implement recovery periods after a number of contractions, therefore fatigue might have led to a lower tension in the forcibly lengthened muscles thus preventing structural damage. Since McCully studied the EDL and we studied the TA it can also be argued that the delayed rise in damage in our study can be attributed to the difference in length of muscle fibers and associated strain. Lieber and Fridén (8) showed in TA muscles of the New Zealand White rabbit that muscle damage is related to active muscle strain rather than to muscle force. This means that for a given joint rotation short muscle fibers need to lengthen more than longer muscle fibers. If this is applied to the TA and EDL muscle in rabbits it seems that the TA fibers are about twice as long as the EDL fibers (7), since both muscles have about the same lever arm at the ankle joint, this means that for a given joint rotation the active strain of EDL fibers is more than in the myofibers of the TA and are therefore possibly more susceptible to damage than the TA fibers. Since Lieber studied rabbits (8), McCully studied mice (10) and we studied rats, the comparison of the results is biased by species differences and is therefore speculative. Further studies of fiber length and lever arm of rat TA and EDL muscle are required to find out whether the differences in susceptibility to muscle damage between EDL and TA myofibers are in fact related to differences in muscle strain.

Lengthening exercise has previously been shown to induce decreased muscle strength in animal models $(8,11)$, as well as in humans $(4,5,13)$. In the present study isometric torque immediately after the first bout of 60 forced lengthening contractions declined to $47.9 \%$ of its initial value whille after isometric exercise a decline to only $74.2 \%$ was found. This decline in torque is quantitatively comparable to findings in humans after lengthening exercise (5). The deficit in isometric torque between values recorded after lengthening and isometric exercise is approximately $30 \%$ after successive exercise bouts. This deficit in torque is present already after the first bout of 60 contractions. At this time, however, only minor histological changes are reported after 60 forced lengthening contractions (Table 3.1). Therefore, we postulate that muscle damage plays a minor role in the 
deficit in muscle torque observed between isometrically and forcibly lengthened muscles. This postulation is supported by the weak $(R=-0.593)$ correlation between the extent of muscle damage and post-exercise recorded isometric torque. The discrepancy between magnitude of histological muscle damage and force decrement has been observed by other investigators as well $(9,18)$. This indicates that other factors than myofibrillar damage must be taken into account when looking at muscular performance after lengthening contractions. Some investigators attribute the force decrement partly to a failure of the excitationcontraction coupling (19). One of the contributing factors for decrease in force may also be a metabolic deterioration. Although work performed during lengthening is supposed to be less energy consuming than concentric or isometric exercise (14) it can be challenged whether this also counts for forced lengthening contractions. The lower energy cost during lengthening exercise is commonly attributed to a fewer number of muscle fibers that are active during lengthening contractions (1). Since during tetanic stimulation all motor units are recruited there is little reason to assume lower energy cost during tetanic forced lengthening contractions compared to tetanic isometric contractions. One of the initial events during forced lengthening exercise is disruption of the sarcolemma permitting calcium influx into the exercised muscles in excessive amounts successively impairing normal metabolism and concomitant muscular performance (2). Increased IMP levels after lengthening exercise, together with a decreased ATP content (upto $24 \mathrm{~h}$ postexercises), have been reported by van der Meulen et al. (16) using a similar model. These observations point towards deranged energy metabolism. However, more research is needed to study whether changes in the metabolic state of forcibly lengthened muscles are more closely related to decreased muscle strength than are histological indices of damage.

In conclusion, the amount of lhistological muscle damage increases progressively with the number of forced lengthening contractions. However, force development showed a different pattern. A decline in isometric torque both after isometric exercise and after forced lengthening exercise was found, the decline in the latter however was more pronounced and isometric torque after every exercise bout was lower after forced lengthening exercise than it was after isometric exercise. Only a weak relationship between percentage of muscle damage and isometric torque after forced lengthening contractions was found. This suggests that structural damage plays no major role in decline in muscle performance after lengthening exercise.

\section{Acknowledgment}

The skillful technical assistance of Paul van Dijk during cutting and staining procedures is gratefully acknowledged. 


\section{References}

1. Abbott, B. C., B. Bigland, and d. M. Ritchie. The physiological cosit of negative work. I Physiol 117: 380 390,1962

2. Armstrong. R. B. Initial events in exercise-induced muscular injury. Med Sci Sports Exerc 22: 429-435, 1990.

3. Amstrong, R. B., R. W. Oglivie, and J. A. Schwane. Eccentric exercise induced injury to skeietal muscle. $J$ Appl Physitiol 54: 30-93, 1983.

4. Clarkson, K. Nosaka, and B. Braun. Muscle function after exercise-fnduced muscle dlamage and rapid adaptation. Med Sci Sponts Exerc 24: 512-520, 1992.

5. Cleak, M. J., and R. G. Eston. Muscle soreness, swelling, stiffness and sirength loss after eccentric exercise. Br u Sp Med 26: 267-272, 1992.

6. Kuipers, H., J. Drukker, P. M. Frederik, P. Geurten, and $G$. van Kranenburg. Muscle degeneration after exercise in rats. Int J Sports Med 4: 49-55, 1983.

7. Lieber, R. L., and F. T. Blevins. Skeletal muscle architecture of the rabbit hindlimb: functional implications of muscle design. J Morphol 199: 93-101, 1989.

8. Lieber, R. L., and J. Friden. Muscle damage is noll a function of muscle force but active muscle slrain. $\checkmark$ Appl Physiol 74: 520-6, 1993

9. Lieber, R. L., T. M. Woadburn, and J. Friden. Muscle damage induced by eccentric contractions of $25 \%$ strain. J Appol Physiol 70: 2498-507, 1991.

10. McCully, $K, K_{n}$ and J. A. Faulkner. Characteristics of lengthening contractions associated with injury to skeletelal muscle tibers. J Appol Physiol 61: 293-299, 1986.

11. McCully, K. K., and J. A. Faulkner, Injury to skeletal muscle fibers of mice following lengthening contractions. J Appl Physiol 59: 119-126, 1985.
12. Peeze-Binkhorst, F. M. D. W. Slaat, H. Kuipers, G.-J. Tangelder, and R. S. Reneman. Exercise-induced swelling of rat soleus muscle: its relationship with intramuscular pressure. J App/ Physiol 69: 67-73, 1990.

13. Rodenburg, J. B., P. R. Bar, and B. R. De. Relations between muscle soreness and biochemical and functional outcomes of eccentric exercise. I Appl Physid 74: 2976-83, 1993.

14. Stauber, W. T. Eccentric action of muscles: Physiology, iniury, and adaptatian. Exerc Sports Sci Rev 17: 157-185, 1989.

15. van der Meulen, J. H. Exercise-induced muscle damage: morphological, biochemical and functional aspects. Rijksuniversiteit Limburg, Maastricht, 1991.

16. van der Meulen, J. H. H. Kuipers, F. R. M. Stassen, H. A. Keizer, and G. J. van der Vusse. High energy phosphates and related compounds, glycogen levels and histology in the rat tibialis anterior muscle atter forced lengthening and isometric exercise. Pflügers Arch 420: 354-358, 1992.

17. van der Meulen, J. H. , H. Kuipers, J. C. wan derWal, and J. Drukker. Quantitative and spatial aspects of degenerative changes in rat soleus muscle after exercise of different durations. J Anat 182: 349-353, 1993.

18. Warren, G. L., D. Hayes, D. A. Lowe, J. H. Williams, and $\mathrm{F}$. B. Armstrong. Eccentric contraction-induced injury in normal and hindlimb-suspended mouse soleus and EDL muscles. J/App/ Physiol 77: 142\%-1430, 1994.

19. Warren, G. L., D. A. Lowe, D. A. Hayes, C. J. Karwaski, B. M. Prior, and R. B. Armstrong. Exciation lailure in eccentric contraction-induced injury af mouse soleus muscle. J Physiol Landon 468: $487-499_{11} 1993$. 
Acute and Sustained Effects of Isometric and Lengthening Muscle Contractions on High-Energy Phosphates and Glycogen Metabolism in Rat Tibialis Anterior Muscle

M.K.C. Hesselink, H. Kuipers, H. A. Keizer, M. R. Drost, G. J. van der Vusse 1 Journal of Muscle Research and Cell Motility, accepted.

1Department of Physiology, Maastricht University. 


\section{Abstract}

Previous studies have shown that lengthening contractions, in contrast to isometric contractions, readily result in sustained malfunctioning of the exercised muscles. The present study was performed to investigate whether an exercise period with many (240) lengthening contractions results in alterations in muscle high-energy phosphates and IMP content, different from muscles performing a few (60) lengthening or a few (60) or many (240) isometric contractions. Moreover, we sought for possible cause(s) of the inability to replenish muscle glycogen stores following LC. Rat tibialis anterior muscles were subjected in vivo to either 60 or 240 LC or IC. Structural muscle damage occurred only after 240 LC. The fact that tissue glycogen levels declined to a similar extent during LC and IC suggests that the energy demand was comparable during both types of exercise. Nevertheless, the observation that on the one hand tissue stores of adenine nucleotides showed a greater decline and on the other hand that the tissue content of IMP increased to a significantly higher level after LC than after IC, clearly indicates that muscle energy metabolism is more disturbed during $L C$ than during IC. The high tissue levels of IMP may contribute to impaired mechanical function as previously observed in muscles subjected to LC. Tissue glycogen stores and high-energy phosphate levels were not restored to control values $24 \mathrm{~h}$ after $240 \mathrm{LC}$, in contrast to observations $24 \mathrm{~h}$ after $240 \mathrm{IC}$. The present findings indicate that depressed glycogen synthase activity and impaired activity of the mitochondrial marker enzyme cytochrome $\mathrm{C}$ oxidase most likely contribute to a continuous disturbance of energy metabolism in the exercised muscles during $24 \mathrm{~h}$ following $240 \mathrm{LC}$. 


\section{Introduction}

Skeletall muscle contraction can be divided into isometric, concentric or eccentric actions. Under normal physiological conditions lengthening (eccentric) contractions are metabolically less demanding than shortening or isometric contractions when developing the same force (1). Nevertheless, eccentric contractions are associated with a higher susceptibillity for structural muscle damage than concentric or isometric contractions $(2,16,19,20)$. The prolonged decline in muscular contractile performance after an eccentric exercise bout is most likely causally related to muscle damage $(4,5,15,17,25,26,32)$, induced by lengthening of the contracting muscle.

In rat dorsiflexor muscles we previously observed that force generation was compromised bath after successive lengthening (LC) and successive isometric contractions (IC) (12). Force generation was consistently more declined after LC than after IC (12). Part of the decrease in force development after LC can be explained by structural damage inflicted upon the exercised muscles $(12,19)$. However, the decline in force generation in muscles subjected to an exercise bout consisting of $1 \mathrm{C}$ or a limited number of LC (e.g., 60 contractions) is most likelly caused by other factors than structural damage, because the latter two types of exercise did not lead to detectable structural abnormalities in the exercised muscles (12). Suggestions have been made that metabolic factors are responsible for the observed decrease in mechanical performance of the exercised muscle cells $(6,9,18,25,28)$.

The present study was conducted to explore in more detail the metabolic alterations in muscles subjected to an incremental number of lengthening and isometric contractions "both under damaging and non-damaging conditions. Special attention has been paid to the tissue content of glycogen and the tissue levels of high-energy phosphates such as phosphocreatine and ATP. Muscle IMP content was measured as well, as this substance is thought to specifically interfere with proper mechanilcal functioning of skeletal muscle (10). Following this approach, attempts are made to explain the earlier observed decline in force generation in relation to metabolic changes at the end of the respective exercise bouts.

Furthermore, lengthening exercise, leading to structural damage of skeletal muscle cells, has been reported to hamper the post-exercise replenishment of muscle glycogen stores $(6,23,34)$. The reasion for this defect is incompletely understood. Both metabolic abnormalities and enhanced glucose utilization by invading leukocytes have been mentioned as possible factors involved (6). In the present study we investigated whether impairment of glycogen synthase activity might be responsible for the lack of restoration of the endogenous glucose pool during a $24 \mathrm{~h}$ recovery period following extensive lengthening exercise.

In the present study, experiments were performed on rat tibialis anterior (TA) muscles using an identical exercise protocol as used previously when TA muscies were subjected to damaging and non-damaging exercise (12). 


\section{Materials and methods}

Animals

The present study was conducted on 72 TA muscles of 36 male Wistar rats (Charles River), aged 12-14 weeks with an average body weight of $323 \mathrm{~g} \pm 18$ (mean \pm SD). Surgical procedures, exercise and muscle dissection were performed under pentobarbital anesthesia (Narcovet ${ }^{(1)} 1.0 \mathrm{ml} \cdot \mathrm{kg}^{-1}$ body weight, i.p. injection) after short-term, i.e., less than $20 \mathrm{~s}, \mathrm{CO}_{2}$ sedation.

\section{Surgical procedures}

For enabling electrical stimulation, a small incision just distal of the patella at the lateral side of the hindlimb was made in order to expose the common peroneal nerve. Subcutaneous tissue was carefully dissected and the uncoated tip of a stainless steel electrode was hooked to the nerve while the other tip was fixed to the skin, distal to the one hooked to the nerve. During exercise the incision was closed with one single suture. Post-exercise, the electrode was carefully removed and, depending on the protocol, the underlying tissue and skin were either sutured or the TA muscle was dissected immediately post-exercise. After tissue sampling the rats were sacrificed by cervical dislocation.

\section{Exercise protocol}

The experimental procedures and exercise protocol have previously been described in detail elsewhere (12). Briefly, during the exercise period the rats were placed in supine position in the experimental set-up. The experimentall set-up consists of a plateau, a tibia fixation unit and a footplate that can be rotated by a pneumatic system. The tibia was fixed at the most proximal part of the lateral and medial condyle. The foot was fixed in a shoe connected to the footplate. During isometric exercise the shoe and foot were kept in a pre-determined position. Lengthening of the active muscles was induced by rotation of the shoe and foot. Special care was taken to align the axis of plantar-and dorsiflexion with the axis of rotation.

Contractions were induced by electrical stimulation via an electrode using $300 \mathrm{~ms}$ pulse trains (square waves of $0.5 \mathrm{~ms}$ ) with a frequency of $150 \mathrm{~Hz}$ and a voltage of $3 \mathrm{~V}$. During isometric exercise the angle between foot and tibia was maintained at $90^{\circ}$. During lengthening contractions the initial angle of the ankle joint was $80^{\circ}$. Using a pneumatic system the dorsiflexor muscles were forcibly lengthened with an angular velocity of about $1000^{\circ} \cdot \mathrm{s}^{-1}$ until the angle of ankle joint was $130^{\circ}$. To ensure adequate muscle tension at the onset of lengthening, plantarflexion was initiated $200 \mathrm{~ms}$ after starting the stimulation of the nerve. After lengthening of the muscles, a passive return stroke was performed. Dorsiflexor muscles of the left hindlimb were exercised in all rats, while contra-lateral muscles served as non-exercised controls.

Based on the results of a previous study (12), the rats were subjected to either 1 or 4 bouts of 60 IC or LC resulting in 60 or 240 contractions, respectively. All exercised muscles were stimulated in bouts of $3 \mathrm{~min}$. During each exercise bout every $3 \mathrm{~s}$ a tetanic contraction of $300 \mathrm{~ms}$ was employed, resulting in 60 contractions per exercise bout. Subsequent exercise bouts, if any, were interspaced by $5 \mathrm{~min}$ rest. In total, 6 groups of 6 animals each were studied. Muscle metabolic status after exercise was studied by sampling muscle immediately after 
60 and 240 LC and IC. Sustained effects ( $24 \mathrm{~h}$ post-exercise) of the two types of exercise (LC and IC) on muscle metabolic status were studied after 240 contractions only.

During the entire exercise protocol and preceding surgical procedures rectal temperature was monitored and maintained at $37^{\circ} \mathrm{C}$ using a heating pad.

Tissue sampling and processing

Promptly or $24 \mathrm{~h}$ after exercise the TA muscles were removed bilaterally and rapidly freeze-clamped using a pair of liquid nitrogen cooled metal tongs. In the samples taken acutely after exercise, the time interval between the final contraction and freezing the exercised muscles was standardized at $30 \mathrm{~s}$, while time between cutting and freezing was maintained at $5 \mathrm{~s}$. The mid-belly region of the muscle samples taken $24 \mathrm{~h}$ after exercise was snap frozen in melting isopentane for histological examination. Twenty serial transverse cryosections of $10 \mu \mathrm{m}$ were cut at $-20^{\circ} \mathrm{C}$ (Anglia Scientific), routinely stained with hematoxylin-eosin to study histologicall features of injury and were examined microscopically for inflammatory changes. To obtain representative information about the occurrence of damage in the mid-belly region of the examined muscle (29), every second section was examined. Muscle fibers were considered to be damaged when they were infiltrated with mononuclear cells (2), had multiple central nuclei (13) or had a swollen appearance (24).

For biochemical analyses, frozen muscle tissue was ground with a mortar and pestle cooled with liquid nitrogen. Tissue was stored at $-80^{\circ} \mathrm{C}$ until analysis. Prior to analysis, $\sim 200 \mathrm{mg}$ wet weight (ww) of this deeply frozen, grounded muscle mixture was freeze-dried overnight at $-30^{\circ} \mathrm{C}$ for analysis of high-energy phosphates and related compounds as well as for analysis of muscle glycogen. Of the remainder, $\sim 50 \mathrm{mg}$ ( $(w w)$ was used for preparation of a muscle homogenate (50 $\mathrm{mg}$ $(w w) / \mathrm{ml}$ ) in a SET buffer (sucrose $0.25 \mathrm{M}$, EDTA $2.0 \mathrm{mM}$. TRIS $10.0 \mathrm{mM})(\mathrm{pH} 7.4$ ) to assess activity of glycogen synthase (GS) and cytochrome $C$ oxidase (CytCox). Adenine nucleotides and related compounds were assessed with a HPLC method as described by Wijnants and Van Belle (35) and modified by Van der Vusse et al. (31). Total adenine nucleotide content (TAN) was computed as the sum of ATP, ADP and AMP. Glycogen, glucose and phosphocreatine (PC) were assessed as described earlier $(30,31)$. The values, obtained by fluorometrically measuring the glucose residues, were corrected for free glucose present at the time of tissue sampling. Values were expressed as $\mu \mathrm{mol} \cdot \mathrm{min}^{-1} \cdot \mathrm{g}^{-1}$ dry weight (dw).

GS activity was assessed in the muscle homogenate at $30^{\circ} \mathrm{C}$. To discriminate between the active (GSi) part of GS and total GS activity (GSi+d), GSi activity was assessed in absence of glucose-6-phosphate (G6P) while G6P (10 $\mathrm{mM}$ ) was added to the homogenate to assess total GS activity. UDP-glucose was used as starting reagents and formation of UDP was measured according to Danforth (7). In the same homogenate, activity of CytCox was assessed at $30^{\circ} \mathrm{C}$ according to Gohil et al. (11). Enzyme activities were analyzed on a centrifugal analyzer (Cobas Fara, Hoffman La-Roche, Switzerland) and expressed as $\mu \mathrm{mol} \cdot \mathrm{min}^{-1} \cdot \mathrm{g}^{-1}$ wet weight $(w w)$. 


\section{Statistics}

Probably because of the low number of replicates, some of the data lested did nat show a normal distribution. Therefore we have chosen to perform non-parametric testing on all parameters. Analysis of data on consecutive time points of both the lengthening and the isometric exercised group was performed using the nonparametric Kruskal-Wallis rank test. To compare exercised muscles with nonexercised muscles (paired comparisons) the non-parametric Wilcoxon signed rank test was used. For unpaired comparison of data between different animals (testing differences between lengthening and isometric exercise) the Mann-Whitney $U$ test was used. For all comparisons the level of significance was set at $P<0.05$. Data are presented as mean with standard errors of the mean (SEM).

\section{Results}

The exercise protocol used in the present study is identical to the one used previously (12) where no inflammatory response could be detected $24 \mathrm{~h}$ after 60 LC (Fig 4.1a) or after 60 IC (data not shown). In the present study, examination of muscles dissected $24 \mathrm{~h}$ post-exercise revealed that muscles subjected to $240 \mathrm{IC}$ showed no histological signs of muscle damage (Fig 4.1 b) while after 240 LC distinct signs of muscle injury were present (Fig 4.1c). This is in line with earlier observations when using an identical protocol (12).
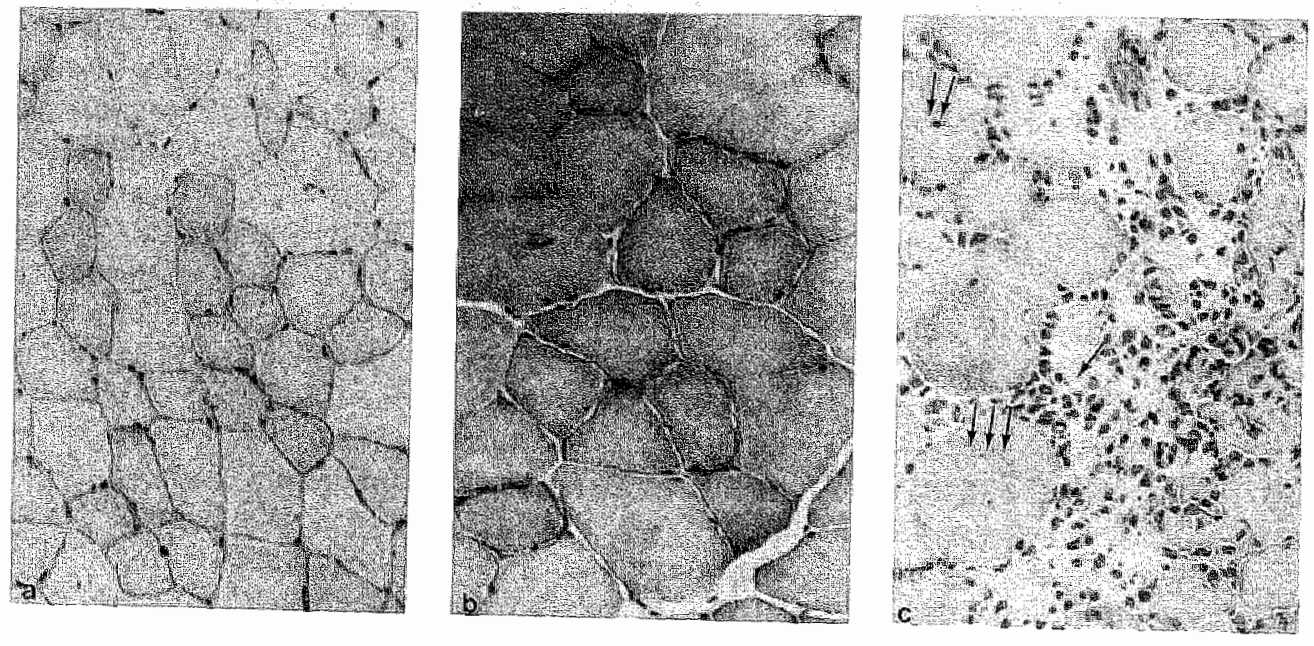

Figure 4. 1 : Representative phatomicrographs of cross sections of the mid-belly region of TA muscle $24 \mathrm{~h}$ after 60 forced lengthening contractions (a) (data from previous work (12)), $24 \mathrm{~h}$ after 240 isometric contractions (b) or $24 \mathrm{~h}$ after 240 lengthening contractions (c). Arrows point towards infiltrated mononuclear cells $(\mathbf{+})$, multiple central nuclei $(\mathbf{+})$ and swollen muscle cells $(\mathbf{+}+\mathbf{t}$.

Despite standardized freezing rates ( $30 \mathrm{~s}$ after termination of exercise), we observed a wide and unexplained range in phosphocreatine (PC) values in control muscles. Control values of other metabolites assessed in the same muscle homogenate (ATP, ADP, AMP. IMP, and glycogen) did not show this much 
variation. We failed to detect systematic differences between groups when comparing the pattern of variation in PC with the control values of other metabolites. Phosphocreatine decreased significantly in muscle samples taken immediately after $60 \mathrm{LC}$ and $60 \mathrm{IC}$. However, no decline was observed after 240 contractions compared to control muscles, independent of the type of exercise. Twenty-four hours after 240 IC tissue PC levels were increased, compared to control muscles (Table 4.1).

Mean muscle ATP content immediately post-exercise was not significantly affected by 60 LC or IC (Table 4.1), while immediately after 240 LC and 240 IC muscle ATP content had significantly declined compared to control muscles. ATP levels did not return to control values within $24 \mathrm{~h}$ after $240 \mathrm{LC}$, while ATP content $24 \mathrm{~h}$ after 240 IC was restored (Table 4.1). Muscle ATP content immediately after 240 LC was significantly lower than after the same number of isometric contractions.

No changes in ADP and AMP levels were detected (data not shown). When muscle contents of ATP, ADP and AMP are added up and individual differences between the exercised and non-exercised muscles are computed (TAN, total adenine nucleotides), it appears that after 60 LC TAN declined significantly compared to $60 \mathrm{IC}$ (Table 4.2). After 240 contractions TAN declined significantly after both types of exercise, TAN had fallen significantly more after 240 LC than after 240 IC. Twenty-four hours after LC, TAN was still depressed while after 240 IC it had returned to control values.

Individual differences in inosine monophosphate (IMP) content between exercised and non-exercised muscles responded inversely proportional to TAN except for muscles sampled 24 th after LC when TAN was still depressed while IMP had returned to control values (Table 4.2).

The levels of the degradation products of IMP (xanthine and hypoxanthine) did not exceed the lower detection limit of the HPLC method used (data not shown).

\begin{tabular}{|c|c|c|c|c|c|c|}
\hline \multirow[b]{3}{*}{ Isometric contractio } & \multicolumn{2}{|c|}{$\begin{array}{c}\text { ATP } \\
\text { (umol*g" dry weight) }\end{array}$} & \multicolumn{2}{|c|}{$\begin{array}{c}P C \\
\text { (umolmg" dry weighi) }\end{array}$} & \multicolumn{2}{|c|}{$\begin{array}{c}\text { CytCox } \\
\text { (umol-min "og wel welght) }\end{array}$} \\
\hline & Conturots & Exercise & Controls & Exerclse & Controls & Exercise \\
\hline & & & & & & \\
\hline $60 \mathrm{IC}$ & $35.7(0.7)$ & $32.6(1.6)$ & $90.2(5.3)$ & $61.6(7.1)^{*}$ & $15.5(1.7)$ & $15.5(1.0)$ \\
\hline $2401 \mathrm{C}$ & $35.9(1.2)$ & $30.5(1.2)^{* \prime}$ & $84.7(6.4)$ & $89.6(4.2)$ & $18.8(1.4)$ & $14.1(1.5)^{*}$ \\
\hline $24 \mathrm{~h}$ after $240 \mathrm{lC}$ & $35.4(0.6)$ & $33.1(1,1)$ & $70.0(6.0)$ & $96.3(3.5)^{*}$ & $19.7(2.02)$ & $15.7(0.8)$ \\
\hline \multicolumn{7}{|c|}{ Lengtheming contractions } \\
\hline $60 \mathrm{LC}$ & $33.5(1.0)$ & $30.0(0.7)$ & $82.5(4.0)$ & $61.6(7.1)^{*}$ & $15,0(1.7)$ & $17.8(0.9)$ \\
\hline $240 \mathrm{LC}$ & $32.0(2.2)$ & $25.5(1.0)^{*} \#$ & $62.7(6.3)$ & $77.9(6.8)$ & $15.5(0.8)$ & $15.5(1.5)$ \\
\hline $24 \mathrm{~h}$ after $240 \mathrm{LC}$ & $37.7(0.3)$ & $30.7(1.2)^{*}$ & $74.7(6.5)$ & $75.8(8.8)$ & $16.5(2.0)$ & $12.1(1.2)^{*}$ \\
\hline
\end{tabular}

Table 4.1: ATP and PC content of rat TA muscle ( $\mu \mathrm{mol}^{-\mathrm{g}^{-1}} \mathrm{dry}$ weight $)$ and cytochrome $\mathrm{C}$ oxidase (CytCox) activity ( $\mu$ moll-min ${ }^{-1} \cdot \mathrm{g}^{-1}$ wet weight). Data are presented as mean with SEM $(n=6) .{ }^{*}$ denotes a significant difference between exercised and non-exercised muscles. \# denotes a significant difference compared to isometrically exercised muscles. 


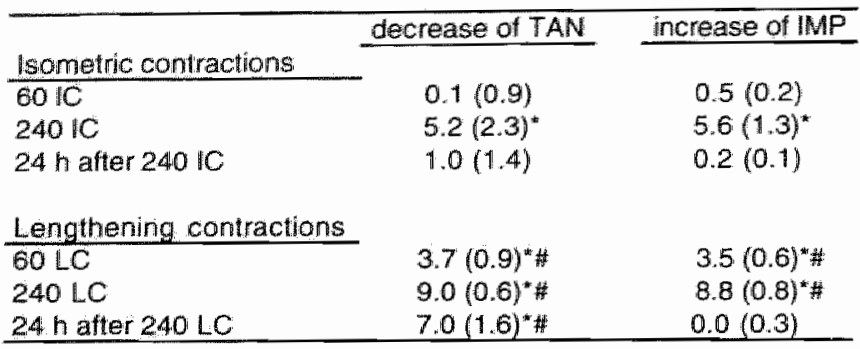

Table 4.2: Mean differences between the exercised muscle and the corresponding non-exercised control of total adenine nucleotide (TAN) and IMP content ( $\mu$ mol. $\mathrm{g}^{-1} \mathrm{dry}$ weight). Data are presented as mean with SEM ( $n=6)$. * denotes a significant post-exercise change with respect to non-exercised muscles, \# denotes a significant difference compared to isometrically exercised muscles.
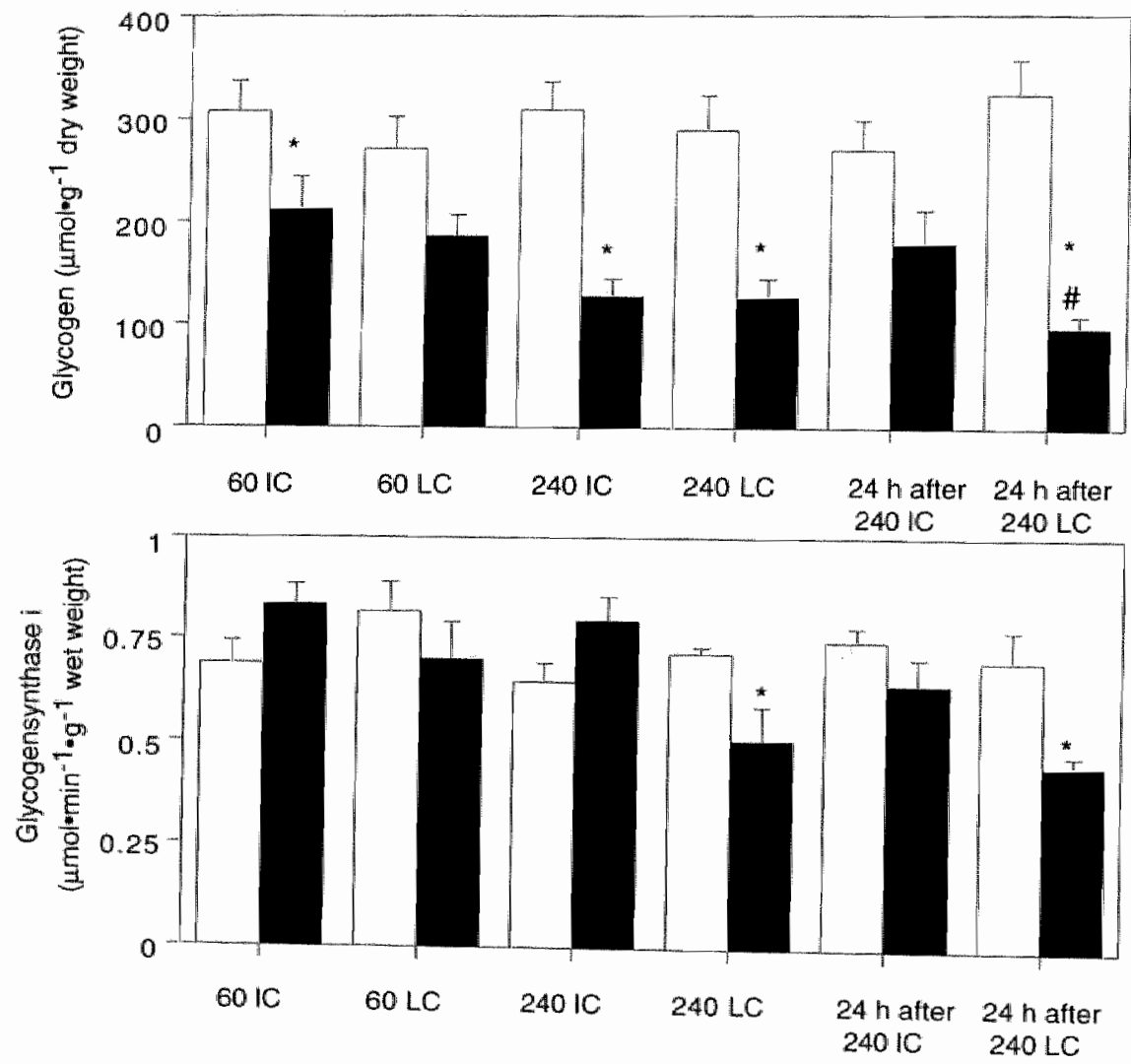

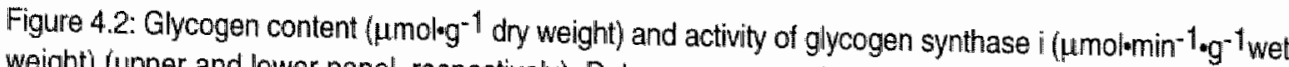
weight) (upper and lower panel, respectively). Data are presented as mean with SEM $(n=6) .1 C$ and LC. refer to isometric and lengthening contractions, respectively. " denotes a significant difference between control muscles (open bars) and exercised muscles (black bars). denotes a significant difference compared to isometrically exercised muscles. 
Glycogen content of the exercised muscles after $601 \mathrm{C}$ as well as immediately after 240 LC and 240 IC was significantly lower than in contralateral controll muscles. A near significant ( $P=0.074$ ) decline was observed directly after 60 LC (Figure 4.2 , upper pane). Muscles subjected to lengthening exercise failed to replenish muscle glycogen during the $24 \mathrm{~h}$ post-exercise period, in contrast to the observed increase in muscie glycogen $24 \mathrm{~h}$ after $240 \mathrm{lC}$. Muscle glycogen 24 th after $240 \mathrm{LC}$ was lower than $24 \mathrm{~h}$ after 240 IC as well as lower than muscle glyoogen in corresponding controls.

Directly and $24 \mathrm{~h}$ after lengthening exercise the active form of glycogen synthase (GSi) was lower than control values (Figure 4.2, lower panel), while no changes were observed in total glycogen synthase activity (data not shown).

The activity of CytCox in TA muscle, used as a marker enzyme for mitochondrial density, was declined $24 \mathrm{~h}$ after $240 \mathrm{LC}$ when compared to the corresponding control muscle as well as compared to $24 \mathrm{~h}$ after 240 /C (Table 4.1). After 240 isometric contractions CytCox activity was lower in exercised muscles than in control muscles.

\section{Discussion}

Examination of cross-sections of muscles dissected $24 \mathrm{~h}$ post-exercise revealed that 240 LC caused damage to the exercised muscles whereas isometric contractions (upto 240) can be considered as non-damaging exercise. The type and extent of muscle damage was similar to previous observations using identical experimental conditions (12). Therefore, force development after successive isometric and lengtheming contractions can reasonable be assumed to be of the same order of magnitude as observed previously (12).

During voluntary activity, fewer motor units are recruited while lengthening a muscle than during shortening or isometric contractions and less ATP is needed to develop the same force (1). During lengthening contractions each $300 \mathrm{~ms}$ contraction comprised $250 \mathrm{~ms}$ of isometric contraction and $50 \mathrm{~ms}$ of lengthening. isometric exercise consisted of $300 \mathrm{~ms}$ isometric contraction. Since lengthening lasted only a small fraction of the time and contractile velocity was relatively low $\left(1000^{\circ} \cdot \mathrm{s}^{-1}\right)$, it is likely that the metabolic demand in the lengthening and isometric exercise bouts is similar. Electromyography studies of rat hindlimb muscle during normal locomotion revealed that during intermediate locomotion (step cycle of 310 $\mathrm{ms}$ ) the tibialis anterior muscle contracted for $146 \mathrm{~ms}(21)$. In the present study, both contractile duration and the time span between successive contractions was longer than during normal locomotion. Despite the discrepancy between normal locomotion and the protocol chosen in the present study the amount and extent of muscle damage resembles observations in rats following downhill running (2).

\section{Acute effects}

Our contention that the metabolic demand of both types of exercise used in our model (lengthening and isometric) is comparable, is supported by similar glycogen content after $60 / C$ and $L C$ as well as after 240 IC and LC.

The decline in TAN $\left(3.7 \mu \mathrm{mol} \cdot \mathrm{g}^{-1} \mathrm{dw}\right)$ after $60 \mathrm{LC}$ was approximately matched by increased IMP $\left(3.5 \mu\right.$ mol $\left.\cdot \mathrm{g}^{-1} \mathrm{dw}\right)$, indicating a metabolic imbalance. When increasing the number of contractions, TAN declined with $5.2 \mu \mathrm{mol} \cdot \mathrm{g}^{-1} \mathrm{dw}$ 
after 240 IC while after $240 \mathrm{LC}$ a decline of $8.9 \mu \mathrm{mol} \cdot \mathrm{g}^{-1} \mathrm{dw}$ was observed. Again the decline in TAN was matched by increased IMP content (5.6 and $8.8 \mu \mathrm{mol} \cdot \mathrm{g}^{-1}$ $\mathrm{dW}$ after $240 \mathrm{IC}$ and $240 \mathrm{LC}$ respectively). The difference in tissue IMP content between isometrically and eccentrically exercised muscles was independent on the number of contractions ( 3.0 and $3.2 \mu \mathrm{mol}^{-1} \mathrm{~g}^{-1} \mathrm{dw}$ after 60 and 240 contractions, respectively). The observation that lengthening exercise induces an acute rise in IMP that is significantly higher than following isometric exercise is in correspondence with earlier findings (28). However, an explanation for this intriguing finding is still lacking. Remarkably, previous reports on post-exercise muscle force after isometric and eccentric exercise revealed that post-exercise muscle force after $60 \mathrm{IC}$ possessed a $26.3 \%$ difference compared to LC. Despite a 4-fold increase in the number of contractions the force deficit between IC and LC remained similar (25.8\% after 240 contractions (12)).

Enhanced tissue IMP content, accompanied by an increase in $\mathrm{NH}_{3}$ (not analyzed in the present study), correlate highly with fatigue (10) and may depress muscle contractility. IMP-induced inhibition of the actin-activated $\mathrm{Mg}^{2+}$-myosin ATP-ase activity thas been reported in vitro (33) thus preventing the formation of the actin-myosin complex, leading to decreased contractile performance of the muscle fibers. When extrapolation of findings of Westra et al. (33) to the present experimental set-up is allowed, an attractive mechanistic explanation is offered for the previously reported profound decline in muscle contractility after damaging lengthening exercise (12). Combination of the observation that both differences in IMP content after IC and LC and the post-exercise force deficit between IC and LC are independent on the number of contractions points towards a major role of IMP in muscle contractile malfunction after lengthening exercise.

\section{Sustained effects}

Twenty-four hours after exercise, ATP content and TAN in isometrically exercised muscles returned to control values. In contrast, muscles subjected to $240 \mathrm{LC}$ still had depressed ATP and TAN levels while muscle IMP returned to control values after both types of exercise. This indicates that during periods of muscle damage induced by lengthening exercise, IMP is dephosphorylated via 5'nucleotidase to inosine and inorganic phosphate rather than used for resynthesis of high-energy phosphates after conversion to AMP in the purine nucleotide cycle. Oxidation of inosine results in hypoxanthine which can irreversibly be oxidized to xanthine and uric acid (27). HPLC analysis showed no elevation of any of these purines in the exercised muscles. Hypoxanthine can easily pass through cellular membranes, and can be irreversibly converted into xanthine and uric acid, this may induce a net loss of adenine nucleotides from the damaged muscles.

Sustained decline in TAN in damaged muscles may also be due to a lower number of intact mitochondria, the major site of ATP regeneration. Loss of sarcolemma and mitochondrial membrane integrity is a common finding after damaging eccentric exercise (for review see 3) and may on the one hand result in a depressed capacity to regenerate ATP and on the other hand may permit release of (a part of) the adenine nucleotides into the extracellular compartment. The observed decline in activity of CytCox may point towards a lower number of intact mitochondria and therefore favors this hypothesis. 
After lengthening exercise, muscle glycogen was declined for at least $24 \mathrm{~h}$. This finding is in line with earlier observations $(6,8,14,22,28,34)$. The lack of recovery of muscle glycogen can be caused by a decreased resynthesis, an increase in use, or a combination of both. Costill et al. (6) speculated that inflammatory cells infiltrating traumatized muscle tissue elevate glucose oxidation. In the same study, examination of glycogen synthase activity revealed that directly after lengthening exercise the percentage of this enzyme in its active form was lower than after concentric exercise (6). Glycogen synthase activity in Costill's study may have been biased by different post-exercise glycogen levels after concentric versus eccentric work. In an elegantly designed study by Doyle et al. (8), where muscle glycogen stores were equally depleted after eccentric and concentric work, glycogen synthase activity declined non-significantly after eccentric work. In the present study we conclusively demonstrate that the total activity of glycogen synthase remained unchanged while glycogen synthase in its active form was depressed immediately, as well as $24 \mathrm{~h}$ after damaging (lengthening) exercise. Muscle glycogen content immediately after lengthening and isometric exercise was comparable, therefore, the lower activity of GSi is not clouded by different post-exercise muscle glycogen levels. Depressed activity of GSi may account for the lack of glycogen repletion during a $24 \mathrm{~h}$ time interval after extensive lengthening work. It can, however, not be excluded that other factors, such as an increase in glucose oxidation by inflammatory cells or a decreased glucose uptake in the affected muscle cells, contribute as well.

In summary, it is shown that muscle glycogen declined similarly after isometric and lengthening contractions, indicating a comparable metabolic demand of LC and IC, if performed corresponding to the present study. However, decreased TAN content and increased IMP levels after exercise both point towards an acute deterioration in adenine nucleotide metabolism. This metabolic deterioration is more severe after lengthening exercise than after isometric exercise and may contribute to muscle contractile malfunction after lengthening exercise. Twenty-four hours after lengthening exercise muscle glycogen, total adenine nucleotides (TAN) and the activity of GSi and CytCox were still depressed, reflecting a sustained deterioration of energy metabolism in the affected muscles during the $24 \mathrm{~h}$ recovery period. The failure to restore muscle glycogen stores to pre-exercise levels within $24 \mathrm{~h}$ after damaging exercise is most likely caused by a permanently depressed glycogen synthase (GSi) activity in muscles subjected to $240 \mathrm{LC}$ during the $24 \mathrm{~h}$ recovery period, as observed in the present study. Since sustained metabolic deterioration was observed after damaging exercise only, it is concluded that, despite a comparable metabolic demand, damaging lengthening exercise hampers formation of high-energy phosphates for at least $24 \mathrm{~h}$, while nondamaging isometric exercise allows for complete restoration of energy metabolism. 


\section{References}

1. Abbott, B. C., B. Bigland, and J. M. Fitchis. The physiological cost of negative work. I Physiol 117:380300,1952

2. Armstrong, R. B., R. W. Oglivie, and J. A. Schwane. Eccentric exercise induced injury to skeletal muscie. $d$ Appl Physiol 54: 80-93, 1983.

3. Armstrong, R. B., G. L. Warren, and J. A. Warren. Mechanisms of exercise-induced muscle fiber injury. Sports Med 12: 184-207, 1991.

4. Clarkson, K. Nosaka, and B. Braun. Muscle function after exercise-induced muscle damage and rapid adaptation. Med Sci Sports Exerc 24: 512-520, 1992.

5. Cleak, M. J., and R. G. Eslon. Muscle soreness, swelling, stiffness and strength loss atter eccentric exercise, Br J Sp Med 26: 267-272, 1992

6. Costill, D. L., D. D. Pascoe, W. U. Fink, A. A. Robergs, S. I. Barr, and D. Pearson. Impaired muscle glycogen resynthesis after eccentric exercise. I Appl Physio' 69: $46-50,4990$.

7. Danforth, W. H. Glycogen synthetase activity. Interconversion of two forms and control of glycogen synthesis. J Biol Chem 240: 588-593; 1965.

8. Doyle, J. A., W. M. Sherman, and R. L. Strauss. Elfects of eccentric and concentric exercise on muscle glycogen replenishment. J App/ Physio/ 74: 1848-1855, 1993.

9. Ferry, A., I. Amiridis, and M. Fiev. Glycogen repletion and synthesis in the rat after downhill running. Eur d Appl Physiol 64: 32.35, 1992.

10. Filts, R. H. Cellular mechanisms of muscle fatigue. Physiological reviews 74: 49-94, 1994.

11. Gohil, K., D. A. Jones, and R. H. T. Edwards. Analysis of muscle mitochondrial function with techniques applicable to needle biopsy samples. Clin Pinysiol 1: 195-207, 1981.

12. Hesselink, M. K. C. H. Kuipers, P. Geurten, and H. van Straaten. Structural muscle damage and muscle strength after incremental number of isometric and forced lengthening contractions. I Muscle Res cell Motil 17: 335-341, 1996.

13. Kuipers, H., J. Drukker, P. M. Frederik ${ }_{\text {. P. Geurten }}$ and $G$. vain Kranenburg. Muscle degeneration after exarcise in rats. Int J Sports Med 4: 49-55, 1983.
14. Kuipers, H. H. A. Keizer, F. T.J.H. Verstappen, and D. L. Costifl. Influence of a prostaglandin-inhibiting drug an muscle soreness after eccentric work. int $J$ Sponts Med 6: 336-339, 1985.

15. Lieber, R. L., and J. Friden. Muscle damage is not a function of muscle force but active muscle strain. I Appt Physial 74: 520-6, 1993 .

16. Lieber, R. L., T. M. Woodburn, and J. Friden. Muscle damage induced by eccentric contractions of 25\% strain. J Appl Physiol 70: 2498-507, 1991.

17. Lowe, D. A., G. L. Warren, C. P. Ingalls, D. B. Boorstein, and R. B. Armstrong. Muscle function and protein metabolism after initiation of eccentric contraction-induced injury. $J$ Appl Physiol 79: 1260-1270, 1995.

18. McCully, K. K, Z. Argov, B. P. Boden, R. L. Brown, W. J. Bank, and B. Chance. Detection of muscle injury in humans with $31 . p$ magnetic resonance spectroscopy. Muscle \& Nerve 11:212-216, 1988.

19. McCully, K. K., and J. A. Faullkner. Characteristics of lengthening contractions associated with injury to skeletal muscle ribers. J App/ Physiol 61: 293-299, 1986.

20. Newham, D. J., D. A. Jones, and P. M. Clarkson. Repeated high-force eccentric exercise: effects on muscle pain and darnage. $/$ Appl Physiol 63: 1381-6. 1987.

21. Nicolopoulos-Stournaras, S., and J. F. lles. Hindlimb muscle activity during bocomation in the rat. $J$ Zool London 203: 427-440, 1984

22. OReilly, K. P. M. J. Warhol, A. A. Fielding, W. R. Frontera, C. N. Meredith "and W. J. Evans. Eccentric exercise-induced muscle damage impairs muscle glycogen repletion. J App' Physiol 63: 252-256, 1987.

23. Pascoe, D. D., D. L. Costill, W. J. Fink, R. A. Robergs, and J. J. Zachwieja. Glycogen resynthesis in skeletall muscle following resistive exercise. Med Sci Sponts Exerc 25: 349-354, 1993

24. Peeze-Binkhorst, F. M., D. W. Slaaf, H. Kuipers, G. J. Tangelder, and $\mathrm{B}$. S. Renernan. Exercise-induced swelling of rat soleus muscle: its relationship with intramuscular pressure. $J$ Appl Physiol 69: 67-73, 1990

25. Rodenburg, J. B., P. R. Bar, and B. R. De. Relations between muscle soreness and biochemical and 
functional outcomes of eccentric exercise. I Appl Physiol 74: 2976-83, 1993.

26. Teague, B. NN., and J. A. Schwane. Effect of intermittent eccentric contractions on symptoms muscle microinjy. Med Sci Sports Exerc 27: 1378-1384, 1995.

27. Tullson, P. $C_{n}$, and R. L. Teriung. Adenine nucleotide degeneration in striated muscle. Int $J$ Sponts Med 11: S47-S55, 1990.

28. van der Meulen, J. H. H. Kuipers, F. R. M. Stassen, H. A. Keizer, and G. J. van der Vusse. High energy phosphates and related compounds, glycogen levels and histology in the rat tibialis anterior muscle after forced lengthening and isometric exercise. Plluggers Arch 420: 354:358, 1992.

29. van der Meulen, J. H., H. Kuipers, J. C. van derWal, and J. Drukker. Quantitative and spatial aspects of degenerative changes in rat soleus muscle after exercise of different durations. J Anat 182: 349-353, 1993.

30. van der 'Vusse, $G_{n} J_{1}, W$. A. Coumans, F. H. wan der Veen, A. Drake, W. Flameng, and R. Suy. ATP, creatine phosphate and glycogen content in human myocardial biopsies: markers for the efficacy of cardiopratection. Vasc Surg 8: 127-134, 1984.

31. van der Vusse, G. d., M. van Bilsen, and R. S. Reneman. Is phospholipid degradation a critical event in ischemia and reperfusion damage? News Physiol Sci 4: 49-53, 1989 .

32. Warren, G. L., D. Hayes, D. A. Lowe, J. H. Williams, and R. B. Armstrong. Eccentric contraction-induced injury in normal and hindlimb-suspended mouse soleus and EDL muscles. J Appl Physioi 77: 1421-1430, 1964.

33. Westra, H. G., J. A. Berden, and W. J. Pasman. A model for the regulation of actin activated $\mathrm{Mg}^{2+}$. myosin ATPase; inhibition of the formation of actinmyosin complex by IMP. Neuromuscular fatigue, edited by A. J. Sargeant and D. Kernell, Amsterdam. North Holland, p. 24-26, 1992.

34. Widrick, J.J., D. L. Costill, G. K. McConell, D. E. Anderson, D. R. Pearson, and لJ. J. Zachwieja. Time course of glycogen accumulation after eccentric exercise. J Appl Physiol 72: 1999-2004, 1992.

35. Wijnants, $J$, and $H$. van Belle. Single-run high performance chromatography of nucleotides, nucleosides and major purine bases and its application to different tissue extracts. Anal Biochem 144: 258-266, 1985. 



\section{Muscle Glycogen Following Lengthening and Shortening Muscle Contractions in Relation to Activity of Glycogen Regulatory Enzymes and Muscle GLUT4 Content}

M.K.C. Hesselink, H.A. Keizer, M.R. Drost, Y.F. de Jong, H. Kuipers

Submitted. 


\begin{abstract}
Muscle glycogen resynthesis after damaging lengthening exercise was investigated by studying muscle glycogen content, related enzymes and muscle GLUT4 content $0,6,24$ and $48 \mathrm{~h}$ following lengthening and shortening exercise. Lengthening exercise induced extensive muscle damage, in contrast to shortening exercise. Despite a similar glycogen depletion after both types of exercise we observed striking differences in glycogen resynthesis. Following shortening and lengthening exercise a rapid (within 6 h) glycogen restoration phase exists. Following shortening exercise glycogen remained stable after restoration to control values within $6 \mathrm{~h}$. The first $6 \mathrm{~h}$ after lengthening exercise, glycogen was resynthesized with a significantly lower rate than after shortening exercise. Consequently, glycogen content was significantly lower following lengthening than following shortening exercise. Beyond $6 \mathrm{~h}$ after lengthening exercise, glycogen declined again. It is concluded that following lengthening muscle glycogen restoration is bi-phasic. A novel observation is that the bi-phasic repletion pattern coincides with a bi-phasic response in muscle GLUT4 content, while fractional activity of glycogen synthase remained depressed the entire post-exercise period.
\end{abstract}




\section{Introduction}

In vigorously contracting muscle ATP resynthesis relies on breakdown of carbohydrates. Carbohydrates can be stored as glycogen in skeletal muscle as well as in the liver. The importance of muscle glycogen as a substrate during high. intensity exercise is evidenced by the observation that low muscle glycogen levels correlate highly with fatigue (12). Replenishment of muscle glycogen stores to or beyond pre-exercise levels usually occurs within $48 \mathrm{~h}$ after non-damaging exercise (18). Following non-damaging (shortening) exercise, glycogen replenishment starts with a rapid restoration phase during the first 4-6 h post-exercise, with glycogen synthesis rates of 5 to $8 \mu \mathrm{mol} \cdot \mathrm{g}^{-1} \mathrm{ww} \cdot \mathrm{h}^{-1}(19-21)$, followed by a slow recovery phase during which glycogen rises to or beyond control values within 48 h post-exercise. However, following exercise-induced muscle damage, declined muscle glycogen levells are reported upto $72 \mathrm{~h}$ post-exercise $(7,11,29,30)$.

Blood-borne glucose is the major substrate for glycogen resynthesis. Consequently, glucose uptake from the blood is of paramount importance to replenish muscle glycogen stores. The importance of transport of glucose across the sarcolemma is emphasized by the observation that sarcolemmal transport of glucose is rate-limiting for glucose utilization $(13,32)$. Glucose transport across the sarcolemma is facilitated by 2 distinct glucose transporter proteins (GLUT1 and GLUT4). Basal transport of glucose is controlled by GLUT1 while GLUT4 is an insulin and contraction regulatable glucose transporter (e.g., 14).

In a series of recent papers Asp and co-workers attempted to elucidate the mechanilsm behind delayed glycogen repletion following damaging lengthening exercise $(2-5,22)$. Following lengthening exercise, muscle GLUT4 content was transiently decreased in humans $(2,3)$ as well as in rats $(4,22)$. Muscle GLUT4 content significantly declined 24 and 48 hours post-exercise after which it returned to control values within 4 days (3). However, no reports exist on muscle GLUT4 content during the first few hours after exercise-induced muscle damage and data on a rapid glycogen repletion phase after lengthening exercise are scarce.

To reproduce and extend the findings on declined GLUT4 content after exercise-induced muscle damage by Asp et al. (2-5), we studied muscle glycogen resynthesis during 48 hours following damaging llengthening and non-damaging shortening exercise in rats. Special attention was paid to the rapid phase of postexercise glycogen resynthesis $6 \mathrm{~h}$ after exercise by monitoring muscle glycogen content, the activity of glycogen regulatory enzymes and muscle GLUT4 content in rat tibialis anterior (TA) muscle after non-damaging (shortening) and damaging (lengthening) exercise. For comparison with earlier reports $(2-5)$, these parameters were also studied 0,24 and $48 \mathrm{~h}$ after both types of exercise.

\section{Materials and methods}

\section{Animals}

Twelve week old male Wistar rats (Charles River) were used in the present study. Rats were housed under standardized conditions ( $12 \mathrm{~h}$ dark/light cycle, $21-22^{\circ} \mathrm{C}$ and 50-60\% humidity). Exercise and muscle dissection were performed under pentobarbital anesthesia (Narcovet ${ }^{(1)} 1.0 \mathrm{ml}^{\circ} \mathrm{kg}^{-1}$ body weight, i.p. injection) upon short-term (less than $20 \mathrm{~s}$ ) $\mathrm{CO}_{2}$ sedation. After muscle dissection, rats were 
sacrificed by cervical dislocation. All experimental procedures were approved by the Institutional Animal Care and Use Committee of the Maastrich University and complied with the principles of laboratory animal care.

\section{Diet}

Prior to the experiment rats had free access to tap water and pelleted lab chow (SRM-A, Hope Farms, Woerden, the Netherlands) containing approximately 54\% carbohydrates, $28 \%$ protein, $7 \%$ fat, $7 \%$ minerals and $4 \%$ fibers ( $w / w)$. To prevent bias by diurnal variation in muscle glycogen stores, experiments were started at the same clock-time (8.00 am). Since a bolus injection of pentobarbital was used, we were unable to standardize the time span of recovering consciousness. To prevent differences in food intake induced by the duration of post-exercise consciousness, rats were deprived from food during $6 \mathrm{~h}$ post-exercise, with water supplied ad libitum.

\section{Exercise protocol}

Forty-eight rats were randomly assigned to one of 2 exercise regimes of either 240 shortening or lengthening contractions using a custom built rat dynamometer (RDM). During both exercise protocals the rat hindlimb dorsiflexor muscles (TA and EDL) were unilaterally subjected to 4 consecutive exercise bouts of 60 isokinetic $\left.\left(500^{\circ} \mathrm{ms}-1\right), 1.4 \mathrm{Lf} \cdot \mathrm{s}^{-1}\right)$ fused tetanic contractions. Dorsiflexor muscles were forced to contract by electrical stimulation via percutaneous needle electrodes placed close to the common peroneal nerve. Electrical stimulation was employed every 3 seconds for $310 \mathrm{~ms}$ using individually determined supramaximal stimulation conditions (typically $\sim 3 \mathrm{~V}, \sim 130 \mathrm{~Hz}$ ), sequential exercise bouts were interspaced by 5 minutes recovery.

The dynamometer used in the present study permits isokinetic dorsi-and plantarflexion in the tibiotarsal joint and is described in detail elsewhere (16). Briefly, an anaesthetized rat is placed on a platform in supine position. After positioning the rat, the foot is secured to a footplate with a knee angle of $\sim 90^{\circ}$. The footplate is secured to an axis inducing rotation in the tibiotarsal joint. Isokinetic rotation of the footplate around the anatomical position of the ankle can be performed over a range of $-40^{\circ}$ to $+40^{\circ}$ relative to the anatomical position. To ensure maximal muscle tension while lengthening or shortening the muscles, rotation is initiated $200 \mathrm{~ms}$ after the onset of electrical stimulation. Shortening and lengthening of the dorsiflexor muscles was performed around the optimal muscle length (at $-5^{\circ}$ tibiotarsal angle).

Muscle sampling and processing

Following both types of exercise. TA muscles were dissected bilaterally $0,6,24$ and $48 \mathrm{~h}$ post-exercise ( $n=6$ in every group). For histological examination the midbelly region of the TA muscle was used, mounted on a thin piece of cork with O.C.T. embedding compound (Tissue-tek ${ }^{\circ}$, Miles Laboratories) and frozen in isopentane cooled to melting point with liquid nitrogen. Cryosections of $10 \mu \mathrm{m}$ were cut at $-20^{\circ} \mathrm{C}$, thaw-mounted on glass slides and stained with hematoxylin and eosin for quallitative assessment of muscle damage by light microscopical examination. The remainder of the muscle was carefully dissected free from visible blood and connective tissue, ground with a liquid nitrogen cooled mortar and pestle and rapidly frozen. Tissue was stored at $-80^{\circ} \mathrm{C}$ until analysis. 
Prior to analysis, part of this grounded muscle mixture was used for preparation of a muscle homogenate $(50 \mathrm{mg} / \mathrm{ml}$ ) in a SET buffer (sucrose $0.25 \mathrm{M}$; EDTA $2.0 \mathrm{mM}$. Tris $10.0 \mathrm{mM})(\mathrm{pH} 7.4)$ to assess activity of glycogen synthase (GS) and glycogen phosphorylase (GP). The active part of glycogen synthase (GSi) was assessed in absence of glucose-6-phosphate (G6P) while G6P (10 mM) was added to the homogenate to assess total glycogen synthase (GSi+d) activity. UDP. glucose was used as starting reagent and formation of UDP was measured spectrophotometrically according to Danforth (8). Activity of glycogen phosphoryllase a (GPa) was assessed at $30^{\circ} \mathrm{C}$ in absence of AMP while total activity (GPa+b) was assessed by adding $5 \mathrm{mM}$ AMP to the homogenate. The formation of thexosephosphates was measured according to Harris et all. (15).

An other part of the muscle mixture was used to asses muscle glycogen content by dissolving muscle tissue for $1 \mathrm{~h}$ in $1 \mathrm{M} \mathrm{NaOH}\left(37^{\circ} \mathrm{C}\right)$, precipitating glycogen with $96 \%$ ethanol $110 \mathrm{~min}$ at $80^{\circ} \mathrm{C}$ followed by overnight precipitation at $4^{\circ} \mathrm{C}$ ). Subsequiently the pellet was hydrolyzed using $1 \mathrm{M} \mathrm{HCl}$ at $100^{\circ} \mathrm{C}$ for $3 \mathrm{~h}$ after which $\mathrm{HCl}$ was neutralized with a $\mathrm{KCl}$ saturated $\mathrm{KOH} / \mathrm{Tris}(2.1 \mathrm{M} / 0.12 \mathrm{M})$ buffer. Glycogen derived glycosyl units were dettermined using a glucose kit (hexolkinase method, Roche) for a centrifugal analyzer. Enzyme activities and muscle glycogen content were analyzed on a centrifugal analyzer (Cobas Fara, Hoffman La-Roche, Switzerland) enzyme activities were expressed as $\mu$ mol.min ${ }^{-1} \cdot g^{-1}$ wet weight (ww) as well as percentage fractional activity (GSi*GSi+d-1 and GPa*GPa+b-1). Glycogen was expressed as $\mu$ mol glycosyl units $\mathrm{g}^{-1} \mathrm{ww}$.

Total muscle GLUT4 content was quantified by Western blotting using an affinity purified polyclonal antibody raised in rabbits against the $15-C$ terminus of rat GLUT4 (kindly provided by Dr. A. Zorzano, Barcelona, Spain) In more detail, frozen muscle $(\sim 70 \mathrm{mg}$ ) was homogenized by ultra turrax in $1000 \mu l$ ice-cold buffer (50 mM Hepes, $10 \mathrm{mM}$ EDTA, $250 \mathrm{mM}$ sucrose, $10 \mathrm{mM}$ phenyl-methanesulphonyl fluoride (PMSF) and $400 \mathrm{U} / \mathrm{ml}$ Trasylol). After homagenizing the tissue, the homogenate was centrifuged $\left(4^{\circ} \mathrm{C}\right)$ for $15 \mathrm{~min}$ at $3000 \mathrm{~g}$. Subsequently total crude muscle membranes were obtained from the supernatant by 90 min ultracentrifugation at $40.000 \mathrm{rpm}$ using a Beckmann Ti70.1 rotor $\left(4^{\circ} \mathrm{C}\right)$. The pellet was resuspended in $100 \mu l$ phosphate-buffered saline (PBS, $10 \mathrm{mM}, \mathrm{pH} 7.4$ ), 50 l. was. used for determining total crude membrane protein content using the macrobichoninic acid method while the remainder was solubilized in $15 \mu$ distilled water and $10 \mu \mathrm{l}$ sample buffer $(50 \%$ distilled water $(\mathrm{v} / \mathrm{v}), 12.5 \% 0.5 \mathrm{M}$ Tris-HCl $(\mathrm{v} / \mathrm{v})(\mathrm{pH}$ 6.8), $10 \%$ glycerol $(\mathrm{w} / \mathrm{w}), 20 \%$ sodiumdodecylsulfate $(\mathrm{SDS}, 10 \%(\mathrm{w} / \mathrm{v})), 5 \%, 2$ mercapto-ethanol ( $v / v)$ and $2.5 \%$ bromphenol blue $(0.1 \% \mathrm{w} / \mathrm{v}))$. Using a $10 \%$ polyacrylamide gel (w/v), samples (4 $4 \mathrm{~g}$ protein) were subjected to SDS polyacrylamide gel electrophoresis (mini-protean II, Bio Rad) at $200 \mathrm{~V}$. Following electrophoresis, the proteins are electro-transferred to a nitrocellullose membrane at $100 \mathrm{~V}$ for $1 \mathrm{~h}$. Blocking was done by incubation for $1 \mathrm{~h}$ with $1 \%$ BSA (w/v) in PBS, subsequently the membrane was rinsed with PBS containing $0.5 \%$ Triton $X-100$ (PBS-T) followed by incubation with a polyclonal rabbit anti GLUT4 1 1:500 in PBST) at room temperature overnight. After incubation with the primary antibody the nitrocellulose membrane was rinsed 3 times in PBS-T followed by 60 min incubation with swine anti rabbit peroxidase (1:1000 in PBS-T) at room 
temperature. Finally, after washing the nitrocellulose membrane in PBS-T ( 3 times $5 \mathrm{~min}$ ), total GLUT4 protein content was visualized using an enhanced chemiluminesence kit (Amersham Life Science, UK) and quantified by computerized densitometry. Immunoblotting procedures were monitored by adding a mixed hindlimb muscle (triceps surae) preparation with known GLUT4 content on lane 1 of every blot as internal control. Total GLUT4 content of the non-exercised muscles was set at $100 \%$.

Statistics

Successive post-exercise sample points $(0,6,24$ and $48 \mathrm{~h})$ were compared by factorial ANOVA and differences, if any, were located using Scheffé post-hoc testing. Values of exercised and contralateral non-exercised muscles were compared by Student's paired t-test while samples taken after lengthening and shortening exercise were compared using unpaired Student's t-test. Unless stated otherwise, data are presented as mean $\#$ standard error of the mean (SEM), the levell of significance was set at $p<0.05$.

\section{Results}

Histological examination of muscles sampled $48 \mathrm{~h}$ post-exercise revealed that massive muscle damage, as indicated by multiple central nuclei, infiltration of phagocytic cells and fiber necrosis, was present following lengthening exercise while no such changes were observed after shortening exercise (Fig 5.1).
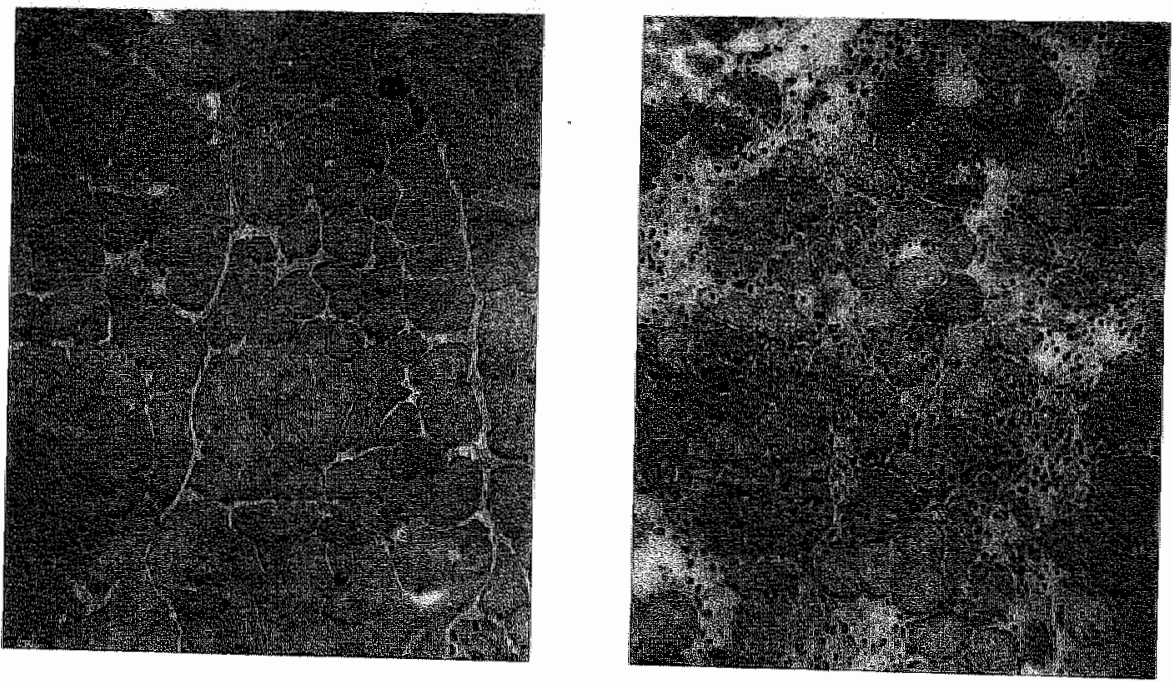

Figure 5.1: Representative photomicrographs of cross sections following shortening and lengthening exercise (left and right panel respectively). Note the presence of leukocytes and fiber necrasis in muscles subjected to lengthening contractions.

\section{Muscle gilycogen}

Muscle glycogen concentration declined similarly following lengthening and shortening exercise (Fig 5.2, upper panel). Six h after lengthening exercise, muscle glycogen returned to control values but was significantly lower than following 
shortening exercise. Within $6 \mathrm{~h}$ after shortening exercise, muscle glycogen returned beyond control values and remained stable 24 and $48 \mathrm{~h}$ post-exercise. Remarkably, after apparent restoration to control values within $6 \mathrm{~h}$ after lengthening exercise, 24 and $48 \mathrm{~h}$ post-exercise muscle glycogen was lower $(p<0.05)$ than nonexercised controls as well as after shortening exercise.
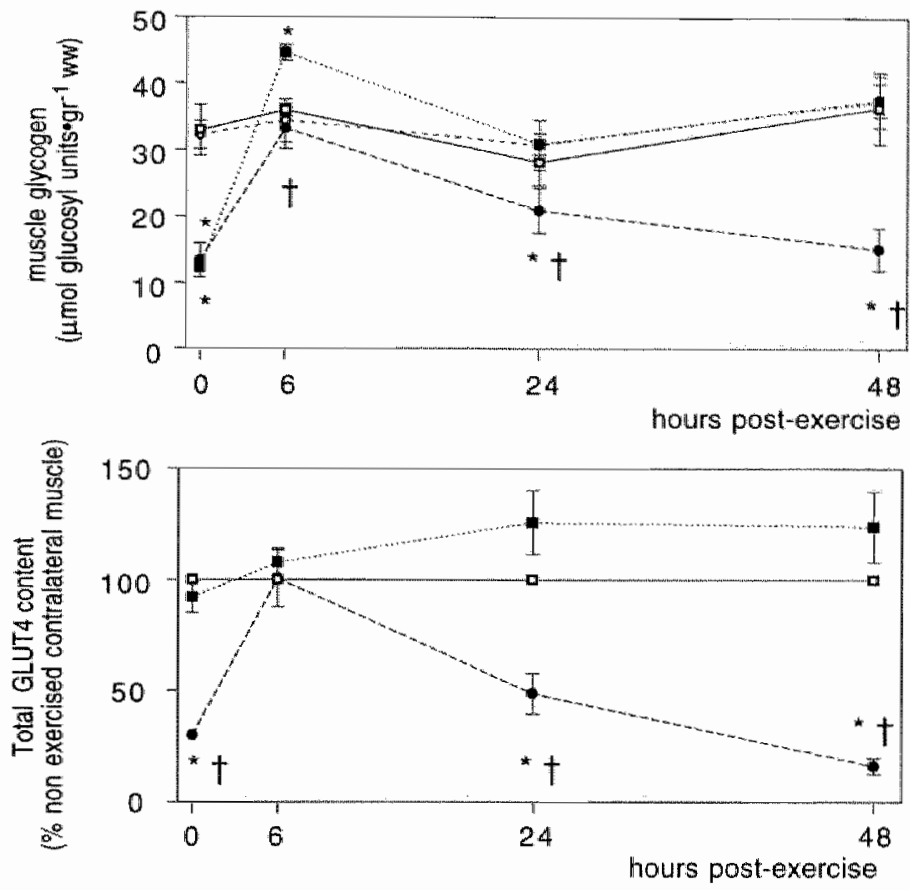

Figure 5.2: Total muscle glycogen content (upper panel) and muscle GLUT4 content (lower panel). Open and filled squares represent values from contralateral control muscles and muscles subjected to shortening exercise, respectively. Open and filled circles represent values from contralateral controll muscles and muscles subjected to lengthening exercise, respectively. * denotes a significant difference between exercised and non-exercised muscles, $\dagger$ denotes a significant difference between shortening and lengthening exercise. GLUT4 content in control muscles was set at 100\% for both types of exercise.

\section{Enzyme activity}

Total enzyme activity of GPa+b and GSi+d were not significantly affected by exercise (Table 5.1 and 5.2, respectively). Fractional activity of GP significantly increased immediately as well as $6 \mathrm{~h}$ after shortening exercise compared to nonexercised control values. Compared to lengthening exercise (Table 5.2), fractional activity of GP increased $6 \mathrm{~h}$ post-exercise. Twenty-four $\mathrm{h}$ after lengthening exercise, fractional activity was significantly elevated compared to 0,6 and $48 \mathrm{~h}$. Fractional activity of GS decreased significantly following lengthening exercise, compared to non-exercised controls at 6,24 and $48 \mathrm{~h}$ post-exercise as well as compared to shortening exercise at $0,6,24$ and $48 \mathrm{~h}$ post-exercise (Table 5.2). 


\begin{tabular}{|c|c|c|c|c|c|c|c|c|}
\hline & & ycogenphos & rylase lat & & Gycogen & hosph & Macliona & ictivity) \\
\hline & short & ening & Jenigth & gening & shor" & & & ning \\
\hline & exercise & controls & exercise & controlls & exercise & controls & exercise & controls \\
\hline & $14.31(0.97)$ & $1202(0.68)$ & $95.12(0.73)$ & $15.12(0.73)$ & $43.0(1.1)^{*}$ & $25.6(2,8)$ & $37.0(7.0)$ & $33.5(4.3)$ \\
\hline 6 & $12.75(0.82)$ & $15.65(1.72)$ & $15.19(0.92)$ & $15.19(0.92)$ & $37.9(4.0)^{\mathrm{A}} \dagger$ & $25.1(4.7)$ & $24.2(4.7)$ & $33.3(2.2)$ \\
\hline 24 & $12.50(0.83)$ & $12.88(1.38)$ & $14.37(1.25)$ & $14.37(1.25)$ & $34.4(6.2)$ & $26.8(3.9)$ & $42.7(5.9) \S$ & $30.8(3.1)$ \\
\hline 48 & $13.49(1.57)$ & $14.08(1,10)$ & $13.21(0.78)$ & $13.21(0.78)$ & $21.7(5.4)$ & $22.8(9.5)$ & $23.4(8.6)$ & $27.7(6.2)$ \\
\hline
\end{tabular}

Table 5.1: Total ( $\mu$ mol.min ${ }^{-1} \cdot \mathrm{g}^{-1}$ wet weight) and fractional activity (\%) of glycogen phosphorylase. * denotes a significant difference between exercised and non-exercised muscles, $t$ denotes a significant difference between shortening and lengthening exercise. $\$$ represents different from 0,6 and $48 \mathrm{~h}$ postexercise.

\begin{tabular}{|c|c|c|c|c|c|c|c|}
\hline \multicolumn{4}{|c|}{ Glycogensynthase $(i+d)$} & \multicolumn{4}{|c|}{ Glycogen synthase (fractional activity) } \\
\hline \multicolumn{2}{|c|}{ shortening } & \multicolumn{2}{|c|}{ lengthening } & \multicolumn{2}{|c|}{ shortening } & \multicolumn{2}{|c|}{ lengthening } \\
\hline exercise & controls & exercise & contrals & exercise & controls & exercise & controls \\
\hline $01.23(0.10)$ & $1.70(0.24)$ & $1.03(0.24)$ & $1.42(0.22)$ & $80.8(3.7)^{2}$ & $53.9(3.8)$ & $50.0(6.0) t$ & $61.1(4.0)$ \\
\hline $61.53(0.38)$ & $1.60(0.09)$ & $1.90(0.17)$ & $1.67(0.25)$ & $58.3(21)$ & $59.5(3.5)$ & $29.4(3.1)^{*} \#$ & $58.6(3.9)$ \\
\hline $24 \quad 1.24(0.07)$ & $1.26(0.13)$ & $1.38(0.081$ & $1.61(0.38)$ & $53.9(2.2)$ & $66.4(1.9)$ & $28.8(8.6)^{*}+1$ & $51.3(7.4)$ \\
\hline $48 \pi .61(0.30)$ & $1.21(0.13)$ & $1.04(0.21)$ & $1.24(0.19)$ & $61.7(3.4)$ & $72.6(9.1)$ & $30.8(1.2)^{x}+1$ & $64.2(1.4)$ \\
\hline
\end{tabular}

Table 5.2. Total ( $\mu$ mol.min ${ }^{-1} \cdot \mathrm{g}^{-1}$ wet weight) and tractional activity (\%) of glycogen synthase. * denotes a significant difference between exercised and non-exercised muscies, $\uparrow$ denotes a significant difference between shortening and lengthening exercise.

\section{Muscle GLUT4 content}

Compared to non-exercised controls and shortening exercise, total GLUT4 content declined significantly immediately as well as 24 and $48 \mathrm{~h}$ after exercise. Six $\mathrm{h}$ after lengthening exercise, GLUT4 returned to control values (Fig 5.2, lower panel). Following shortening exercise, GLUT4 content was not significantly affected however, tended to increase 24 and $48 \mathrm{~h}$ post-exercise $(p=0.076$ and $p=0.082$, respectively).

\section{Discussion}

In correspondence with comparable models to study muscle damage following lengthening exercise $(10,23,24,27)$ we observed massive structural muscle damage after lengthening exercise while virtually no damage occurred following shortening exercise.

Muscle glycogen

Immediately post-exercise, muscle glycogen declined to a similar extent following lengthening and shortening exercise. The rate of glycogen restoration during the first $6 \mathrm{~h}$ following shortening exercise $\left(5.4 \mu \mathrm{mol} \cdot \mathrm{g}^{-1} \mathrm{ww} \cdot \mathrm{h}^{-1}\right)$ was significantly lower than following lengthening exercise $\left(3.3 \mu\right.$ mol $\left.\cdot g^{-1} w w \cdot h-1\right)$. As a consequence, glycogen was restored beyond control vallues within $6 \mathrm{~h}$ after shortening exercise, while following lengthening exercise glycogen restored to control values but remained significantly lower than $6 \mathrm{~h}$ after shortening exercise. Different rates of glycogen resynthesis following shortening and lengthening exercise contrast with observations of others reporting comparable rates of glycogen restoration within 6 $h$ following lengthening and shortening exercise (36). In contrast to lengthening exercise, muscle glycogen was maintained at control levels 24 and $48 \mathrm{~h}$ following shortening exercise. 


\section{Enzyme activity}

Despite increased fractional activity of glycogen phosphorylase immediately and 6 $h$ after shortening exercise, glycogen was restored beyond pre-exercise levels within $6 \mathrm{~h}$. Immediately, as well as $6 \mathrm{~h}$ after lengthening exercise, fractional activity of GP was unaffected. Notwithstanding increased fractional activity of GP and possibly glycogenolysis, glycogen was restored to a higher level following shortening than following lengthening exercise during the first $6 \mathrm{~h}$ post-exercise. Increased glycogen content in combination with increased glycogenolysis following shortening exercise, suggests that uptake of glucose following shortening exercise most likely was higher than following lengthening exercise. Declined muscle glycogen $24 \mathrm{~h}$ after lengthening exercise coincided with increased fractional activity of GP together with depressed fractional activity of GS. Enhanced glucose oxidation (partly relying on glycogenolysis) by inflammatory cells following damaging lengthening exercise has previously been hypothesized (7). Increased fractional activity of GP may support this hypothesis. However, inflammation is reported to peak $48 \mathrm{~h}$ after damaging exercise $(1,26,27,35)$. In the present study fractional activity of GP equaled control values at that time. Furthermore, declined muscle glycogen has also been reported following lengthening exercise when inflammation in the exercised muscles was only modest (36) or virtually absent (3). Therefore, the mechanism behind increased fractional activity of GP and subsequent decline in muscle glycogen is most likely not attributable to an inflammatory response in the exercised muscles and remains to be established.

Restoration of muscle glycogen beyond pre-exercise levels within $6 \mathrm{~h}$ after shortening exercise coincided with increased fractional activity of glycogen synthase. Immediately following lengthening exercise, the fractional activity of GS declined significantly and was further depressed for at least $48 \mathrm{~h}$ post-exercise. Literature on the (fractional) activity of GS following lengthening exercise is conflicting. A declined activity of GS has been reported immediately post-exercise upto $72 \mathrm{~h}$ post-exercise (9) while others only observed altered activity of GS immediately post-exercise (7), $24 \mathrm{~h}$ post-exercise (4) or immediately as well as 24 h post exercise (17). Comparison of data from the present study with other studies $(4,7,9)$ is complicated by differences in species, fiber typing of the muscles studied, post-exercise diet, degree of glycogen depletion and the conditions under which the actual enzyme activity was studied (i.e., basal state or high-insulin conditions).

\section{Muscle GLUT4 content}

Following shortening exercise, GLUT4 content was unaltered. In contrast, after lengthening exercise GLUT4 content declined immediately as well as 24 and $48 \mathrm{~h}$ post-exercise. Declined GLUT4 content directly after lengthening exercise is in contrast with reports from Asp and colleagues (4). They showed unaltered muscle GLUT4 content immediately following 40 supramaximal $(25 \mathrm{~V}$ and $100 \mathrm{~Hz}$ ) lengthening contractions. In the present study, muscles were exercised nearly twice as long as in Asp's study. The discrepancy between Asp's study and the present study may originate from differences in exercise intensity and consequently the extent of muscle damage. This notion is substantiated by the inverse 
relationship between muscle damage and GLUT4 content shown by Asp and colleagues (4).

A remarkable and novel observation is that GLUT4 content 6 h after lengthening exercise returned to control values. Increased gene transcription of GLUT4 has been observed as early as $3 \mathrm{~h}$ following a single session of treadmill running (28). Muscle contractions provoked by electrical stimulation as used in the present study may have a similar effect and increased gene transcription of GLUT4 might end up in increased GLUT4 content, as observed in the present study $6 \mathrm{~h}$ after lengthening exercise. Incompatible with this suggestion, however, is the observation that in the present study GLUT4 content is not significantly increased 6 $\mathrm{h}$ after shortening exercise (using the same conditions of electrical stimulation as during lengthening exercise).

Attenuated GLUT4 content 24 and $48 \mathrm{~h}$ following damaging lengthening exercise corresponds with other studies $(3,4)$. During the $48 \mathrm{~h}$ post-exercise period fluctuations in muscle glycogen content are in concert with changes in GLUT4 content, while the fractional activity of glycogen synthase remained depressed for at least $48 \mathrm{~h}$ following lengthening exercise. Glycogen resynthesis is observed within $6 \mathrm{~h}$ after lengthening exercise, despite depressed activity of glycogen synthase. Therefore " we propose that $6 \mathrm{~h}$ after lengthening exercise, muscle GLUT4 content is of more importance for glycogen replenishment than activity of glycogen synthase. This notion is supported by a significant correlation between glycogen replenishment and GLUTT4 content $6 \mathrm{~h}$ after lengthening exercise $\left(R^{2}=0.754, p=0.001\right)$. At the same time, glycogen replenishment and fractional activity of glycogen synthase only showed a weak, non-significant correlation $\left(R^{2}=0.452, p=0.7582\right)$.

Although the mechanism(s) underlying the bi-phasic response of muscle GLUT4 content to damaging lengthening exercise are far from understood, we will propose a hypothetical sequence of events. One of the primary observations following lengthening contraction is disruption of the cellular membrane (6). Upon muscle contraction GLUT4 migrates to, and fuses with, the cellular membrane ( 33 , 34). Due to loss of membrane integrity, GLUT4 may not be able to properlly fuse with the membraine and subsequently be released into the extracellular space. If GLUT4 is no longer a membrane associated protein following lengthening exercise, it can not be ruled out that assessment of GLUT4 content in total crude muscle membranes underestimates the actual GLUT4 content. Contractioninduced glucose transport has a half life time of $-8 \mathrm{~min}$ (31), consequently migration of GLUT4 to the sarcolemma will cease shortly after cessation of exercise, $6 \mathrm{~h}$ post-exercise the majority of GLUT4 will be in intracellular vesicles, thus preventing degradation of the GLUT4 protein. Twenty-four and $48 \mathrm{~h}$ after lengthening exercise myofibrillar protein degradation (25) and cellular remodeling (23) peak and integrity of the GLUT4 containing vesicles may be lost (partly). Subsequently GLUT4 may be exposed to substances degrading the GLUT4 protein.

We conclude that a rapid restoration phase (within $6 \mathrm{~h}$ post exercise) is present following shortening as well as following lengthening exercise. However, the rate of glycogen resynthesis $6 \mathrm{~h}$ following lengthening exercise was 
significantly lower than following shortening exercise. Consequently, muscle gllycogen content $6 \mathrm{~h}$ post-exercise was lower following lengthening exercise compared to shortening exercise. Glycogen resynthesis following llengthening exercise is bi-phasic with restoration to control levels within $6 \mathrm{~h}$, after which glycogen declined again. A novel observation of the present study is that the biphasic response of muscle glycogen following lengthening exercise coincides with a bi-phasic response in muscle GLUT4 content, while fractional activity of glycogen synthase remained depressed the entire post-exercise period.

\section{References}

1. Armstrong, R. B., R. W. Oglivie, and J. A. Scinwane. Eccentric exercise induced injury to skeletal muscle. $J$ Appl Physiol 54: 80-93, 1983.

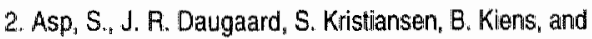
E. A. Richter. Eccentric exercise decreases maximal insulin action in humans: muscle and systemic effects. J Physiol Land 494; 891-898, 1996.

3. Asp, $S_{0}$ J. R. Daugaard, and E. A. Richter. Ecceniric exercise decreases glucose transporter GLUT4 protein in human skeletal muscle. I Physiol Lond 482: 705-712, 1995.

4. Asp, S. S. Kristiansen, and E. A. Richter, Eccentric muscle damage transiently décreases rat skeletal muscle GLUT-4 protein. J' Appl' Physiol 79; 1338-45, 1995.

5. Asp. S., and E. A. Richter. Decreased insulin action on muscle glucose transport after eccentric contractions in rats. J Appl Fhysiol B1: 1924-1928, 1996.

6. Carter, G. T., N. Kikuchi, S. J. Horasek, and S. A. Walsh. The use of fluorescent dextrans as marker of sarcolemmal injury. Histol Histopath 9: 443-447, 1994.

7. Castill, D. L., D. D. Pascoe, W. J. Fink , R. A. Robergs, S. I. Barr, and D. Pearson. Impaired muscle glycogen resynthesis after eccentric exercise. I Appl Physiol 69: $46-50,1990$.

8. Danforth, W. H. Glycagen synthetase activity. Interconversion of two forms and control of glycagen synthesis. J Biol Chem 240: 588-593, 1965.

9. Doyle, J. A., W. M. Sherman, and R. L. Strauss. Effects of eccentric and concentric exercise on muscle glycogen replenishment. \& App/ Physio/ 74: 1848-1855, 1998.

10. Faulkner, J. A., D. A. Jones, and J. M. Round. Injury to skeletal muscies of mice by forced lengthening during contractions. Q J Exp Physiol 74: 661-670, 1989.
11. Ferry, A., 1. Amiridis, and M. Rieu. Glycogen repletion and synthesis in the rat after downhill running. Eur J Appl Physiol 64: 32.35, 1992.

12. Fitts, R.H. Cellular mechanisms of muscle tatigue. Physiological reviews 74: 49-94, 1994.

13. Furler, S. M., A, B. Jenkins, L. H. Storliem, and E. W. Kraegen. In vivo location of the rate limiting step of hexose uptake in muscle and brain tissue of rats. Am J Physiol261: E337-E347, 1991.

14. Goodyear, L. J., M. F. Hirshman, P. M. Walyou, and E. S. Horton, Glucose transporter number, function, and subcellular distribution in rat skeletal muscle after exercise training. Diabetes 41: 1091-9, 1992.

15. Harris, R. C., E. Hultman, and L.-O. Nordjesjö. Glycogen, glycolytic intermediates and thigh-energy phosphates determined in biopsy samples of musculus quadriceps femoris of man at rest. Methods and variance of values. Scan J Clin Lab invest 33: 109$120,1974$.

16. Hesselink, M. K. C., M. R. Drost, J. Komulainen, R. Uolita, H. A. Keizer, and H. Kuipers. Increased contractile wark during tengthening contractions after shortening and lengthening training. Submilted" 1997.

17. Hesselink, M. K. C., H. Kuipers, H. A. Keizer, M. R. Drost, and $G$. J. van der Vusse. Acule and sustained eifects of isometric and lengthening muscle contractions on high-energy phosphales and glycogen metabolism in rat tibialis anterior muscle. $d$ Muscle Research and Cell Motility Accepted, 1997.

18. Ivy U. L. Muscle glycogen synthesis berore and after exercise Sports Med 11: 6-19, 1991.

19. Iwy, J. L. A. L. Kalz, C. L. Culler, W. M. Sherman, and E. F. Coyle. Muscle glycogen synthesis after exercise: effect of time of carbohydrate ingestion. J Appl Physiol 64: 1480-5, 1988. 
20. IWy, J. L., M. C. Lee, J. J. Brozinick, and M. J. Peed. Muscle ghcogen storage after different amounts of carbohydrate ingestion. IAppl Physiol 65: 2018-23, 1980:

21. Keizer, H. A. H. Kuipers, Gi, van Kranenbuifg, and $P$. Geurten. Intivence of liquid and solid meals on muscle glycogen resynthesis, plasma fuel homone response. and maximal physical working capacity. Int J Sports Med 8: 99-104, 1987.

22. Kristiansen, S., S. Asp, and E. A. Richter. Decreased muscle glut 4 and contraction induced glucose iransport after eccentric contractions. American Journal Of Physiology Regulatory Integrative And Comparative Physiology. Aug 40: R477-R482, 1996.

23. Lieber ${ }_{i}$ R. L., M. C. Schmitz, D. K. Mishra, and d. Friden. Contractile and cellular remodeling in rabbit skeletal muscle after cyclic eccentric contractions. I Appl Physiol 77: 1926-34, 1994.

24. Liaber, A. L. T. M. Woodburn, and J. Friden. Muscle damage induced by eccentric contractions of 25\% strain. J Appl Physiol 70: 2488-507, 1991.

25. Lowe, D. A., G. L. Warren, C. P. Ingalls, D. B. Boorstein, and R. B. Armstrong. Muscle function and protein metabolism after initiation of eccentric contraction-induced injury. J Appi Physiol 79: 1260-1270, 1995.

26. Macintyre, D. L., W. Darlene Reid, and D. C. McKenzie. Delayed muscle soreness, The inflammatory response to muscle injury and its clinical implications. Sports Med 20: 24-40, 1995.

27. MoCully, K, K., and J. A. Faulkner. Injury to skeletal muscle libers of mice lollowing lengthening contractions. J Appl Physiol 59: 119-126, 1985.
26. Neufer, P. D., and G. L. Dohm Exercise induces; a transient increase in transcription of the GLUT-4 gene in skeletal muscle. Am s Physiof, 1993.

29. OReilly, K. P. M. J. Warhol, R. A. Fielding, W. R. Frontera ${ }_{n}$ C. N. Meredith, and W. J. Evars. Eccentric exercise-induced muscle damage impairs muscle glycogen repletion. J Apol Physiol 63: 252-256, 1987.

30. Pascoe, D. D., D. L. Costill, W. J. Fink, R. A. Robergs, and $\mathrm{J}$. J. Zachwieja. Glycogen resynthesis in skeletal muscle following resistive exercise. Med Sci Sports Exerc 25: 349-354, 1993.

31. Ploug, T., H. Galbo, J Vinten, $M$. Jargensen, and $E$. A. Richter. Kinetics of glucose transport in rat muscle: effects of insulin and contractions. Am J Physiol Endocrinol Metab 16: E12-E20, 1987.

32. Ren, J.-M. B. A. Marshall, E. A. Gulve, J. Gao, D.W. Johnson, J. O. Holloszy, and M. Mueckler. Evidence from transgenic mice that glucose transport is rate limiting for glycogen deposition and glycolysis in skeletal muscle. J Biol Chem 268: 16113-16115, 1993.

33. Rodnick, K. J., R. C. Piper, J. W. Slot, and D. E. James, Interaction of insulin and exercise on glucose transport in muscle. Diaboles care 15: 1679-1689, 1992.

34. Rodnick, K. J., J.W. Slot, D. R. Studelska, D. E. Hanpeter "L. J. Robinson, H. J. Geuze, and D. E. James. Immunocytochemical and biochemical studies of GLUT4 in rat skeletal muscle. The jounar of biological chemistry 267: 6278-6285, 1992.

35. Smith, L. L. Acute inflammation: the underlying mechanism in delayed onset muscle soreness? Med Sci Sports Exerc23: 542-551, 1991.

36. Widrick, J. J. D. L Costill, G. Ku McConel, D. E. Anderson, D. R. Pearson and J. J. Zachwieja. Time course of glycogen accumulation after eccentric exercise. J Appl Physial 72: 1999-2004, 1992. 


\section{6}

\section{Immunolocalization of GLUT4 at Light Microscopical Level in Rat Cardiac and White Gastrocnemius Muscle}

M.K.C. Hesselink, L.B. Borghouts, H. Kuipers, H.A. Keizer

Submitted. 


\begin{abstract}
Transmembrane glucose transport in muscle relies largely on the presence of the glucose transporting protein GLUT4. The present study was conducted to qualitatively examine the localization of GLUT4 at light microscopical (LM) level in cardiac and white gastrocnemius muscle by immunofluorescence under resting conditions and after insulin administration, or contractile activity. Indirect immunofluorescence using an affinity purified antibody against the 15-C terminus of GLUT4 was employed on sections of cardiac muscle and white gastrocnemius, both upon insulin administration $\left(8 \mathrm{U} \cdot \mathrm{kg}^{-1} \mathrm{bw}\right.$, i.p.) or muscle contraction induced by electrical stimulation ( 240 contractions, $300 \mathrm{~ms}$ pulse trains, $150 \mathrm{~Hz}, 3 \mathrm{~V}$ ). In cardiac muscle, label was more abundant than in resting white gastrocnemius muscle and was also present intracellularly. In resting white gastrocnemius only weak labeling was observed in vicinity of the cell border. Upon insulin administration or contraction labeling appeared to be intensified at the cell borders. We conclude that labeling of GLUTA by immunofluorescence is useful to localize GLUT4 at LM in red, oxidative cardiac muscles and to a lesser extent in white glycolytic muscles. Translocation of GLUT4 in rat white gastrocnemius muscle after insulin administration and exercise can be qualitatively observed by immunofluorescence at LM level.
\end{abstract}




\section{Introduction}

A large portion of post-prandial glucose is taken up by skeletal muscle. Transmembrane transport of glucose is the rate limiting step for glucose utilization $(3,19)$ and increases several fold upon stimulation with insulin as well as during and following muscle contraction $(4,7,15)$.

Channeling glucose from the extracellular space into the cytosol relies largely on diffusion facilitated by specific glucose transporters of which GLUT1 and GLUT4 have been identified to be the main glucose transporting proteins in skeletal muscle (14). Subcellular fractionation studies have shown that GLUT1 is found almost exclusively in the plasma membrane (9) while GLUT4 is recruited to the plasma membrane upon stimulation with insulin or after muscle contractile activity $(12,13)$. Under resting conditions the majority of GLUT4 is localized in intracellular vesilcles close to the plasma membrane (20). Upon stimulation with insullin or following muscle contraction, GLUT4 content of microsomal membranes declines, with a concomitant increase in plasma membranes $(5,8,21)$.

Translocation of GLUT4 is mostly studied by subcellular fractionation $(5,8$, 21). Other techniques applied to study the translocation process are exofacial photolabeling $(2,16)$ and immunocytochemistry at electron microscopical level (1, $20,22,23,25)$. However, none of these techniques can be easilly combined with fiber typing and/or PAS-staining for glycogen content.

Prolonged endurance training induces alterations in GLUT4 and muscle glycogen content, as well as a shift towards more oxidative muscle fibers. The proportion of oxidative fibers correlates highly with the muscle GLUT4 content ( $R=$ $0.94(18)$ ). Immunohistochemical stains of serial muscle tissue sections at light microscopical (LM) level makes it possible to study distribution and localization of GLUT4 in different fiber types.

In the present study we examined whether the localization of GLUT4 could be studied qualitatively by immunofluorescence in rat cardiac muscle (predominantly consisting of highly oxidative fibers) and the white part of rat gastrocnemius muscle (a predominantly glycolytic muscle). These types of tissue were chosen to contrast between a tissue expressing GLUT4 abundantly (cardiac muscle) and a tissue with a low GLUT4 expression. Furthermore, it was studied whether differences in the localization of GLUT4 in rat white gastrocnemius were detectable at light microscopical level upon insulin administration or muscle contraction.

\section{Materials and methods}

\section{Animals}

In the present study 12 malle mature Wistar rats (Charles River) were used. Exercise, insulin administration and muscle dissection were performed under pentobarbital anesthesia (Narcovet ${ }^{(\mathcal{B}} 1.0 \mathrm{ml} \cdot \mathrm{kg}^{-1}$ bady weight, i.p. injection) after short-term (less than 20 s) $\mathrm{CO}_{2}$ sedation. After muscle dissection, rats were sacrificed by cervical dislocation. All experimental procedures were approved by the Institutional Animal Care and Use Committee of the Maastricht University and complied with the principles of laboratory animal care. 


\section{Experimental procedures}

The rats were randomly assigned to 1 out of 3 experimental conditions. One group $(n=4)$ was injected intraperitoneally (i.p.) with $1 \mathrm{ml}$ saline, serving as a control group. A second group $(n=4)$ was injected i.p. with $1 \mathrm{ml}$ saline containing 8 units bovine insulin (Actrapid, Novo Nordisk) per kg body mass. In the third group ( $n=4$ ), the triceps surae muscle complex was exercised by electrical stimulation at the exposed ischiadic nerve. During exercise rats performed 4 sessions of 60 supramaximal shortening contractions, using $300 \mathrm{~ms}$ pulse trains (150 Hz, 3V) every 3 seconds. Successive exercise sessions were interspaced by a 3 minute recovery period. All rats had free access to food and tap water until $1 \mathrm{~h}$ before the start of the experiment. Within $1 \mathrm{~h}$ before the start of the experiment rats were deprived from food. Anesthesia was maintained until sacrifice.

Thirty minutes after administration of either insulin or saline as well as 30 min after termination of the exercise protocol, the white part of the gastrocnemius muscle was excised. From rats injected with saline, the heart was excised after thoracotomy. From all rats the midbelly region of the white gastrocnemius and the apex of the heart were mounted on a thin piece of cork with O.C.T. embedding compound (Tissue-tek ${ }^{\circledR}$, Miles Laboratories). Subsequently, samples were frozen in isopentane cooled to melting point with liquid nitrogen and stored at $-80^{\circ} \mathrm{C}$ until analyses.

\section{Immunofluorescence labeling}

For detection of GLUT4, frozen muscle samples were cryosectioned and the obtained sections were processed for immunolabeling. Unfixed, serial transverse cryosections of $5 \mu \mathrm{m}$ were cut at $-20^{\circ} \mathrm{C}$ and thaw-mounted on uncoated glass slides. Promptly after air drying for $\sim 20 \mathrm{~min}$, labeling started with a 3 times wash (2 $\mathrm{min}$ ) in $0.1 \mathrm{M}$ phosphate buffered saline (PBS) followed by a 30 min blocking step with $10 \%(\mathrm{~V} / \mathrm{v})$ newborn calf serum (NCS), $5 \%$ bovine serum allbumin (BSA) (w/v) and $0.1 \%(\mathrm{w} / \mathrm{v})$ coldwater fish skin gelatin (CWFSG) in $0.1 \mathrm{M}$ PBS. Upon blocking, the sections were rinsed ( 3 times 2 minutes) in the same medium ( $0.3 \%$ BSA $(w / v)$ in $0.1 \mathrm{M} \mathrm{PBS}$ ) used for dilution of the antibodies. Thereafter, the sections were incubated for $45 \mathrm{~min}$ with an affinity purified polyclonal antibody raised in rabbits against the 15-C terminus of rat GLUT4 (kindly provided by Dr. A. Zorzano, Barcelona, Spain) diluted $1: 50(\mathrm{v} / \mathrm{v})$ in $0.1 \mathrm{M}$ PBS containing $0.3 \%$ BSA (w/v). Incubation with the primary antibody was followed by a 3 times 2 min rinse in $0.1 \mathrm{M}$ PBS containing $0.3 \% \mathrm{BSA}(\mathrm{w} / \mathrm{v}$ ) after which the sections were incubated for $45 \mathrm{~min}$ with the secondary antibody (fluorescein isothiocyanate (FITC)-conjugated swineanti-rabbit IgG, Dakopatts, Copenhagen, Denmark). This incubation step was followed by a 3 times 2 min rinse in $0.1 \mathrm{M}$ PBS containing $0.3 \% \mathrm{BSA}(\mathrm{w} / \mathrm{v})$. Subsequently, the sections were rinsed in $0.1 \mathrm{M}$ PBS (2 times $5 \mathrm{~min}$ ) and were finally mounted in 1,4-diazobicyclo-[2,2,2]-octane (DABCO) with 4,6-diamidino-2phenylidole (DAP!) (Sigma, St. Louis, USA).

Two controls were included, a negative control in which the primary antibody was replaced by $0.1 \mathrm{M}$ PBS containing $0.3 \% \mathrm{BSA}(\mathrm{w} / \mathrm{v})$, and a positive control when a polyclonal antibody raised in rabbits against myosin $S 1$ fragments (Sigma, St. Louis, USA) was used as the primary antibody (diluted 1:10 in $0.1 \mathrm{M}$ PBS 
containing $0.3 \% \mathrm{BSA}(\mathrm{w} / \mathrm{w}))$. The entire labeling procedure was performed in a moisture chamber at room temperature.

All sections were examined and photographed using a Zeiss Axioskop (Carl Zeiss, Oberkochen, Germany) within $24 \mathrm{~h}$ after the labeling procedure.

\section{Results}

In sections where the primary antibody was omitted, no label was observed (picture not shown). Using the polyclonal antibody against the S1 fragment of rat myosin as a primary antibody, label was abundantly present in the sarcoplasm and absent in the sarcolemma and in nucleil (picture not shown).

In cardiac muscle, GLUT4 labeling was present in the cell interior as well as at the cell periphery and was clearly absent in nuclei (Fig 6.1, upper left panel). Under basal conditions the presence of GLUT4 in white gastrocnemius muscle was far less abundant than in cardiac muscie and weak labeling was observed aimost exclusively in the vicinity of cellular membranes (Fig 6.1 upper right panel). Upon stimulation with insulin (Fig 6.1 lower left panel) or following contractile activity (Fig 6.1 lower right panell) the signal in the proximity of cellular membranes appears to be intensified compared to the basal state. Intensification of label was most pronounced close to the nuclei.
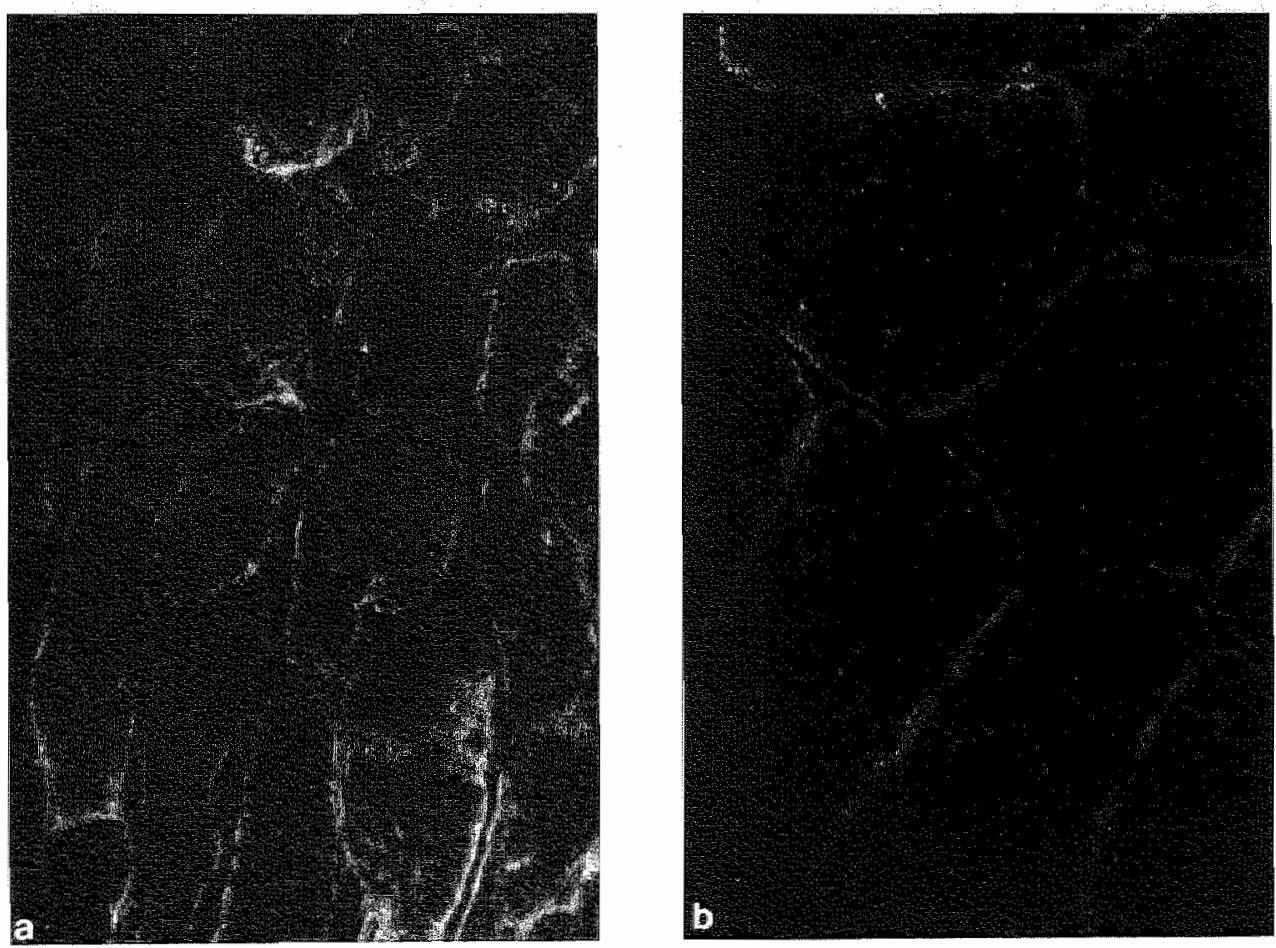

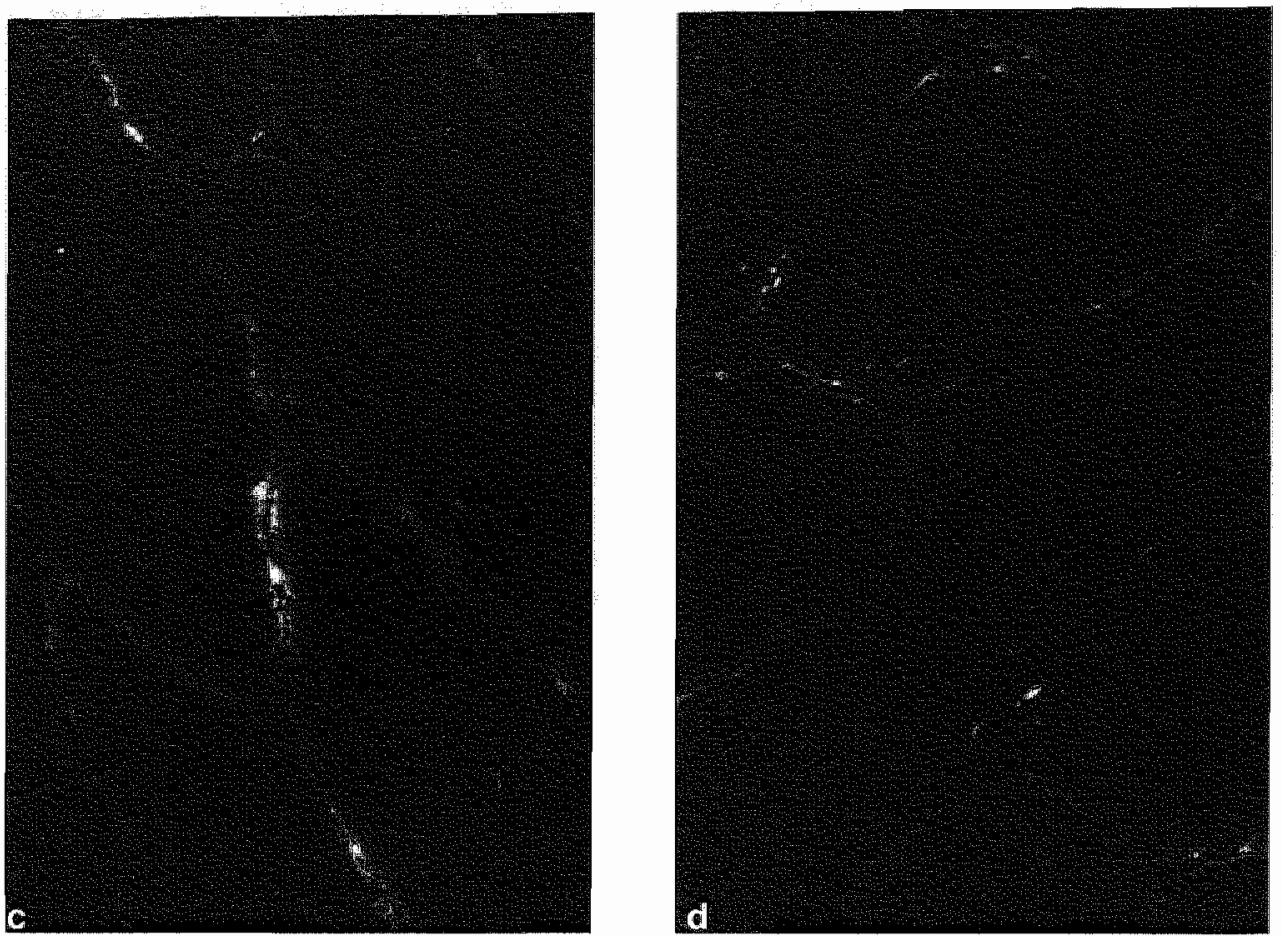

Figure 6.1: Panel a shows immunolocalization of GLUT4 in unstimulated cardiac muscle (note the presence of label in both the cell interior as well at the cell periphery). Panel b shows immunolocalization of GLUT4 in white gastrocnemius muscle under basal conditions (note that label is far less abundant than in cardiac muscle and is restricted to the vicinity of the cell border). Panels $c$ and $d$ respectively depict immunofluorescence in white gastrocnemius muscle after insulin stimulation or after contraclile activity. Compared to the basal state label in the proximity of cellular membranes appears to be intensified at sites close to the nuclei. Photomicrographs shown are representative pictures fram the muscles examined. All pictures were taken using identical exposure times.

\section{Discussion}

The present study was conducted to qualitatively examine the localization of GLUT4 at light microscopical (LM) level in cardiac and white gastrocnemius muscle by immunofluorescence under resting conditions and after insulin administration, or contractile activity.

The almost complete absence of label in the control sections where the primary antibody was omitted implicates a low cross reactivity of the secondary FITC-conjugated swine-anti-rabbit antibody used. The abundant labeling observed using the rabbit anti-myosin $S 1$ serum implicates proper recognition of rabbit IgG by the secondary FITC-conjugated swine-anti-rabbit antibody. Therefore, the signal detected in sections incubated with the affinity purified GLUT4 antibody represents specific labeling of GLUT4.

Previous reports on localization of GLUT4 in cardiac tissue at electron microscopical (EM) level revealed that under unstimulated conditions, GLUT4 was associated with tubullo-vesicular elements near the sarcolemma and to a lesser 
extent) near the intercalated discs (23). Labeling was observed at the transverse tubular system in the Z-line area and randomly throughout the sarcoplasm as well (23). Although the above mentioned specific structures can not always be identified at $L M$ level, distribution of the tubulo-vesicular elements and intercalated discs at LM level is such that labeling of GLUT4 in vicinity of the cellular membrane is to be expected. Labeling of the transverse-tubular system in the Z-line area together with sarcoplasmic labeling may account for the presence of label in the cell interior. Therefore, observed labeling of both the cell periphery and the cell interior in unstimulated cardiac muscle in the present study corresponds with previous findings at EM level (23) as well as with the only report found on GLUT4 labeling at LM level using immunofluorescence (26).

In the white (predominantly glycolytic) gastrocnemius muscle, GLUT4 labeling under basal conditions is far less abundant than in highly oxidative red cardiac muscle. This observation is in accordance with reports showing that GLUT4 expression is higher in oxidative than in glycolytic muscle $(10,13,18)$. However, the abundance as well as the site of GLUT4 label in basal white gastrocnemius is in sharp contrast with results of Marette et al. (17), who showed abundant labeling of GLUT4 in both the cell interior as well as at the cell periphery. The discrepancy between the present study and the study of Marette et al. (17) most likely originates from differences in the duration of incubation with the primary antibody. In the present study we have used an aftinity purified polyclonal antibody raised in rabbits against the $15-\mathrm{C}$ terminus of rat GLUT4. Instead of overnight incubation at $4^{\circ} \mathrm{C}$ with the monocional $1 F 8$ antibody (17), we exposed the sections 45 minutes to a polyclonal GLUT4 antibody at room temperature. When doing so we only observed a weak labeling in the vicinity of the nuclei close to cell border, while virtually no label was detected in the sarcoplasm. These observations are in line with reports showing that labeling of GLUT4 after short-term incubation ( $30-60 \mathrm{~min}$ ) with the 1F8 antibody was confined to regions of the cell borders of the myocytes of rat soleus muscle without clear labeling of the Z-lines (24).

Furthermore, we have shown that immunogold silver staining (IGSS) or AEC-peroxidase staining using a commercially available monoclonal antibody against partially pure rat GLUT4 (21/71 Biogenesis, UK) as a primary antibody yielded comparable results as shown in the present study (11) (data from IGSS are shown in Fig 6.2).

Both upon stimulation with insulin as well as after muscle contraction, labeling appaers to be intensified in proximity of the cell border, which is agreement with observations using subcellular fractionation reporting increased GLUT4 content in plasma membrane fraction after exercise or stimulation with insulin (6-8). Reports on immunolocalization of GLUT4 at LM level after insulin administration or contractile activity are scarce. In rat soleus muscle it is reported that changes in the labeling pattern of GLUT4 after insulin stimulation are not as obvious as in brown adipose tissue (24), unfortunately the authors did not show a photomicrograph of GLUT4 labeling in skeletal muscle following insulin administration. 

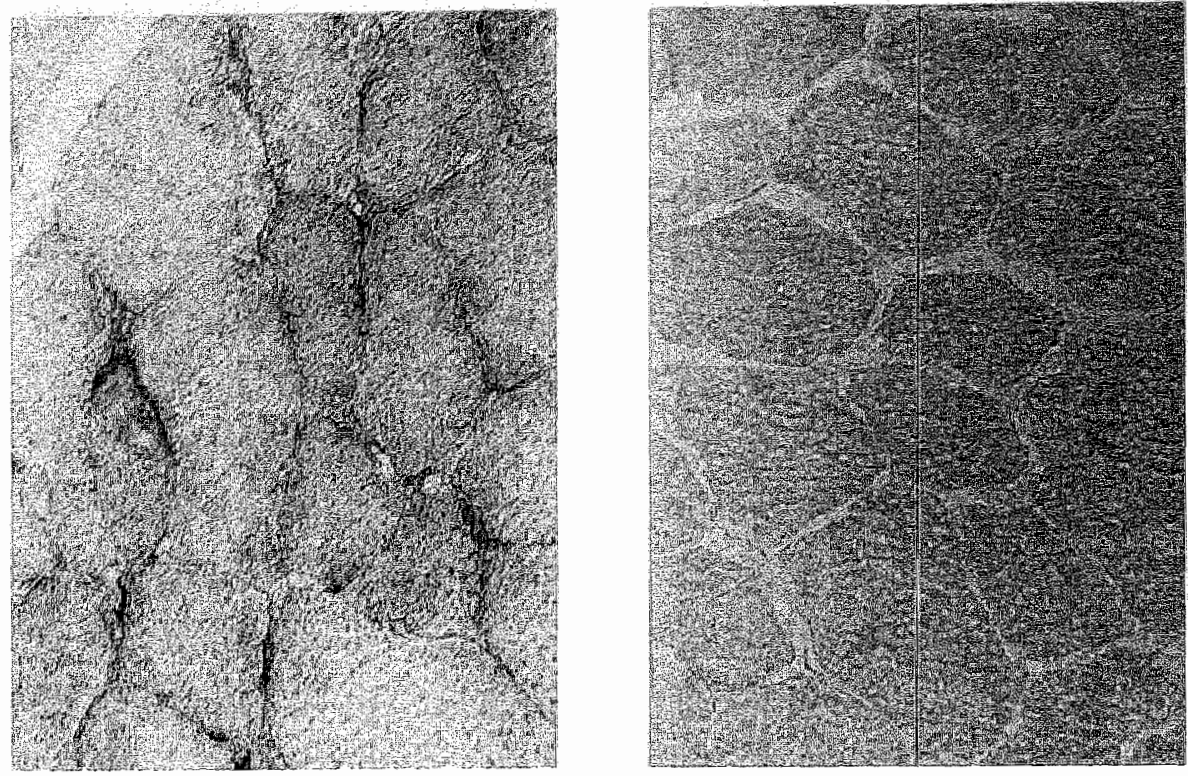

Figure 6.2: Detection of GLUT4 by immunogold silver staining in basal white gastrocnemius muscle after incubation with a monoclonal antibody (21/71. Biogenesis) against GLUT4 (left panel). GLUT4 was localized by a secondary nano-gold conjugated antibody. Subsequently, nano-gold complexes were visualized at $L M$ level by silver enthancement, bringing about a dark stain. Following this procedure label was predominantly present in the vicinity of the cell border while only little labell was detected in the cell interior. In control sections the anti-GLUT4 was replaced by PBS containing $1 \%$ (w/w) BSA (right panel), note the almost complete absence of label in the control sections.

In conclusion, the present study shows that immunofluorescence labeling of GLUT4 is a promising technique to localize GLUT4 at light microscopical level in muscles with a relatively high expression of GLUT4 (red muscles, in the present study cardiac muscle) and to a lesser extent to localize GLUT4 in white glycolytic muscles. To the best of our knowledge, the present study is the first to indicate that immunofluorescence at LM level can be used to qualitativelly study translocation of GLUT4 in rat skeletal muscle upon stimulation with insulin or exercise. More work is required to permit quantification of the signal, and thus permit differentiation between the extent of insulin- or contraction-mediated translocation of GLUT4. Since differentiation between muscles expressing GLUT4 abundantly and muscles with a low GLUT4 expression is permitted by immunofluorescence, immunolabeling of GLUT4 will be helpful to study training induced changes in muscle GLUT4 content.

\section{Acknowledgment}

The authors are greatly indebted to A. Zorzano from the University of Barcelona for the generous gift of the antibody. The assistance of Rein van Gool during the immunostaining procedures is gratefully acknowledged. 


\section{References}

1. Bomemann, A., T. Ploug, and H. Schmalbruch. Subcellular localization of GLUT4 in nonstimulated and insulin-stimulated rat soleus muscle. Diabetes 38: 215-221, 1992.

2. Dudek, R. W., G. L. Dohm, G. D. Holman, S. W. Cushman, and C. M. Wilson. Glucose transporter localization in rat skeletal muscle. Auto-radiographic stduy using ATB-(2-3H)BMPA photolabel. FEBS Lett 39: 205-208, 1994.

3. Furler, S. M., A. B. Jenkins, L. H. Storlien, and E. W. Kraegen. In vivo location of the rate limiting step of hexose uptake in muscle and brain tissue of rats. Am $J$ Physio/261: E337-E347, 1991.

4. Goldstein, M. S. S. V. Mullick, B. Huddlesiun and R. Levine. Action of muscular work on transter of sugars across cell barriers: comparison with action of insulim. Am J Physiol 173: 212-216, 1953.

5. Goodyear, L. J., M. F. Hirshman, and E. S. Horton. Exercise-induced translocation of skeletal muscle glucose transporters. Am J Physiol 0054, 1991.

6. Goodyear, L. J., M. F. Hirshman, R. Napoli, J. Calles, J. F. Markuns, O. Ljungqvist, and E. S. Horton. Glucose ingestion causes GLUT4 trans ocation in human skeletal muscle. Diabetes $45_{*}^{*} 1051-6,1996$.

7. Goodyear, L. J., M. F. Hirshman, R. J. Smith, and E. $S$. Horton. Glucose transporter number, activity, and isoform content in plasma membranes of red and white skeletal muscie. Am J Physiol 0054 1991.

8. Goodyear, L. J., P. A. King, M. F. Hirshman, C. M. Thompson, E. D. Horton, and E. S. Horlon. Contractile actiwity increases plasma membrane glucose transporters in absence of insulin. Am J Physiol 0054, 1990.

9. Gould, G. W., and G. D. Holman. The glucose transporter tamily: structure, function and lissuespecific expression. Biochem J295: 329-341, 1993.

10. Herriksen, E. J., F. E. Boumey, K. J. Fodnick, L. Koranyi, M. A. Permutti, and J. O. Holloszy. Glicose Iransporter protein content and glucose transport capacily in ral skeletal muscles. Am J Physiol 259: 593 598, 1990.

11. Hesselink, M. K. C., E. Breda van, P. Geurten, H. Kuipers, and $H$. A. Keizer. Immunolocalization of GLUT 4 in rat gastrocnemius muscle by light microscopy. Eur J Physiol 430: R69, 1995.
12. James, D. E. R. Brown, J. Navario, and P. F. Pilch. Insulin-regulatable tissues express a unique insulin. sensitive glucose transpont protein. Nature 333: 183 $185,1988$.

13. James, D. E., M. Strube, and M. Mueckler. Molecular cloning and characterization of an insulinregulatable glucose transporter. Nature 338: $83-87$. 1989.

14. Klip. A., and M. R. Pâquet. Glucose transport and givcose transporters and their metabolic regulation. Diabeles care 13: 228-243, 1990.

15. Levine, R., M. S. Goldstein, B. Huddlestum, and S. P. Klein. Action of insulin on the "permeability" of cells to tree hexoses "as studied by its effecl on the distribution of galactose. Am J Physiol 163: 70-76, 1950.

16. Lund, S., G. D. Holman, O. Schmitz, and O. Pedersen. GLUT4 content in the plasma mernbrane of rat skelelal muscle: comparative studies of the subcellular fractionation method and the exofacial photolabelling lechnique using ATB-BMPA. FEBS Lett 312-318; 1993.

17. Marette, A., J. M. Richardson, T. Aamlal, T. W. Ballon, M. Vranic, J. E. Pessin, and A. Klip. Abundance, localization, and insulin-induced translocation of glucose transporters in red and white muscle. Am J Physiol, 1992.

19. Miegeney, L. A., P. D. Neufer, G. L. Dohm, M. H. Tan, C. A. Blewett, G. C. Elder, and A. Bonen. Effects of muscle activity and fiber composition on glucose transport and GLUT-4. Am J Physiol, 1993.

19. Fen, J.M., B. A. Marshall, E. A. Guive, J. Gao, D. W. Johnson, J. O. Holloszy, and M. Mueckler. Evidence from transgenic mice that glucose fransport is rate limiting for glycogen deposition and glycolysis in sketetal muscle. . Biol Chem 268: 16113-16115, 1993.

20. Rounick, K. J., J.W. Slot, D. R. Studelska, D. E. Hanpeter, L. J. Robinson, H. J, Geuze, and D. E. James. Immunocytochemical and biochemical sludies of GLUT 4 in rat skeletal muscle. The jounal of biological chemistry 267: 6278-6285, 1992.

21. Sherman, L. A., M. F. Hirshman, M. Cormont, M. B. $Y$. Le, and L. J. Goodyear. Differential effects of insulin and exercise on Rab4 distribution in rat skeletal muscle. Endocrinology 137: 266-73, 1996. 
22. Slot, J.W., H. Geuze, S. Gigengack, G. E. Lienhard, and $D$. E. James: Immuno-localization of the insulin regulatable glucose transporter in brown adipose lissue of the rati. $J$ Cell Biol 113: $123-135,1991$.

23. Slol, J.W. H. J. Geuze, S. Gigengack, D. E. James, and $G$. E. Lienhard. Transiocation of the glucose Iransporter GLUT4 in cardiac myocytes of the rat. Proc Natf Acad Sc USA 8B: 7615-7819, 1991.

24. Stot, J. W. R. Moxley, H. J. Geuze, and D. E. James. No evidence for expression of the insulin-regulatable glucose lransporter in endothelial cells. Nafure 346 : $369-371_{1} 1990$.
25. Smith, R. M., M. J. Charron, N. Shah, H. F. Lodish, and L. Jarett. Immunoelectron microscopic demonstration of insulin-stimulated translocation of glucose transporters to the plasma membrane of isolated ral adipocytes and masking of the carboxy. terminal epittope of intracellular GLUT4. Proc Natl Acad SciU SA 88: 6893-7, 1991.

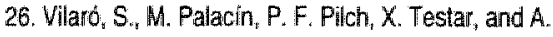
Zorzano. Expression of an insulin-regulatable glucose carrier in muscle and fal endothelial cells. Nature 342 : $798-800,1989$. 


\section{Increased Contractile Work During Lengthening Contractions After Shortening and Lengthening Training}

M.K.C. Hesselink, M.R. Drost, J. Komulainen 1, R. Uotila ", H.A. Keizer, H. Kuipers ${ }^{1}$ LIKES-Research Center, University Campus, Jyväskylä, Finland.

Submitted. 


\section{Abstract}

In the present study we aimed to investigate the repeated bout effect with emphasis on indices of muscle damage, on contractile work during successive lengthening contractions and on the total amount of work performed during successive exercise bouts. Rat hindlimb dorsiflexor muscles were subjected to either shortening or lengthening exercise by electrical stimulation. Fifteen days later, muscles subjected to prior shortening or prior lengthening exercise both performed lengthening exercise. Muscle function was evaluated using a rat dynamometer which enables assessment of the amount of work performed during muscle contraction. Muscle morphology and B-glucuronidase (B-GU) activity were studied for evaluation of muscle damage. Muscle morphology was found to be preserved and B-GU activity was blunted in muscles performing lengthening exercise for the second time. Preserved muscle morphology was functionally reflected in a less severe decline in contractile work and an increased amount of total work performed (i.e., decreased fatiguability) during a second lengthening exercise session. These adaptive responses were absent in muscles previously subjected to shortening exercise. The present study shows that a 'repeated bout effect' is present exclusively if the initial exercise session comprises lengthening exercise, induces preserved muscle morphology and an improvement in muscle contractile work combined with decreased fatiguability. 


\section{Introduction}

Eccentric exercise or lengthening contractions induces transient muscle damage (for review see $1,2,8,30$ ). When a second session of lengthening contractions is employed within $1-10$ weeks after the first exercise session, the deleterious effects of lengthening exercise on indicators of muscle damage are less severe $(6,20,22)$. This rapid adaptation to lengthening exercise, known as the 'repeated bout effect', is reported aiready after one session of lengthening contractions $(4,6,7,17,20$, $21,25,28,29$ ).

In the majority of human studies investigating the repeated bout effect, the extent of muscle damage was assessed indirectly and muscle function was tested by recording maximal isometric force after a lengthening exercise session. A decline in the amount of work performed during successive shortening contractions (SC) was reported only in association with metabolic changes, while during lengthening contractions (LC) work declined progressively even in the absence of metabolic changes (31). A decline in the amount of work performed during lowintensity successive $L C$ is associated with muscle damage (31). During successive high-intensity LC, muscle damage is also accompanied by metabolic changes. Therefore, it is conceivable that contractile work during high-intensity exercise declines more during lengthening than during shortening exercise. A previously employed session of LC may alleviate the extent of muscle damage induced by a second session LC (repeated bout effect) and contractile work will decline less than during the first lengthening exercise session (31).

Most of the reports on the repeated bout effect only recorded maximal isometric force after lengthening exercise. From a functional perspective the amount of work performed during a contraction more closely reflects normal muscle functional capacity than isometric force. To date, information on the amount of work performed by muscles during repeated lengthening exercise is lacking.

In the present study we examined the effect of prior lengthening exercise on the amount of contractile work performed during a second lengthening exercise session, together with the extent of muscle damage induced by the second session of LC. We hypothesize that muscle damage is less prominent following a second lengthening exercise session (if performed within $15 \mathrm{~d}$ after the first lengthening exercise session). Furthermore, we hypothesize that the amount of work performed during a second lengthening exercise session will decline less than during the first lengthening exercise session. Finally, the relative decline in work is hypothesized to be less during a second lengthening exercise session. Consequently, the total amount of work performed during a second lenghthening exercise session is hypothesized to be increased.

Work during lengthening exercise was recorded in rats in vivo using a custom-built, previously unpublished dynamometer. Maximal contractile activity was achieved by electrical nerve stimulation. Muscle damage was assessed directly by studying muscle morphology as well as indirectly by activity of Bglucuronidase. The indirect marker used to assess the extent of muscle damage, Bglucuronidase, is known to correlate highly $\left(\mathrm{A}^{2}=0.873\right)$ with the histo-pathological state of the exercised muscle (27). 


\section{Materials and methods}

\section{Dynamometer characterization}

The experimental set-up consists of 4 main units (Figure 2.1), a custom built Ashton-Miller like (3) rat dynamometer (RDM), a motion control unit to adjust RDM settings (e.g., rotational velocity and range of motion), an Apple Macintosh 7100 PowerPC ${ }^{B}$ with an 8 channel, 12 bits Lab-NB analog-to-digital conversion board (National Instruments) and a pulse generator for electrical stimulation and triggering of the RDM (HSE 215/IZ, Freiburg, Germany).

The RDM consists of a platform with 6 degrees of freedom, a femur fixation unit, a rotational footplate, a linear motor and a potentiometer. An anaesthetized rat is placed in supine position on the heated $\left(37^{\circ} \mathrm{C}\right)$ platform. Fixation of the femur prevents lateral movement of the knee and is achieved by two conical pieces, placed on the skin between the femoral and tibial condyles. To prevent transmission of force through the tibia to the femur fixation unit, the femur fixation unit is connected to the body of the RDM by a hinge construction. The ankle rotation axis is aligned with the rotation axis of the RDM by translation of the footplate relative to the rotation axis of the RDM in 2 perpendicular directions.

Rotation is induced by a linear motor. Total torque is determined from the voltage through the linear motor of the RDM and equals the sum of frictional and inertial torque of the mobile system, muscle passive torque and, if any, muscle active torque. Muscle active torque was obtained by subtracting torque during a passive stroke (i.e., to a movement without muscle contraction) from the torque during muscle contraction (active stroke). External work of the active muscle group is computed as the integration of active torque $(\mathrm{Nm})$ over rotation angle (rad) curve.

To monitor muscle passive torque during exercise, all exercise bouts started and ended with recording muscle passive torque. To obtain active muscle work, torque of the passive strokes was subtracted from all subsequent active strokes. To ensure adequate muscle tension before rotation, a $210 \mathrm{~ms}$ delay was maintained between the onset of nerve stimulation and initiation of rotation in the ankle joint.

\section{Animals}

The present study was conducted on tibialis anterior (TA) and extensor digitorum longus (EDL) muscles of the hindlimbs of male Wistar rats (Charles River), aged 12 weeks at the onset of the study. Exercise and muscle dissection were performed under pentobarbital anesthesia (Narcovet ${ }^{\circledR} 1.0 \mathrm{ml} \cdot \mathrm{kg}^{-1}$ body weight, i.p. injection) after short-term (less than $20 \mathrm{~s}$ ) $\mathrm{CO}_{2}$ sedation. After muscle dissection, rats were sacrificed by cervical dislocation. All experimental procedures were approved by the Institutional Animal Care and Use Committee of the Maastricht University and complied with the principles of laboratory animal care.

Percutaneous needle electrode positioning

For electrode positioning, the skin was depilated just distal of the caput fibulae at the lateral side of the hindlimb. The negative pole of the needle was positioned through the transitional area of the lateral gastrocnemius and the TA muscle, close to the common peroneal nerve. The positive pole of the needle electrode was placed percutaneously, distal to the cathode at the ventral side of the TA muscle. When a voltage over $4 \mathrm{~V}$ was needed to obtain maximal contraction the cathode was repositioned to stimulate closer to the nerve. To prevent electrode 
displacement due to movement, muscle contraction or a combination of the two: both electrodes were fixed when positioning was optimal (maximal work with voltage $<4 \mathrm{~V}$ ) using cyanoacrylate glue.

\section{Exercise protocol}

Seventy-two rats were randomly assigned to one of 2 groups. One group $(n=36)$ performed 240 lengthening contractions on day 0 (referred to as the $L$ group) after which rats were sacrificed for muscle sampling on day $0(n=6)$, on day $4(n=6)$ and at day $15(n=6)$. The 18 remaining rats were again subjected to 240 lengthening contractions on day 15 (LL group) and sacrificed on day 15, day 19 and day 30 ( $n=6$ in every group). In the second group $(n=36)$ the same procedure was followed, however in this group the exercise session on day 0 comprised 240 shortening contractions (the $S$ group), followed by 240 lengthening contractions on day 15 (SL group). The exercise protocol and muscle sampling points are depicted in Figure 7.1.

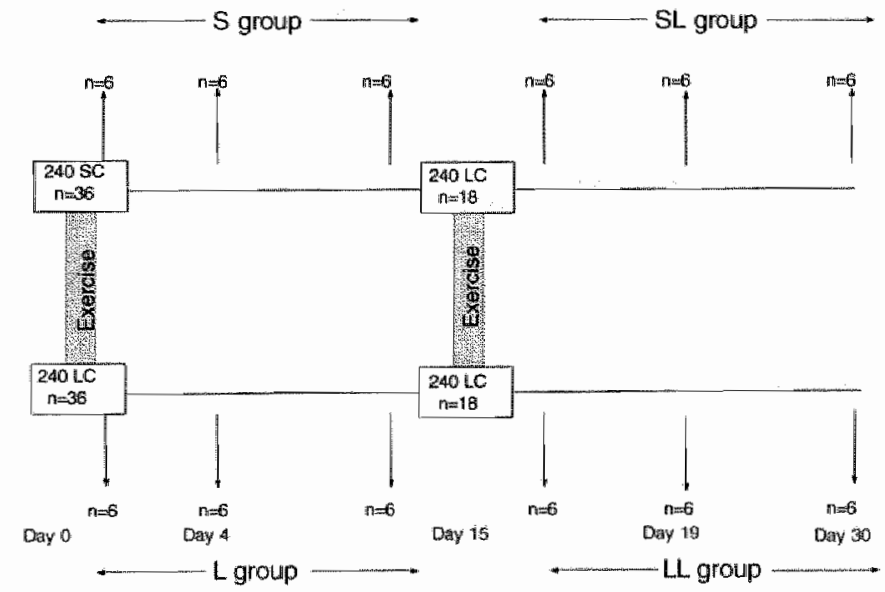

Figure 7.1: Study design. $\mathrm{SC}=$ shortening contractions, $\mathrm{LC}=$ =lengthening contractions. Muscle samples for $B$-GU activity and morphology are taken at day 0 , day 4 , betore and after exercise on day 15 , on day 19 and day 30 .

During exercise the TA and EDL muscles were unilaterally subjected to 4 consecutive exercise bouts of 60 isokinetic $\left(500^{\circ} \cdot \mathrm{s}^{-1}, 1.2 \mathrm{Lf} \cdot \mathrm{S}^{-1}\right)$ fused tetanic contractions. Muscles were forced to contract every 3 seconds for $310 \mathrm{~ms}$ using individually determined supramaximal stimulation conditions (typically $\sim 3 \mathrm{~V}, \sim 130$ $\mathrm{Hz}$ ) with $0.1 \mathrm{~ms}$ pulse trains. Sequential exercise bouts were interspaced by 5 minutes recovery.

\section{Tissue sampling and processing}

Dorsiflexor muscles were removed bilaterally and mass of the exercised muscles of rats sacrificed immediately post-exercise (day 0 and day 15) was assessed using a Sartorius 1216 MP scale. Mass of the active muscles at the time of exercise in the groups sacrificed 4 and 15 d post-exercise was calculated using a previously 
assessed relationship between body mass (BM) (g) and active muscle mass (MM) (mg) of 76 male Wistar rats ( $M M=2.4221 \cdot B M, R^{2}=0.803, p<0.0001$ ).

Only the TA muscle was processed for histological and biochemical analysis. The mid-belly region of the TA muscle was used for histological examination and mounted on a thin piece of cork with O.C.T. embedding compound (Tissue-tek ${ }^{\circledR}$, Miles Laboratories) and frozen in isopentane cooled to melting point with liquid nitrogen. The remainder of the TA was freeze-clamped using a pair of liquid-nitrogen-cooled metal tongs and B-glucuronidase (B-GU) activity (expressed as $\mu \mathrm{mol} \cdot \mathrm{s}^{-1} \cdot \mathrm{kg}^{-1}$ protein) was analyzed according to Barrett (5) from phosphate buffered water homogenates. Protein content was assessed according to Peterson (24).

Of the samples taken on day 4 and day 19 , twenty serial iransverse cryosections of $10 \mu \mathrm{m}$ were cut at $-20^{\circ} \mathrm{C}$, thaw-mounted on glass slides and stained with hematoxylin and eosin for qualitative light microscopical examination.

Statistics

Contractile work was compared by paired ( $L$ with $L L$ ) and unpaired ( $S L$ with $L$ and LL) Student t-tests with Bonferroni correction. Paired Student t-tests were performed to compare total work during successive exercise bouts within the first and second exercise session ( $S$ with $S L$ and $L$ with $L L$ ), while differences between total work during the two exercise regimes were compared using unpaired t-tests. For comparison of B-GU activity between the exercise regimes (S with $L$ and $S L$ with LL) unpaired t-tests were used while paired t-tests were employed for comparison of the exercised with the non-exercised leg. A factorial ANOVA was performed for comparison of B-GU activity on successive days, Scheffé post-hoc testing was used to localize differences. The level of significance was set at $p<0.05$. All data are presented as mean $\pm S D$, unless stated otherwise.

\section{Results}

Activity of B-GU 4 days after lengthening exercise (L, SL and $L L$ ) increased significantly compared to contralateral controls while after shortening exercise no changes were observed (Figure 7.2). At day 19, B-GU activity in the SL group increased significantly compared to controls, $S, L$ as well as compared to $L L$ (Figure 7.2). At the other timepoints B-GU activity was not significantly changed.

Muscles were extensively damaged when subjected to lengthening exercise ( $L$ and $S L$ ) (Figure 7.3). Examination of muscle samples taken after shortening exercise (S) or after the second lengthening exercise session (LL) revealed that virtually no damage occurred after shortening exercise and that damage in the LL group was less pronounced than in muscles subjected to lengthening exercise for the first time (Figure 7.3). These qualitative histological results well match the quantitative $B-G U$ results. 


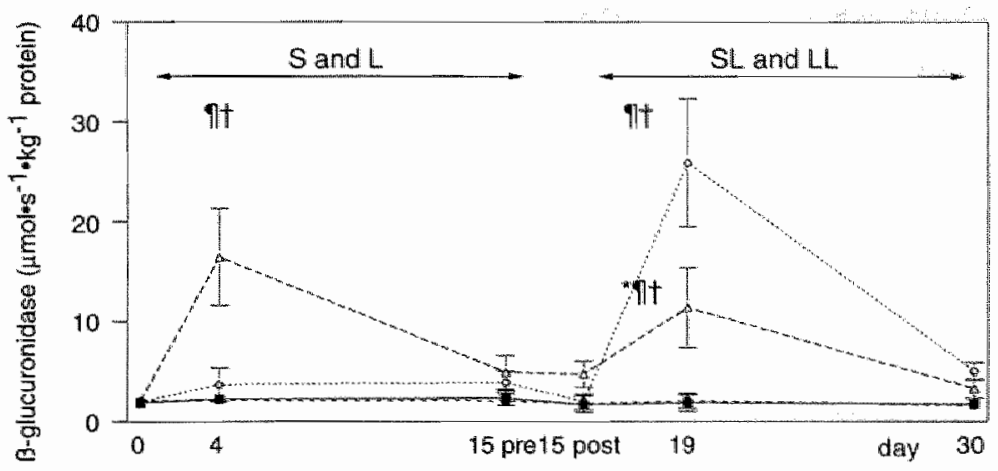

Figure 7.2: B-glucuronidase activity after the first ( $S$ and $L$ ) and the second exercise session (SL and $L L$ ). Data are presented as mean $\pm S D(n=6)$. 1) = significantly different from non-exercised control, $\dagger=$ significantly different from shortening exercise, ${ }^{*}=$ significantly different from $\mathrm{L}$ and $\mathrm{SL}$. Filled symbols refer to non-exercised muscles, open symbols refer to exercised muscles.
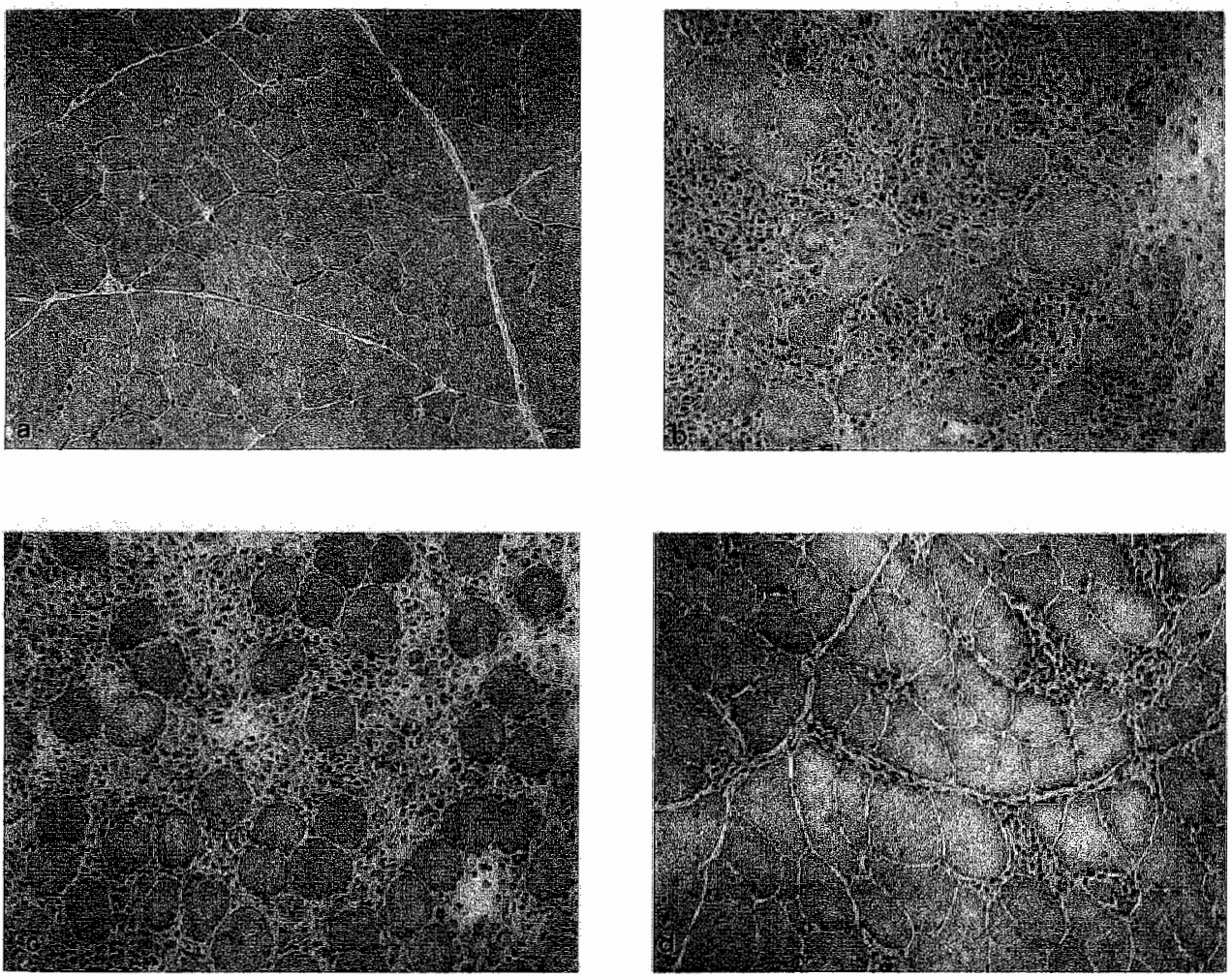

Figure 7.3: Representative photomicrographs of cross-sections of TA muscles sampled 4 days after the first and the second exercise session, stained with hematoxilin and eosin. Cross-sections shown are taken after (a) shortening exercise (group S), (b) lengthening exercise (group L), (c) shortening exercise followed by lengthening (group $\mathrm{SL}$ ) and (d) after the second lengthening exercise session (LL). 
At the onset of the study, measured mass of the exercised muscles was similar in $S$ and $L$ ( $879 \pm 28.1$ and $885 \pm 16.0 \mathrm{mg}$, respectively). After 15 days measured mass of the exercised muscles was increased significantly $(966 \pm 40.7$ in SL and $978 \pm 36.4 \mathrm{mg}$ in $\mathrm{LL}$ ) but did not differ between groups.
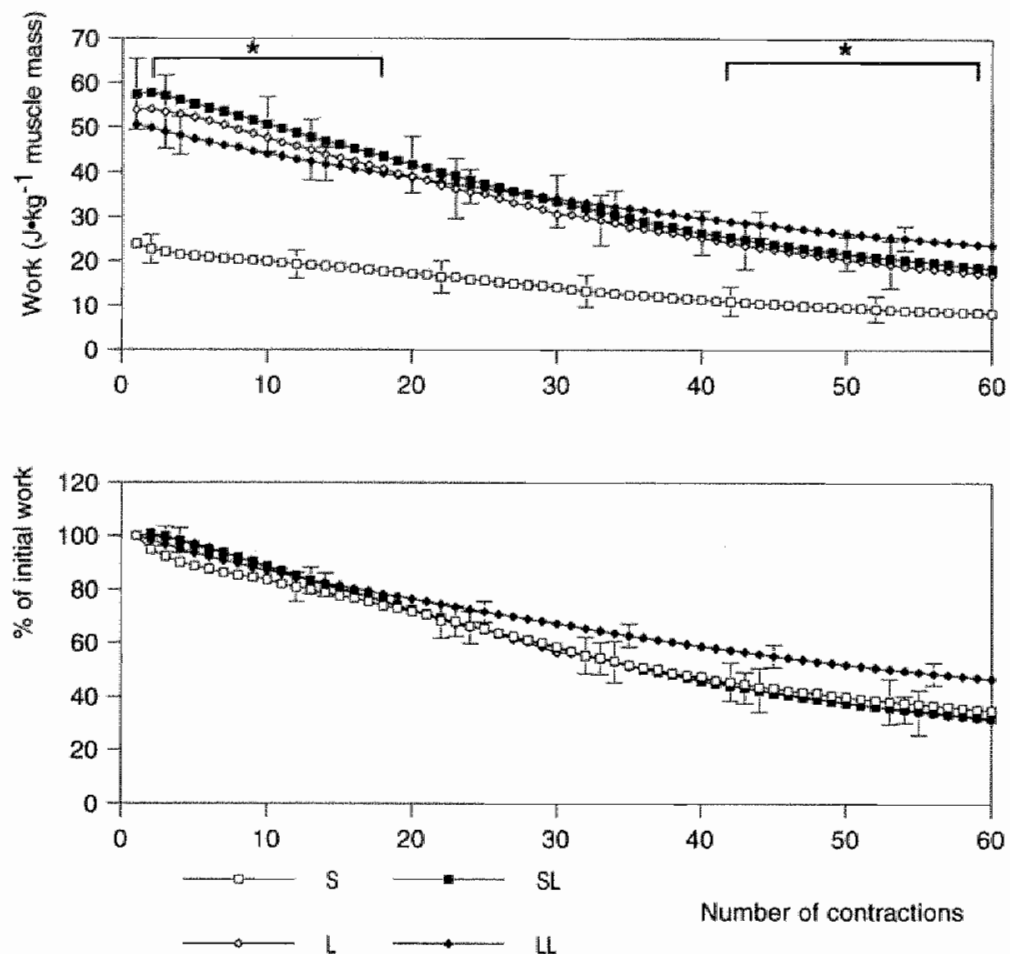

Figure 7.4: Absolute (upper panel) and relative external work (lower parnel) during every single contraction recorded during exercise bout 1 , open symbols refer to the first exercise session ( $S$ stands for shortening. $L$ stands for llengthening) while filled symbols refer to the second exercise session (SL corresponds to lengthening contractions of concentrically conditioned muscles, $\mathrm{LL}$ refers to a second session of lengthening contractions). Data are presented as mean $\pm S D(n=18)$, for illustrative purposes, standard deviations are only depicted every $10^{\text {th }}$ contraction. " denotes a significant difference ( $\left.p<0.05\right)$ when comparing $L L$ with both $L$ (contraction $4-8$ and $40-60$, respectively) and $S L$ (contraction $1-18$ and 40-60, respectively).

Contractile work during shortening exercise (S) is consistently lower than work during lengthening exercise (L, SL and $L L$ ) (Figure 7.4, upper panel). No differences in work between muscles undergaing lengthening exercise for the first time ( $L$ and $S L$ ) were observed. In contrast, muscles undergoing lengthening contractions for the second time (LL) generated a lower amount of work during the first few contractions of the first exercise bout than muscles performing lengthening exercise for the first time ( $L$ and $S L$ ), while more work was generated during the final 20 contractions of the first exercise bout in $L L$ than in $L$ and $S L$ (Figure 7.4, upper panel). 
To compare the decline in work during successive shortening and lengthening contractions, contractile work is computed relative to the amount of work performed during the first contraction (Figure 7.4, lower panel). The relative decline in work is similar in $S, L$ and $S L$ while the relative decline in work during $L L$ is lower than during $S$, $L$ or $S L$.

The total amount of work performed during successive lengthening exercise bouts is shown in figure 7.5. Except for the amount of work performed during the first exercise bout, muscles subjected to prior lengthening exercise (LL) performed significantly more work during the second lengthening exercise session than during $L$ and $S L$ (Figure 7.5 ).

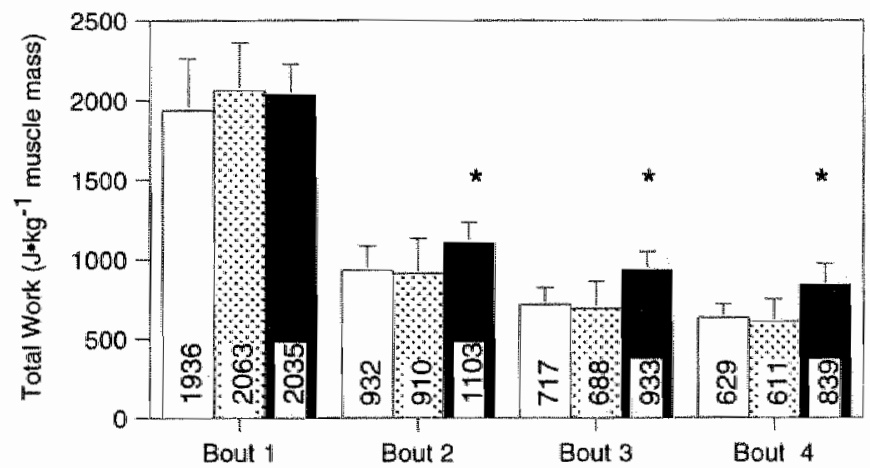

Figure 7.5: Total work during successive lengthening exercise bouts. Data are presented as mean \pm SD $(n=18) .{ }^{*}$ denotes a significant difference when comparing $L L$ with both $L$ and $S L$. Open bars represent the $L$ group, dotted bars represent the SL group and the black bars represent the $L L$ group.

\section{Discussion}

Others have shown that maximal force and fiber morphology were preserved when a damaging exercise session was repeated within 21 days after the exercise session inducing the initial injury (25). It was our aim to study the repeated bout effect with emphasis on indices of muscle damage, on contractile work during successive lengthening contractions (LC), as well as on the total amount of work performed during successive exercise bouts.

For quantification of muscle damage we have chosen to analyze Bglucuronidase (B-GU) activity, since activity of B-GU correlates highly with the histopathological state 3 and $5 \mathrm{~d}$ post-exercise $(0.746$ and 0.873 , respectively (27)).

Muscles subjected to lengthening exercise for the first time ( $L$ and $S L$ ) were extensively damaged, as indicated by their morphological appearance as well as by elevated activity of B-GU. Following shortening exercise (S), muscles appeared to have normal morphology and displayed an insignificant elevation in B-GU activity. These observations are in agreement with previous studies $(10,12,14,15$, 18). Muscles undergoing lengthening exercise for the second time (LL) demonstrated preserved muscle morphology and a blunted increase in B-GU, indicating a protective effect of prior lengthening exercise. The observation that a protective effect of a single prior exercise session is restricted to lengthening training is in line with the literature $(9,13,17)$. The protective effect of lengthening 
exercise manifests itself in improved muscle function during a second lengthening exercise session.

During the second exercise session contractile work in (SL) during lengthening exercise well matched contractile work during $L C$ in the first exercise session (L). Work recorded during the first contractions in LL was $\sim 5 \%$ less compared to muscles performing lengthening exercise for the first time ( $S L$ and $L$ ). It could be argued that the observed protective effect of prior lengthening exercise in LL (preserved muscle morphology and blunted increase in B-GU) originates from lower contractile work during the first few contractions in LL. However, after 40 lengthening contractions, contractile work in $\mathrm{LL}$ is significantly larger than in $\mathrm{L}$ or $\mathrm{SL}$. In total "muscles previously subjected to lengthening exercise (LL) generated $\sim 15 \%$ more work during the second exercise session than in the L and SL groups. It is therefore unlikely that the observed preservation of muscle morphology and blunted increase in B-GU originates from a lower work input in $L L$ compared to $L$ and SL.

Based on observations of Stevens (31) we expected relative work during successive LC to fall progressively and to a greater extent than during successive SC. Since no muscle damage was detected after SC, the decline in work during successive SC is attributed to changes in energy metabolism. The decline in relative work during successive LC most likely originates from a combination of altered energy metabolism and damage-induced contractile malfunction. Therefore, relative work (percentage decline from the first to the final contraction) during LC will decline more than during SC when only metabolic alterations are present. However, we observed that relative contractile work declined similarly during shortening $(S)$ and lengthening exercise in previously untrained muscles ( $L$ and $\mathrm{SL}$ ). This contrasts with most of the available literature showing a more pronounced decline in maximal isometric force recorded after successive LC than after successive SC $(4,17,26,29)$. However, work recorded during a contraction is essentially different from recording maximal isometric force after exercise. Since the number of sarcomeres increases following downhill running (lengthening exercise) (16), lengthening exercise-induced changes in muscle function are more accurately monitored if work is recorded over a range of motion (i.e., a range of sarcomere lengths) than if muscle function is recorded by assessment of isometric force at only a small selection of sarcomere lengths. The observation that relative work declines similarly during high-intensity shortening and lengthening exercise in previously untrained muscles appears to be a novel and unexplained finding.

Total work performed during $L C$ in the first exercise bout was identical for $L$, $\mathrm{LL}$ and SL (Figure 7.4), while during the $2^{\text {nd }}, 3^{\text {rd }}$ and $4^{\text {th }}$ exercise bout total work was consistently higher in LL than in $S L$ or $L$. This observation indicates that muscles previously subjected to LC are more resistant to the stress posed upon them during lengthening exercise and are capable to generate more work during a second lengthening exercise session. Although increased maximal isometric force following lengthening training has been reported previously (e.g. 4, 17, 26, 29), the present study is the first to conclusively show that muscles subjected to damaging exercise for the second time are able to generate more work (i.e., are less fatiguable) during a second session of LC. Since muscles were exercised by 
supramaximal electrical stimulation (fused tetanic contractions), thus surpassing the central nerve system, the potential bias of remained muscle soreness is ruled out in the present study.

Whether the adaptive response observed here is of metabolic or mechanical origin can not be elucidated by the present data-set. It has been hypothesized that the adaptive effect after one session of lengthening contractions originates from an increased number of sarcomeres in series (19). Consequently, when using the same range of motion, a second lengthening exercise session will not, or to a lesser extent, involve the descending limb of the force-length curve. Since Morgan suggested that muscle damage is initiated by "popping" of sarcomeres at the descending limb of the force-length curve (19), muscle damage will be diminished if the number of sarcomeres in series increases. An increased number of sarcomeres in series instigates a shift in the force-length relationship of the affected muscle and may induce an increase in contractile work over the same range of motion. When force transmitting proteins are strengthened (11) upon damaging exercise, it is to be expected that muscles previously subjected to lengthening exercise demonstrate improved force transmission (23) and are therefore able to cope with higher stresses during a second lengthening exercise session. Consequently, higher work values can be maintained. In support of Morgan's hypothesis are reports showing that the mean number of sarcomeres per fiber is greater after downhill running than after uphill running (16). If sarcomero-genesis increases in the exercise model used in the present study, sarcomere lengths at the onset of rotation will be decreased, subsequently peak torque will occur at longer muscle lengths (i.e., relatively more plantarflexion) and contractile work will increase, as observed in the present study. Of the data reported here, preserved muscle morphology and deciined B-GU activity following lengthening training indirectly support Morgan's hypothesis (19).

In conclusion, it is shown that muscle damage induced by lengthening exercise was alleviated when conducted $15 \mathrm{~d}$ after an identical session of lengthening exercise. The adaptive response to lengthening exercise is functionaliy reflected in a less rapid and less severe decline in contractile work and in an increased total amount of work performed (i.e., decreased fatiguability) during a second lengthening exercise session.

\section{Acknowledgment}

We gratefully acknowledge the financial support by foundation "De Drie Lichten", permitting construction of the set-up described in here. 


\section{Dew wanes}

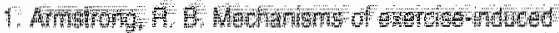

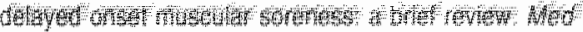

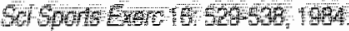

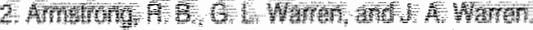

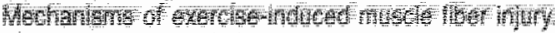

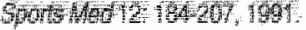

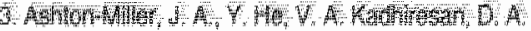

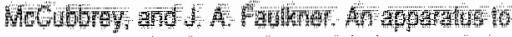

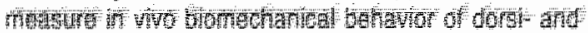

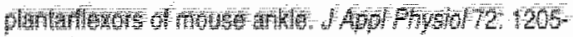
$\sqrt[1]{162}$

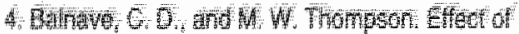

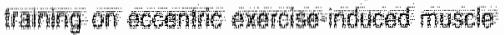

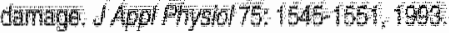

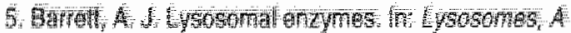

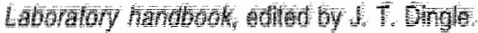

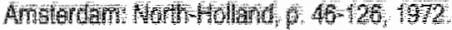

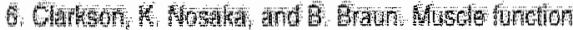

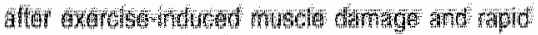

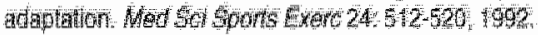

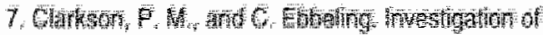

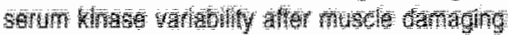

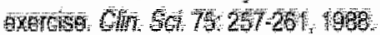

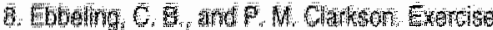

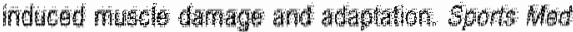
$7,07.24$

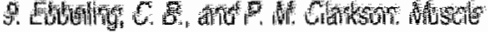
adaptation prior to recovery following eccentric exercise, Eur J Appl Physiol 60: 26-31, 1990.

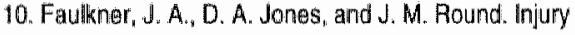
to skeletal muscles of mice by forced lengthening during contractions. Q J Exp Physio/ 74: 661-670, 1989.

11. Friden, J. Exercise-induced muscle soreness; a qualitative and quantitative study of human muscle morphology and function. Umed Unversity Medical Dissertations New Series, 1983.

12. Hesselink, M. K. C, H. Kuipers, P. Geurten, and H. wan Straaten. Structural muscle damage and muscle strenglh after incremental number af isometric and forced lengthening contractions. IMuscle Res Celf Motil 17: 335-341, 1996.

13. Kontulainen, J, and W. Vinko. Swirnming exercise and skeletal muscle damage in mice. Med Sci Res 20: $413-415,1992$.

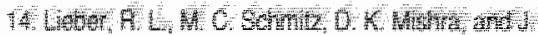

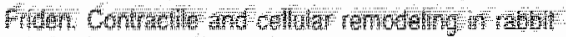

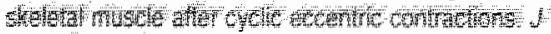

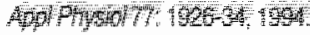

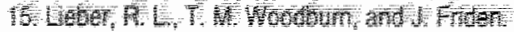

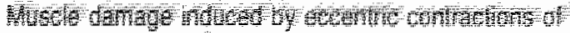

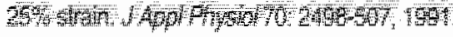

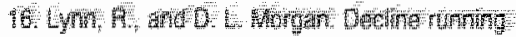

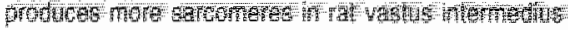

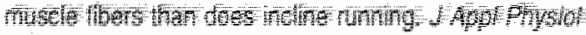

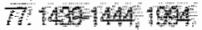

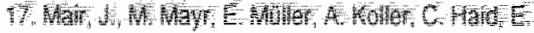

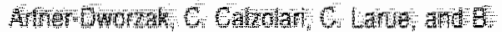

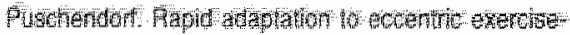

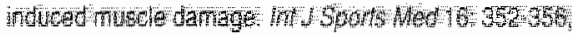
15

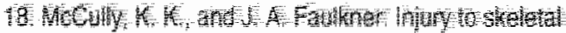

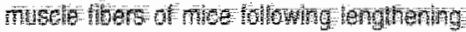

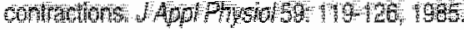

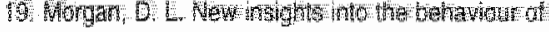

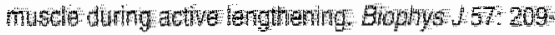
27,199

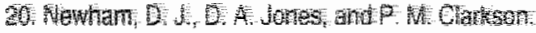

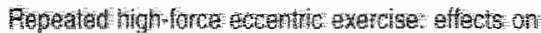

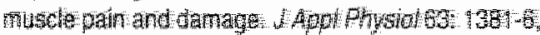
tad.

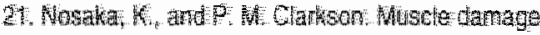

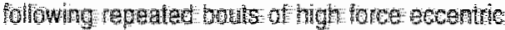
exercise Me

22. Nosaka, K., P. M. Clarkson, M. E. McGuiggin, and J. M. Byrne. Time course of muscle adaptation after high force eccentric exercise. Eur J Appl Physiol 63: 70-76 1991.

23. Patel, T. J., and A. L. Leiber. Force Transmission in Skeletal Muscle: Hrom Actomyosin to External Tendons In: Exercise and Sport Sciences Reviews, edited by J. O. Holloszy. St. Louis: Williams \& Wilkins, p. 321-363. 1997

24. Peterson, G. L. Simplification of protein assay of Lowry et al. which is more general applicable. Anal Biochem 83: 346-356, 1977 .

25. Sacco, P., and D. A. Jones. The protective effect of damaging eccentric exercise against repeated bouts of exercise in the mouse tibialis anterior muscle. Experimental physiology 77: 757-760, 1992 
26. Sacco, P. D. A. Jones, J. R. T. Dick, and G. Vrbova. Contractile properties and susceptibility to exerciseinduced damage of normal and mox mouse tibialis anterior muscle. Clin Sci 82: 227-36, 1992.

27. Salminen, $A_{x_{x}}$ and $M$. Kihlström. Lysosomal changes in mouse skeletal muscle during the repair of exercise injuries. Muscle and Nerve 8: 269-279, 1985.

28. Schwane, J. A., and R. B. Arrnstrong. Effect of training on skeletal muscle injury from downhill running in rats. I Appl Physiol 55: 969-75, 1983.

29. Smith, L. L., M. G. Fuimer, D. Holbert, M. R. MCCammon "J. A. Houmard, D. D. Frazer, E. Nsien, and R. G. Israel, The impact of a repeated bout of eccentric exercise on muscular strength, muscle soreness and creatine kinase. Br J Sp Med 28: 267-271, 1994.

30. Stauber, W. T. Eccentric action of muscles: Physiology, injury, and adaptation. Exerc Sports Sci Rew 17: 157-185, 1989.

31. Stevens, E. D. Elfect of phase of stimulation on acute damage caused by eccentric contractions in mouse soleus muscile. W' Appl Physiol 80: 1958-1962, 1996. 



\section{Changes in the Expression of Myofibrillar and Cytoskeletal Proteins Following Shortening and Repeated Lengthening Exercise}

M.K.C. Hesselink, H. Kuipers, H.A. Keizer, M.R. Drost, H.J.H. Kuijpers ${ }^{1}$, F.C.S. Ramaekers ${ }^{1}$

1Department of Molecular Cell Biology \& Genetics, Maastricht University. 


\section{Abstract}

In the present study the effect of (repeated) lengthening exercise on the expression of myofibrillar and cytoskeletal proteins was studied in rat skeletal muscle. Tibialis anterior muscles were subjected to 240 contractions in one or two exercise sessions. The first exercise session consisted of 240 shortening (S) or lengthening (L) contractions. Muscles were excised 4 days after the first exercise session or 4 days after a second exercise session to which the rats were subjected 15 days later. During the second exercise session only lengthening contractions were employed, resulting in SL and LL grcups. Contralateral, non-exercised muscles served as controls. Protein analysis was performed on muscle sections by oneand two-dimensional gel electrophoresis. Western blotting was done with antibodies against desmin and vimentin. The protein expression pattern was unaffected by shortening exercise. However, re-expression of the intermediate filament protein vimentin next to desmin was observed after a single lengthening exercise session, indicating remodeling of the cytoskeletal lattice. This remodeling is accelerated in muscles previously subjected to lengthening exercise. After single $(L, S L)$ and repeated lengthening (LL) exercise, a $94 \mathrm{kD}$ protein, hypothesized to represent $\alpha$-actinin, is largely lost from the insoluble fraction of the muscle cells. In the low molecular weight region the major alterations were observed amongst the 14-20 kD proteins, likely to represent myosin light chains (MLC) 1,2 and 3 . Following lengthening exercise in particular, MLC-3 becomes less abundantly expressed. It is concluded that both the intermediate filament network as well as the sarcomeric structure is remodeled and reorganized following lengthening exercise and that a single shortening exercise session does not affect expression of myofibrillar and, cytoskeletal proteins. 


\section{Introduction}

Lengthening exercise induces damage to the exercised muscles (see for example $1,5,7,8,17)$. Muscle damage comprises distortion of the contractile apparatus; cytoskeletal damage, extracellular matrix abnormalities (9) and immediate postexercise loss of immuno-staining of the lateral Z-disc connector desmin $(10,19)$.

Physical exercise on a regular basis induces transient adaptations in muscle $(2,12)$, which permit the muscle to better cope with increased demands of work. In contrast to shortening exercise, lengthening exercised muscles demonstrate a rapid and long lasting protection against damage induced by lengthening contractions, which is detected already after a single exercise session. The adaptive response after lengthening exercise is functionally reflected in the capability to generate more work during (11) or more force after $(3,28)$ a second lengthening exercise session and is referred to as the 'repeated bout effect'.

The mechanism underlying the repeated bout effect is still incompletely understood. Potential molecular sites of adaptation are the myofibrillar and cytoskeletal proteins. So far, the majority of research on altered protein expression after muscle damage has focused on total protein synthesis and degradation patterns $(20,34,35)$, without differentiating between myofibrillar, cytoskeletal and soluble proteins.

In skeletal muscle both endo- and exosarcomeric cytoskeletal proteins connected to the contractile proteins play a functional role in longitudinal and transverse force transmission (26). Improved or more efficient force transmission in skeletal muscle may contribute to the capability to generate more work during a second lengthening exercise session. As a consequence, changes in the expression of endo- and exosarcomeric muscle proteins may correlate with structural adaptation to damaging lengthening exercise.

Therefore, the present study was conducted to monitor myofibrillar and cytoskeletal protein expression after shortening and repeated lengthening exercise. The effect of repeated lengthening exercise was studied by superimposing a lengthening exercise session $15 d$ after the first exercise session comprising either shortening or lengthening contractions. It was hypothesized that, in contrast to lengthening exercise, a single shortening exercise session would not affect expression of myofibrillar and cytoskeletal proteins. Furthermore, it was hypothesized that following a second lengthening exercise session alterations in protein expression would be less prominent than after the first lengthening exercise session.

\section{Materials and methods}

\section{Animals}

Tibialis anterior (TA) muscles of the hindlimbs of mature male Wistar rats (Charles River), aged 12 weeks at the onset of the study, were studied following lengthening and shortening exercise. Exercise and muscle dissection were performed under pentobarbital anesthesia (Narcovet ${ }^{(\ll)} 1.0 \mathrm{ml} / \mathrm{kg}^{-1}$ body weight, i.p. injection) after short-term (less than $20 \mathrm{~s}$ ) $\mathrm{CO}_{2}$ sedation. Four days after the final exercise session muscles were dissected and rats were sacrificed by cervical dislocation. All experimental procedures were approved by the Institutional Animal Care and Use 
Committee of the Maastricht University and complied with the principles of laboratory animal care.

\section{Exercise protocol}

Sixteen rats were randomly assigned to one of 4 exercise regimes $(n=4$ in every group). Rats were subjected to either 240 shortening or lengthening contractions (referred to as the $S$ and $L$ group, respectively), followed by 240 lengthening contractions in 8 rats $15 d$ after the first session, regardless of the type of exercise performed during the initial exercise session. Rats subjected to lengthening exercise twice are referred to as $L L$, rats subjected to shortening exercise fallowed by lengthening exercise are referred to as SL. Contralateral TA muscles were used as non-exercised controls.

Muscles were dissected and rats were sacrificed 4 days after the first exercise session (day 4) or 4 clays after the second exercise session (day 19). The exercise protocol and successive sampling points are schematically represented in Figure 8.1.

$\mathrm{S}$

$S L$

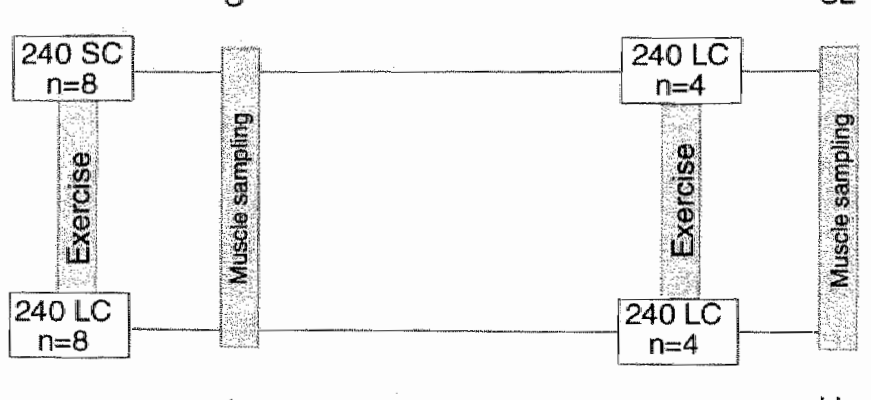

Day 0 Day 4

Day 15 Day 19

Figure 8. 1: Experimental protocol: rats were unilaterally subjected to either 240 shortening $(n=8)$ or 240 lengthening contractions $(n=8)$ on day 0 . On day 4 muscles were dissected after shortening $(n=4)$ and lengthening ( $n=4)$ exercise. The remaining rats were subjected to lengthening exercise on day 15 and muscles were dissected on day 19 .

During exercise, rat hindlimb dorsiflexar muscles were unilaterally subjected to 4 successive exercise bouts of 60 isokinetic $\left(500^{\circ} \cdot \mathrm{s}^{-1}, 1.2 \mathrm{Lf} \cdot \mathrm{s}^{-1}\right)$ fused tetanic contractions in a custom-built rat dynamometer described in detail elsewhere (11). This rat dynamometer permits accurate and valid assessment of muscle contractile work during lengthening and shortening (11). Using percutaneous needle electrodes, muscles were contracted every 3 seconds for $310 \mathrm{~ms}$ with individually determined supramaximal stimulation conditions (typically $\sim 3 \mathrm{~V}_{v} \sim 130 \mathrm{~Hz}$ ). The negative pole of the electrode was positioned through the transitional area of the lateral gastrocnemius and the TA muscle, close to the common peroneal nerve. The positive pole of the needle electrode was placed percutaneously, distal to the cathode at the ventral side of the TA muscle. Sequential exercise bouts were interspaced by 5 minutes of recovery. 


\section{Tissue sampling}

Dorsiflexor muscles were removed bilaterally. The midbelly region of the TA muscle was sampled and trozen in isopentane cooled to melting point with louid nitrogen. Semt-thick untixed transverse cryosections (10 $\mathrm{mm}$ thick) were cut at $-20^{\circ} \mathrm{C}_{3}$ thaw-mounted on glass stides and routinely stained with hematoxyln and eosin for ligh microscopical examination. Approximately 10-15 sections of 20 wh thick were collected and stored at $-80^{\circ} \mathrm{C}$ before being processed for gel electrophoresis.

Tissue preparation and gel electrophoresis

Frozen sections ( $20 \mathrm{fm}$ thick) were suspended in phosphate buffered saline (PBS). $\mathrm{pH} 7.4$, containing $1 \%$ Triton X-100 (BDH. Poole England) and stirred for 5 min at $0 \circ \mathrm{C}$. After centrifugation the pellet was resuspended in approximately 3 volumes of sample buffer containing sodium dodecyl sulfate (SDS; Bio-Rad Laboratories, Hercules, Californa) and bolled for $4 \mathrm{~min}$.

One dimensional SDS-polyacrylamide gel electrophoresis (1D-SDS-PACE) was performed according to Laemmli (15) using gels containing either $7.5 \%$ or $13 \%$ acrylamide (Bio-Rad Laboratories, Hercules, Calfornia) and $0.1 \%$ SDS. Gess were run for approximately $45 \mathrm{~min}$ at $200 \mathrm{~V}$ in the Mini-Protean II system (Bio-Rad Laboratories, Hercules, California). For comparison, a Triton $X-100$ extracted cell pellet of a NCl-H125 tung cancer cell culture was used.

For further characterization of the proteins identified in 1D-SDS-PAGE, selected samples were subjected to two-dimensional polyacrylamide get electrophoresis (2D-PAGE), which was performed on the Triton X-100 extracted cell pellets as described by O'Farrell et al. (25). For comparison of the quantity of protein loaded onto each lane, gels were stained with Page Blue 83 (Fiuka, Brunschwig, Germany).

Western blotting was performed essentially according to Towbin et al. (32). using monoclonal antibodies against desmin (RD301) (6) and vimentin (RV202) (27). Proteins were transferred to nitrocellulose membranes (BAB5, Schleicher and Schüll, Dassel Germany) by electrophoresis for $1 \mathrm{~h}$ at $100 \mathrm{~V}$ using the Mini TransBlot Cell (Bio-Rad Laboratories). The sheets were air-dried and blocked for $1 \mathrm{~h}$ at room temperature in buffer containing $10 \mathrm{mM}$ Tris- $\mathrm{HCl} \mathrm{pH} \mathrm{7.6,0.35} \mathrm{M} \mathrm{NaCl}$ and $3 \%$ bovine serum albumin (BSA; Sigma). Thereafter, the blots were incubated with the primary antibodies in appropriate dilutions for 60 minutes at room temperature in buffer containing $10 \mathrm{mM}$ Tris- $\mathrm{HCl}$ pH $7.6,0.15 \mathrm{M} \mathrm{NaCl}, 0.3 \% \mathrm{BSA}, 1 \%$ Triton $\mathrm{X}$ $100,0.5 \%$ sodium deoxycholate (DOC; Merck) and $0.1 \%$ SDS (Merck). Subsequently, the blots were washed 3 times in PBS containing $0.5 \%$ Triton $X-100$ and incubated for 60 minutes at room temperature with a peroxidase conjugated rabbit anti mouse IgG antibody (DAKO A/S; Glostrup, Denmark), diluted 1:400 in PBS with $0.5 \%$ BSA and $0.5 \%$ Triton X-100. Finally, the blots were extensively washed in PBS with $0.5 \%$ Triton $X-100$ and peroxidase activity was detected using an enhanced chemiluminesence kit (Amersham Life Science, Amersham international, Bucks, United Kingdom). 


\section{Results}

\section{Histological studies}

Quantitative examination of sections stained with hematoxilin and eosin revealed that muscles were extensively damaged following the first lengthening exercise session (L) as indicated by central nuclei, infiltration with monocytes and fiber necrosis (Fig 8.2a). Examination of muscle samples taken after shortening exercise (S) or after the second lengthening exercise (LL) session showed that virtually no damage occurred after shortening exercise (Fig 8.2b), and that damage in the LL group (Fig $8.2 \mathrm{c}$ ) was less prominent than in muscles in the SL group (Fig 8.2d).
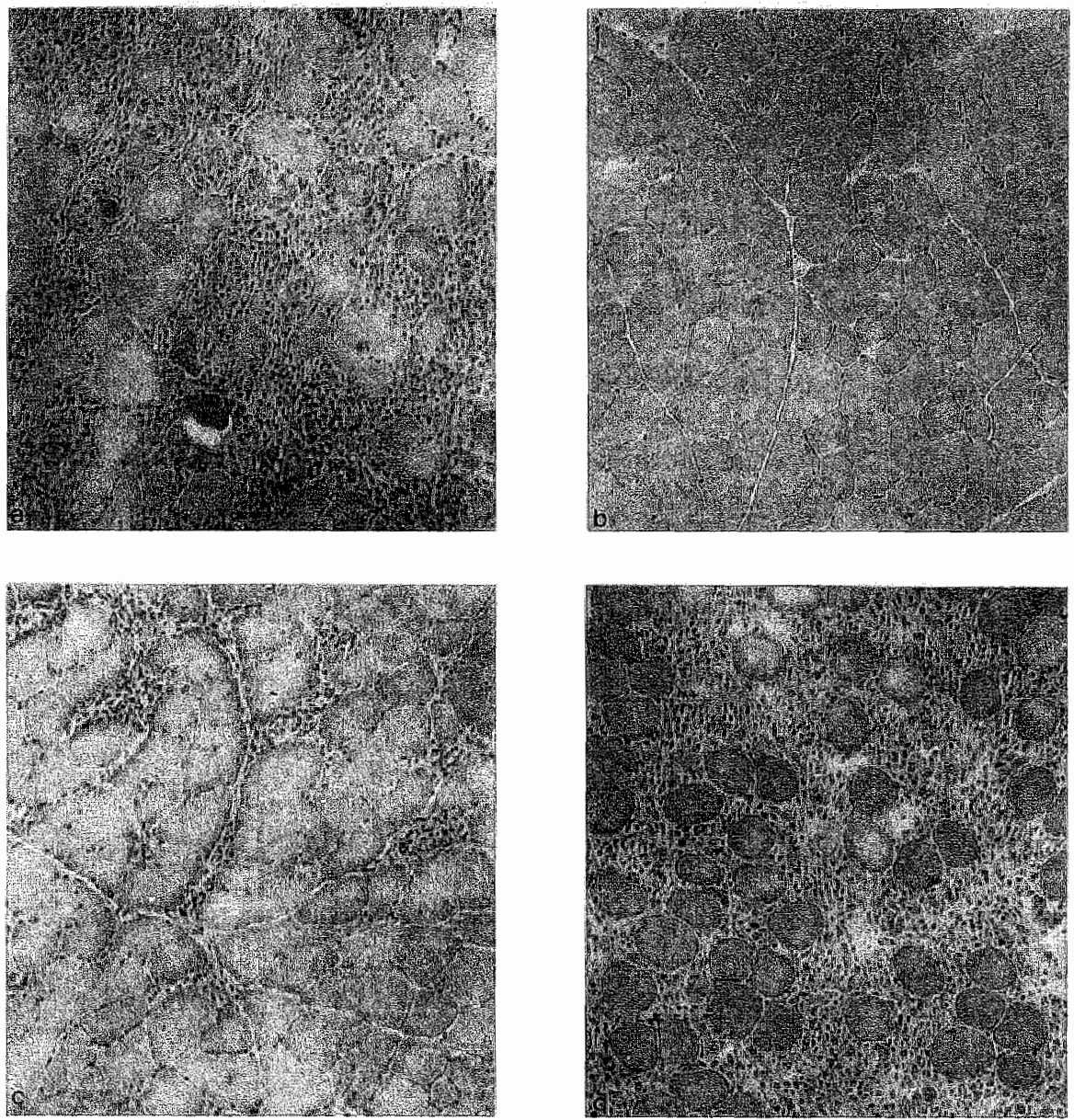

Figure 8.2: Representative cross sections after $L, S, L L$ and $S L$ are depicted in $a, b, c$ en $d$, respectively. Note the extensive muscle damage observed after a single lengthening exercise session ( $a$ and $d$ ), while after a second lengthening exercise session muscle damage was alleviated (c). After shortening exercise darnage was virtually absent (b). 


\section{One-dimensional protein separation}

Representative protein samples of exercised muscles from every group $(L, S, L L$ and SL), as well as from a contralateral control sample (C) are shown in 1D-SDSPAGE in figure $8.3 a$ and $b$. The left panel (a) shows results of $13 \%$ polyacrylamide gels to allow the analysis of low-molecular weight proteins, while the right panel (b) shows results from $7.5 \%$ polyacrylamide gels, allowing a better separation of highmolecular weight proteins.

The major differences between exercised and non-exercised muscles were observed in 2 regions in these gels, which are indicated by arrows and the bracket in figures $8.3 \mathrm{a}$ and $8.3 \mathrm{~b}$. In the $55-58 \mathrm{kD}$ region a single band was observed in samples from the control $(C)$ and $S$ groups, while in $L, S L$ and $L L$ groups intensified doublets were present. Distinct differences were also observed in the low molecular weight region (14-20 kD) between $C$ and $S$ group on the one hand and between L, SL and LL on the other. In the $C$ and $S$ group only one major protein band was observed between 14 and $20 \mathrm{kD}$, while the $\mathrm{L}, \mathrm{SL}$ and $\mathrm{LL}$ groups showed additional protein bands in this molecular weight region. When compared to the protein pattern of $\mathrm{NCl}-\mathrm{H} 125$ cell cultures, these four additional bands seem to co-migrate with the protein bands recognized as the histones (Fig 8.3c).

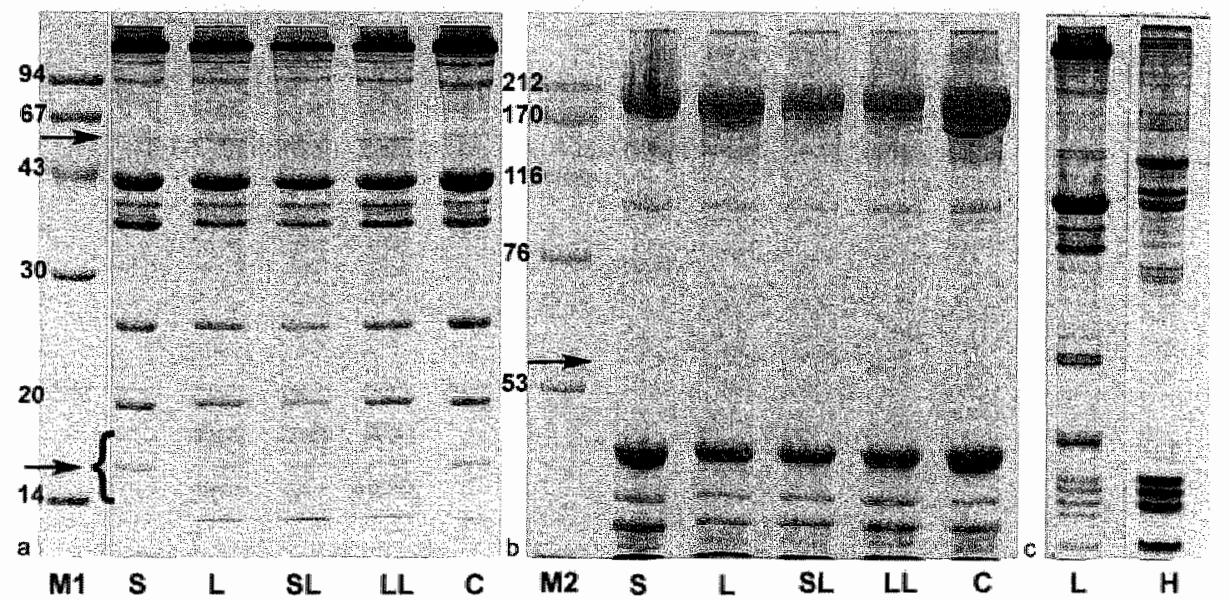

Figure 8.3: Coomassie brilliant blue staining of one-dimensional SDS-gels of Triton X-100 extracted muscle samples. Panel a depicts results from a 13\% polyacrylamide gel, while panel b shows results from $7.5 \%$ gels. Lane M1 contains the low molecular weight protein markers, lane M2 contains the high molecular protein markers, $C$ represents a non-exercised control muscle, $S, L, S L$ and $L L$ contain samples from exercised muscles. Panel c contains a sample from the $\mathrm{NCl}-\mathrm{H} 125$ cell line $(\mathrm{H})$, and a sample from the $\mathrm{L}$ group (L).

\section{Two-dimensional protein separation}

Results of 2D-PAGE of selected samples of the $S$ and $L$ group are depicted in figure 8.4, with arrows pointing to the major differences in their protein patterns. Again two different gel types were used to obtain optimal separation of both the proteins in the low (Fig 8.4a and b) and high (Fig 8.4c and d) molecular weight 
region. On basis of their relative position in the gel, several proteins, such as actin (A), desmin (D), vimentin (V) and tropomyosin (T), can be easily identified.
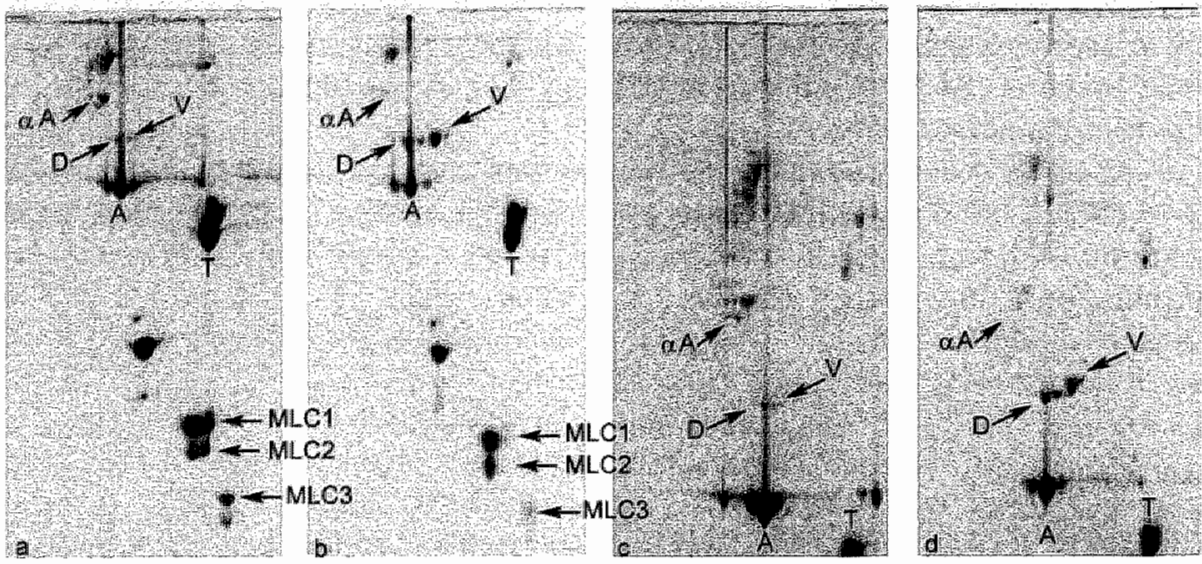

Figure 8.4: Two-dimensional gel analysis, isoelectric focusing was from left (basic) to right (alkalic), SDSPAGE was from top to battom. Results from $S$ and $L$ are shown in a $13 \%$ polyacrylamide gel in panel a and $b$, respectively. Panel $c$ and $d$ show the same samples run on a $7.5 \%$ gel. $\alpha-A$ stands for alpha actinin, $D$ for desmin, $V$ for vimentin, $A$ for actin, $T$ for both isoforms of tropomyosin, and MLC1, MLC2 and MLC3 for the myosin light chain isoforms. Identification of these proteins was based on comparison of estimated and calculated molecular weight and isoelectric $\mathrm{pH}(\mathrm{pl})$.

\begin{tabular}{|c|c|c|c|c|c|}
\hline \multirow[b]{2}{*}{ Numiber } & \multirow[b]{2}{*}{ Protein } & \multicolumn{2}{|c|}{ Calculated } & \multicolumn{2}{|c|}{ Corresponding spot in 2D gel } \\
\hline & & Mw & $\mathrm{pl}$ & Mw & $\mathrm{pl}$ \\
\hline P35609t & $\alpha$-actinin ${ }^{*}$ & 96 & 5.5 & + & + \\
\hline P31000 & vimentin* & 54 & 5.1 & \# & + \\
\hline P48675 & desmin" & 53 & 5.2 & + & + \\
\hline P02568 & $\operatorname{actin}^{*}$ & 4 & 52 & + & + \\
\hline P04692 & Iropomyosin $\alpha^{*}$ & 39 & 4.7 & + & + \\
\hline$P 02560$ & troponyosin $\beta^{*}$ & 33 & 4.7 & + & + \\
\hline P09739 & troponin $T$ & $3 \pi$ & 62 & - & - \\
\hline$P 02600$ & myosin light chain $1^{*}$ & $2 \|$ & 50 & + & + \\
\hline PO4466 & myosin light chain $2^{*}$ & 19 & 4.8 & + & + \\
\hline P02595 & troponim $\mathrm{C}$ & 18 & 4.1 & 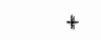 & - \\
\hline $\mathrm{P} 02601$ & myosin light chain $3^{*}$ & 16.5 & 4.6 & $*$ & + \\
\hline $\mathrm{P} 27768$ & troponim 1 & $2 \|$ & 8.9 & + & - \\
\hline
\end{tabular}

Table 8.1: Calculated MW and pl of rat skeletal muscle proteins and the presence of a corresponding spot in 2D gels (+ stands for present, while - means absent). The numbers in the first collumn refer to the protein numbers used in the Swiss protein database, the numbers marked with $\dagger$ represent proteins not characterized for rat. In these cases the data of the human isoform are given.

Table 8.1 summarizes the molecular weight and isoelectric $\mathrm{pH}$ characteristics of the major proteins observed in these gels. Spots present in 2Dgels with a molecular weight and isoelectric $\mathrm{pH}$ corresponding with the molecular weight and isoelectric $\mathrm{pH}$ from the Swiss protein database (30) are marked with an asterisk. 
In the 55-58 kD region, both gel types (1 and 2D-analysis) very clearly show that vimentin (V) is co-expressed with desmin (D) in the $L$ group but not in the $S$ group (compare Fig $8.4 \mathrm{a}$ and $\mathrm{c}$ with Fig. $8.4 \mathrm{~b}$ and d). The amount of vimentin in the $L$ group seems to exceed that of desmin.

The four protein bands that were observed in the low molecular weight region of 1D-SDS-PAGE of all L-groups but not in the $\mathrm{C}$ and $\mathrm{S}$ groups (see Fig 8.3a), are not detected in 2D-PAGE (compare the L sample in Fig 8.3a with Fig $8.4 \mathrm{~b})$. This indicates that these proteins do not penetrate the isoelectric focusing gel used for 2D-PAGE, and therefore have a high isoelectric $\mathrm{pH}$.

In the low molecular weight region some differences can however be observed when comparing the 2D-PAGE of the $S$ and the $L$ group. Isoforms of proteins that most likely represent myosin light chains 1 and 2 (MLC1 and MLC2) are present in the $S$ group but not in the $L$ group, showing only one spot for these two proteins. Furthermore, quantitative differences can be observed for the protein spot identified as myosin light chain 3 (MLC3), which is prominently present in the $C$ and the $S$ group, but virtually absent in the $L$ group.

Another reproducible difference between the $S$ and the $L$ group is observed in the high molecular weight region of the gels. In the $94 \mathrm{kD}$ region a protein band most likely corresponding to $\alpha$-actinin $(\alpha-A)$ is prominently present in the $L$ group gels, but virtually absent in the $S$ group (compare Fig $8.4 \mathrm{a}$ and $\mathrm{c}$ with Fig $8.4 \mathrm{~b}$ and d).

Furthermore, several other, as yet unidentified differences, between the $\mathrm{S}$ and the $L$ group were observed in the high molecular weight (100-250 kD) region.

\section{Western Blotting}

Based on the results of 1 and 2D-protein analysis, western blotting was performed with antibodies against desmin and vimentin. The results shown in figure 5 demonstrate that desmin is present in all samples and was most prominent in $\mathrm{LL}$ (Fig 8.5a), while vimentin, which is absent in the $C$ and $S$ group, becomes abundantly expressed in the L and SL group, and to a somewhat lesser extent also in the LL group (Fig B.5b).
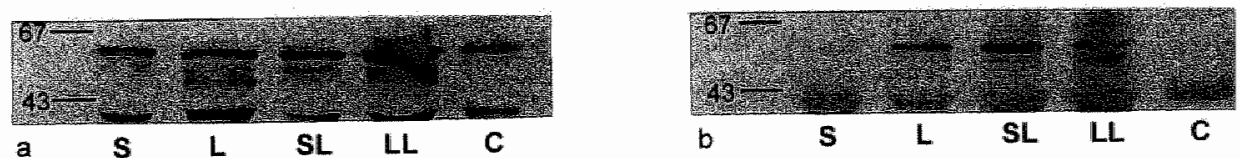

Figure 8.5: Western blotting of muscle extracts from different exercise protocols. Monoclonal antibodies against desmin (8.5a) and vimentin (8.5b) have been used.

\section{Discussion}

To study the involvement of the cytoskeleton and the contractile apparatus in the adaptation to lengthening exercise, the expression of cytoskeletal and myofibrillar proteins in rat skeletal muscle was investigated by gel electrophoresis and immunoblotting.

In skeletal muscle, actin is connected to $Z$-discs by $\alpha$-actinin, permitting longitudinal force transmission. Force transmission of myotibrils to the extracellular 
space is accomplished for by structures referred to as costameres. In vitro binding of $\alpha$-actinin to proteins present in costameres has raised the hypothesis that $\alpha$ actinin plays a functional role in lateral force transmission as well (26). Our twodimensional gel analysis revealed that after shortening exercise a protein with an isoelectric $\mathrm{pH}$ and a molecular weight corresponding to $\alpha$-actinin was present, which was largely lost after lengthening exercise. These findings may indicate that expression of $\alpha$-actinin is (transiently) depressed in muscles damaged by lengthening exercise, thus hampering force transmission. Consequently, muscle force will be reduced in seriously damaged muscles, as has previously been observed $(22,23)$. During recovery from muscle damage, redistribution and expression of $\alpha$-actinin may be such that force transmission is improved and consequently muscle force production is more efficient. However, immunoblotting is needed to provide conclusive data on expression levels of $\alpha$-actinin following shortening and repeated lengthening exercise.

In mature skeletal muscle, desmin is the major intermediate filament protein (16). In contrast with reports in the literature showing loss of desmin staining within 15 min after cyclic eccentric exercise (19), desmin was present 4 d after lengthening exercise in the present study. An explanation for these apparently conflicting results may be that following lengthening exercise desmin is transiently down-regulated immediately after exercise, and is restored to control values within $4 \mathrm{~d}$ after lengthening exercise. This is supported by our previous notion that immediate loss of desmin immunostaining occurs after lengthening and not after shortening exercise (13). Four days after lengthening exercise, however, again numerous intense desmin positive myotubes of small diameter were present (13).

During early muscle cell development, desmin is co-expressed with vimentin (14) after which the concentration of desmin gradually increases, while expression of vimentin is lost $(29,31)$. In the present study, we could not detect vimentin in TA muscle of adult Wistar rats, neither in non-exercised control samples nor $4 \mathrm{~d}$ after shortening exercise. However, 4 days after lengthening exercise vimentin was reexpressed and was found in these muscle cells together with desmin.

The relevance of desmin in creating and/or maintaining the integrity of myofibrillar interconnections is emphasized by a loss of Z-line registration, abnormal location of nuclei and an abnormal appearance of mitochondria in transgenic desmin null mice (24). Maintenance of the intermediate fillament network is a dynamic process and secondary proteolysis of the intermediate filament proteins is hypothesized to occur in preparation for muscle repair and remodeling $(18,19)$. The present study supports this hypothesis. The co-expression of desmin with vimentin after lengthening exercilse indicates repair and remodeling of the intermediate filament network, dlamaged by the lengthening exercise "similar to the situation observed during early myogenesis (14).

Reorganization of the intermediate filament network during repair and remodeling may strengthen the myofibrillar interconnections and consequently improve force transmission during a second lengthening exercise session. We previously observed that during a second lengthening exercise session more work was produced than during the first session, using identical experimental conditions 
(11), possibly reflecting improved force transmission. Following a second lengthening exercise session, vimentin was less abundantly expressed than after the first lengthening exercise session. This observation may indicate that after a second lengthening exercise session muscle repair and remodeling is accelerated compared to the first lengthening exercise session.

The low molecular weight proteins observed after shortening exercise are likely to represent myosin light chain 1,2 and $3(4,30)$. In skeletal muscle the myosin light chain isoforms have a putative role in regulating contractile speed during the power stroke (21). Phosphorylation of myosin light chains preconditions rapid force development (33). Phosphorylated isoforms of proteins possess a slightly different $\mathrm{pl}$ with a similar molecular weight, as compared to the parent protein. The two double spots observed at $18-20 \mathrm{kD}$ following shortening exercise may represent myosin light chains in a phosphorylated and dephopsphorylated form. Following lengthening exercise, the spots assumed to represent myosin light chain 3 are less abundant and no double spots at the same molecular weight were detected.

If these spots do indeed represent myosin light chains and alterations in phosphorylation state take place following lengthening exercise, these alterations may have a functional role in contractile force development following shortening and lengthening exercise. However, immunoblotting with antibodies against the myosin light chain isoforms is required before conclusions can be drawn.

Lengthening exercise (L, SL and LL) induced 4 additional protein bands in the low molecular weight (15-20 kD) region, corresponding to histones, as concluded from their low pl. Histones are abundantly present in nuclei of virtually all eukaryotic cells. Therefore, increased expression of histones after lengthening exercise can be explained by the observation that after lengthening exercise, the affected muscles are infiltrated by mononuclear cells (see Fig 8.2).

In summary, the present study shows that $\alpha$-actinin is absent $4 \mathrm{~d}$ following lengthening exercise, in contrast to shortening exercise. Following lengthening exercise, vimentin was re-expressed together with desmin, indicating remodeling of the intermediate filament lattice. After a second lengthening exercise session, vimentin expression was less abundant. The data suggest that following lengthening exercise, remodeling and reorganization of the intermediate filament network takes place. Remodeling is accelerated in muscles previously subjected to lengthening exercise. Reorganization of the intermediate filament network may improve force transmission and consequently improve muscle function during a second lengthening exercise session. Whether altered expression of myosin light chains and its phosphorylation state contributes to improved muscle function remains to be elucidated. 


\section{References}

1. Amstrong, R. B., R. W. Oglivie, and J.A. Schwane. Eccentric exercise induced injury to skeletal muscle. I Appl Physiol 54: 80-93, 1983.

\section{Astrand, P. O., and K. Rodahl. Textboak of work} physilology, physiological bases of exercise. New York: Mc Graw Hill, 1988.

3. Balnave, C. D., and M. W. Thompson. Effect of training on eccentric exercise-induced muscle damage. I Appl Physiol 75: 1545-1551, 1993

4. Eifleter, R., M. Messerli, E. Wey, A. Puntschart, K. Jostandt $H . M$. Eppenberger, and $\rfloor$ C. Perriard. Fast myosin light chain expression in chicken muscles studied by in situ hybridization. I Histochem Cytochem 40: $15,47-57,1992$.

5. Clarkson, P. M., and I. Tremblay. Exercise-induced muscle damage, repair, and adaptations in humans. $J$ Appl Physiol 65: 1-6, 1988.

6. Debus, E., K. Weber, and M. Osborn. Monoclonall antibodies to desmin, the muscle-specific intermediate filament protein. EMBO J 2: 2305-12, 1983.

7. Ebbeling, C. B., and P. M. Clarkson. Exercise induced muscle damage and adaptation. Sports Med 7. 207-234, 1989.

8. Faulkner, J. A., D. A. Jones, and J. M. Round. Injury to skeletal muscles of mice by forced lengthening during contractions. QJ Exp Physio/ 74: 661-670, 1989.

9. Fridén, $d_{1}$, and IR. L. Lieber. Structural and mechanical basis of exercise-induced muscle injury. Med Sci Sports Exerc 2d: 521-30, 1992.

10. Hiolliwell, T. R., M. U. Jackson, J. Phoenix, P. Maclennan ${ }_{i}$ J. J. West, and R. H. Edwards. Immunohistochemicall and biochemical indicators of muscle damage in vitro: the stability of control muscle and the effects of dinitrophenol and calcium ionophore. Int J Exp Pathol 75: 329-43: 1994.

11. Hesselink, M. K. G. M. R. Drast, J. Komulainen, R. Uotila, H. A. Keizer, and H. Kuipers. Increased contraclile work during lengthening contractions after shortening and lengthening Iraining. Submitted.

12. Jones, D. A., and J. M. Round. Skeletai muscle in health and disease, a textbook of muscle physiology. Manchester: Manchester University press, 1993

13. Komulainen, J. R. Uotila, M. K. C. Hesselink, H. Kuipers, S. Koskinen, T. E. S. Takala, and V. Vihko. The damaging effect of forced lengthening contractions on skeletal muscle is followed by fibre hypertrophy. Biochemistry of Exercise Congress, 1997.

14. Kuruc, $K_{n, i}$ and W. W. Franke. Transient coexpression of desmin and cylokeratins 8 and 18 in developing myocardial cell of some vertebrates. Differentiation 38: 177-193, 1988,

15. Laeminli, U. K. Cleavage of structural proteins during the assembly of the head of bacteriophage $\mathrm{T}_{4}$ Nalure 227: $680-5,1970$.

16. Lazarides, E. Intermediate filaments as mechanical integrators of cellular space. Nature 283: 249-256, 1980 .

17. Lieber, R. L. Mecharnical factors underlying muscle damage. Second world congress of biomechanics, Amsterdam, p. 121, 1994.

18. Lieber, R. L., M. C. Schmitz, O. K. Mishra, and J. Friden. Contractile and cellular remodeling in rabbit skeletal muscle after cyclic eccentric contractions. $J$ Appl Physiol 77: 1926-34, 1994.

19. Lieber, R, L., L. E. Thomell, and J. Friden. Muscle cytoskeletal disruption oocurs within the first $15 \mathrm{~min}$ of cyclic eccentric contraction. J Appl Physiol 80: 278-84, 1996.

20. Lowe, D. A., G. L. Warren, C. P. Ingalls, D. B. Boorstein, and R. B. Armstrong. Muscle function and protein metabolism after initiation of eccentric contraction-induced injury. J Appl Physiol 79: 1260-1270, 1995.

21. Lowey, S., G. S. Waller, and K. M. Trybus. Skeletal muscle myosin light chains are essential for physiological speeds of shortening. Nature 365: 454456,1993

22. McCully, K. K., and J. A. Faulkner. Characteristics of lengthening contractions associated with injury to sikeletal muscle fibers. $J$ Appl Physiol 61: 293-299, 1986.

23. McCully, K. K. and J. A. Faulkner. Injuny to skeletal muscle fibers of mice following lengthening contractions. J Appl Physiol 59: 119-126, 1985.

24. Minner, D. J G. Weitzer, D. Tran, A. Bradley, and Y. Capetanaki. Disruption of muscle architecture and myocandial degeneration in mice lacking desmin. Cell Biol 134: 1255-1270, 1996.

25. O'Farrell, P. H. High resolution two-dimensional electrophoresis of proteins. J Biol Chem 250: 4007-21, 1975. 
26. Patel, T. J., and R. L. Leiber. Force transmission in skeletal muscle: from: actomyosin to external tendons. In: Exercise and Spont Sciences Reviews, edited by J. O. Holloszy. St. Louls: Williams \& Wilkins, p. 321-363, 1997.

27. Ramaekers, F. A. Huysmans, G. Schaart, 0 . Moesker, and P. Vooijs. Tissue distribution of keratin 7 as monitored by a monoclonal antibody. Exp Cell Res 170: $236-49$, 1987.

28. Sacco, P., and D. A. Jones. The protective effect of damaging eccentric exercise. Second world congres of biomechanics, Amsterdam, p. 123, 1994.

29. Schaart, G., C. Viebahn, H. Langmann, and F. C.S. Ramaekers. Desmin and titin expression in early postimplantation mouse embiryos. Development 107: 585-596, 1989,

30. Swiss prot. Swiss 20 page.

http:t/expasy.houge chicgi-bin/chad-search-de, 1997.

31. Tokuyasu, K. T., P. A. Maher, and S. J. Singer. Distributions of vimentin and desmin in developing chick myolubes in wivo. 1 Cell Biol 98: 1961-1972, 1984.

32. Towbin, $H_{1,}$ T. Staehelin, and J. Gordon. Electrophoretic transfer of profeins from polyacrylamide gels to nitrocelllulose sheets: procedure and some applications. Proc Natl Acad Sci US A A6: 4350-4, 1979.

33. Vandenboom, $R$, J. Xeni, N. M. Bestic, and M. E. Houstom. Increased force development rates of iatigued mouse skeletal muscle are graded to myosin light chain phosphate content. Am / Physiol, 1997.

34. Wong, T. S., and F. W. Booth. Protein metabolism in ral gastrocnemius muscle after stimulated chronic concentric exercise. J Appl Physiol 69: 1709-17, 1990.

35. Wong, T. S., and F. W. Booth. Protein metabolism in raf tibialis anterior muscle after stimulated chronic eccentric exercise. J Appl Physiol 69: 1718-24, 1990. 

General Discussion 


\section{Introductory remarks}

Skeletal muscle makes up approximately $40 \%$ of adult human body mass and permits interaction with the environment. During everyday movement, muscle contractions involve both lengthening and shortening components. Lengthening muscle contractions are associated with muscle damage $(1,16,21,23,32,33,40$, 41) and may as such affect muscle function and muscle metabolism. Information on the relationship between the duration of exercise (i.e., number of contractions) and the extent of muscie damage is fragmentary. Furthermore, little is known about the effect of muscle damage on energy metabolism and muscle function following damaging lengthening exercise. Finally, a rapid adaptation to damaging lengthening exercise is reported in the literature $(7,16,20,39,52,53,58)$. However, a mechanistic explanation is still lacking.

The general aim of the present thesis was to study skeletal muscle morphology, muscle function and muscle metabolism during and after single and repeated exercise sessions of shortening or lengthening muscle contractions. The studies outlined here were performed in vivo on rat tibialis anterior (TA) and extensor digitorum longus (EDL) muscles.

The TA-EDL complex was chosen for its accessibility. Moreover the common peroneal nerve, innervating the TA-EDL complex, is relatively easy to access. For histology and biochemical analysis only the midbelly region of the TA was prociessed. The TA muscle makes up $\sim 80 \%$ of the total mass of the exercised muscle group and TA muscle fibers extend $\sim 70 \%$ of the entire muscle length (35), therefore midbelly sections will represent most muscle fibers.

\section{Methodological considerations}

Two different experimental set-ups have been used in the experiments outlined in the present thesis. For the experiments discussed in chapter 3 and 4 a dynamometer was used permitting lengthening but not shortening exercise. Moreover, muscle function could only be recorded by assessment of isometric torque after an exercise session. To be able to monitor muscle function during movement, an Ashton-Miller-like (2) dynamometer was constructed.

The newly developed dynamometer used here differs from classical set-ups used by others $(2,11,12,21,32,35,62,63)$ in the way the rat is positioned in the dynamometer and in the way torque is measured and subsequently work is computed. The vast majority of the set-ups mentioned are based on a Cambridge ergometer system $(11,12,21,32,35,62,63)$. The Cambridge ergometer system has been used for small rodents (rats) as well as for rabbits. The disadvantage of using the Cambridge system in rat studies is the relatively high mass of the mobile components. Consequently, the moment of inertia is high and detection of small differences in work are biased by a relatively high inertial work. In the system diescribed by Ashton-Miller (2) this problem is overcome by the use of an other motor combined with light-weighed components and the use of a strain-gauged force transducer implemented in the shaft of the mobile unit. The implementation of the torque cell into the rotational axis causes a loss of stiffness of the axis. Loss of stiffness will bias torque recorded during acceleration and is therefore undesirable. Furthermore, except for the set-up used by Caiozzo et al. (12), all set-ups use a 
rigid fixation of the knee. Consequently, force may be transmitted by the tibia to the knee fixation unit and torque recorded by the dynamometer will be biased.

In the set-up presented here; the above mentioned problems are overcome by using a hinge fixation of the knee while torque is recorded without loss of stiffness by assessment of the voltage through the linear motor. Inertial torque was found to be very reproducible and was corrected for when calculating work. Work is calculated over the entire range of motion and work during a passive movement (comprising inertial work) is subtracted from work during contraction. Validation experiments, outlined in chapter 2, have shown that this new dynamometer permits valid and accurate measurement of muscle torque and computation of contractile work during lengthening and shortening contractions. In the present set-up " surgical intervention is not required for measuring work. Consequently, repetitive measurements within the same rat can be easily performed.

If muscle contractile performance is repeatedly monitored, care must be taken not to damage the nerve during electrical stimulation. Optimization of electrical stimulation during pilot-experiments, revealed that repeated measurement of contractile work within the same rats displayed similar results when recording work on successive days using percutaneous needle electrodes. This observation indicates that no damage to the peroneal nerve occurred. However, a shortcoming of the present way of electrical stimulation is that motor unit recruitment using high-voltage stimulation reverses the physiological motor unit recruitment pattern (27). Further refinement of electrical stimulation (8) is desired to mimic physiological stimulation of the muscle.

Muscle contractile function is most commonly studied by recording isometric torque. Since isometric torque is recorded at only one (optimal) muscle length, no information on muscle function during movements is obtained. In vivo muscle performance is more closely monitored by recording work during contraction on either side of the optimal muscle length than by recording isometric torque at a fixed muscle length. Therefore, we have chosen to use work, computed during the entire range of motion, as a functional parameter rather than using isometric torque.

\section{Declined torque versus declined work}

In chapter 3 we have shown that muscle torque recorded immediately following damaging exercise demonstrated a weak correlation with the extent of muscle damage. In the experiments outlined in chapter 3 , we showed that the relative decline in muscle torque is more pronounced after damaging than after nondamaging exercise. In contrast, we showed in chapter 7 that contractile work declined similarly during damaging and non-damaging exercise. The discrepancy in response of muscle torque and muscle work may have two origins.

Isometric torque was assessed post-exercise while work was recorded during the exercise session. Following non-damaging exercise, metabolic recovery may occur (chapter 4 and 5) and thus help to restore isometric torque. Energy metabolism is impaired following damaging exercise upto 24 and 48 hours after exercise (chapter 4 and 5 , respectively). Therefore, restoration of isometric torque, due to metabolic recovery during the time span between termination of damaging 
exercise and recording of muscle isometric torque, will not occur. Consequently, isometric torque recorded after non-damaging exercise exceeds isometric torque following damaging exercise. Work is recorded during exercise under damaging and non-damaging conditions, as a consequence, metabolic recovery will not affect the amount of work.

Isometric torque was assessed pre- and post-exercise at the same angle ankle $\left(90^{\circ}\right)$ and not at optimal muscle length (the length at which isometric peak torque occurs). Muscle damage has been hypothesized to originate from 'popping' sarcomeres while lengthening a muscle at the descending limb of the force length curve (42). Due to popping sarcomeres, sarcomere length heterogeneity increases following lengthening exercise (42). Consequently, optimal muscle length might be increased following damaging exercise and recording of isometric torque pre- and post-exercise at the same ankle angle may bias torque recorded after damaging exercise.

To shed more light upon the hypothesized changes in optimal muscle length, torque-angle relationship should be assessed before and after damaging lengthening exercise.

\section{Muscle glycogen resynthesis after damaging exercise}

Numerous reports exist on hampered muscle glycogen resynthesis following lengthening exercise $(17,18,22,29,45,46,48,61)$. To date, however, no mechanistic explanation has been given. In a series of experiments outlined in chapter 4 and 5 we tried to shed more light upon this unexplained phenomenon. In chapter 4 we have clearly shown that resynthesis of muscle glycogen is hampered following lengthening exercise. Resynthesis of muscle glycogen relies among others on the intracellular availability of glucose. Declined muscle glycogen resynthesis following lengthening exercise was shown to be associated with depressed activity of glycogen synthase for at least $48 \mathrm{~h}$ (chapter 4 and 5 ), which is in correspondence with observations of others (18). More recently, reports have shown that depressed activity of glycogen synthase is accompanied by declined expression of GLUT4 $(4,5)$, which in turn decreased contraction- and insulinmediated glucose uptake $(3,6,28)$. In chapter 6 we report on declined GLUT4 content immediately after lengthening exercise as well as 24 and $48 \mathrm{~h}$ later. In contrast, GLUT4 content returned to control values within $6 \mathrm{~h}$ after lengthening exercise. Although the reason for this bi-phasic response is at present not understood we will present a hypothetical sequence of events.

During lengthening exercise disruption of the cellular membrane occurs (13). Due to loss of membrane integrity GLUT4 may be released into the sarcoplasm and the extracellular space. Consequently, assessment of GLUT4 content in total crude muscle membranes may underestimate the actual GLUT4 content. Contraction-induced glucose transport has a half life time of $\sim 8 \mathrm{~min}(47)$, thus migration of GLUT4 to the sarcolemma will cease shortly after cessation of exercise and $6 \mathrm{~h}$ post-exercise the majority of GLUT4 will be in intracellular vesicles, preventing degradation of the GLUT4 protein. Twenty-four and $48 \mathrm{~h}$ after lengthening exercise, myofibrillar protein degradation (37) and cellular remodeling (34) peak and integrity of the GLUT4 containing vesicles may be lost (partly). 
Subsequently, GLUT4 may be exposed to substances degrading the GLUT4 protein. Studies monitoring the process of translocation in combination GLUT4 expression will shed light upon this hypothetical sequence of events. Immunohistochemical staining of GLUT4 at light microscopical level provides the means to study the presence/absence of GLUT4 in individual muscle cells. Serial cross-sections stained with periodic acid Schiff (PAS) for glycogen content and with hematoxilin-eosin for localization of muscle damage may help to study whether the decline in glycogen and GLUT4 are restricted to damaged muscle cells.

The observed decrease in insulin-and contraction-mediated glucose uptake after damaging lengthening exercise $(3,6,28)$ may originate from a defect in the translocation process. In healthy skeletal muscle, GLUT4 migrates from intracellular storage pools to the cellular membrane upon stimulation with insulin and/or after contractile activity $(24,25,49,51,56,59)$. Translocation of GLUT4 can be monitored by subcellular fractionation $(24,26,55)$, exofacial photolabeling $(19$, 38 ) and immunohistochemistry $(10,50,56,57,59)$. The latter technique would be helpful to study translocation of GLUT4 and glycogen resynthesis following lengthening contractions, if applied at light microscopical levell on serial sections.

\section{Remodeling of muscles subjected to lengthening exercise}

In chapter 7 we have shown that muscle damage is alleviated and muscle contractile performance declines less during a second lengthening exercise session if performed $15 \mathrm{~d}$ after the first exercise session. This rapid adaptation was restricted to lengthening exercise, shortening exercise did not possess a protective effect to damaging exercise. The rapid adaptation of skeletal muscle to damaging exercise is known as the repeated bout effect and is a frequently observed phenomenon $(7,14,15,39,43,44,53,54,58)$. However, information on the mechanisms underlying the repeated bout effect is scarce. In chapter 8 we have studied the expression of myofibrillar and cytoskeletal proteins following repeated lengthening exercise. Together with reports on adaptation to lengthening exercise $(9,31,34)$ data presented in chapter 8 indicate involvement of $\alpha$-actinin, desmin, vimentin and possibly myosin light chain isoforms in remodeling of muscles subjected to lengthening exercise. Involvement of the protein rulers in skeletal muscle (titin and nebulin) $(30,36,60)$ in remodeling of damaged skeletal muscle is likelly to occur but has not been established yet. Studying the expression of myofibrillar and cytoskeletal proteins on successive time points after lengthening exercise will extend the understanding of remodeling of skeletal muscle after damaging lengthening exercise and the reported rapid adaptation and should be the subject of further investigations. 


\section{Conclusions}

The main conclusions of the experiments outlined in the present thesis are:

- Muscle contractile work can be accurately assessed with minor surgical interventions using the dynamometer and the way of electrode positioning presented in this dissertation

- Repetitive assessment of muscle contractile work without inducing damage to the nerve can be performed using percutaneous needle electrodes

- Muscle damage increased with the number of lengthening contractions, while isometric or shortening contractions did not induce histological damage

- Muscle torque recorded immediately following damaging exercise demonstrated a weak correlation with the extent of histological muscle damage

- Using identical stimulation conditions, muscle glycogen declined similarly after shortening, lengthening and isometric exercise, indicating a similar metabolic requirement

- Following lengthening exercise a sustained (24 h) decrease in muscle highenergy phosphate content was observed

- Post-exercise restoration of muscle glycogen was hampered after damaging lengthening exercise which was accompanied by decreased activity of glycogen synthase and decreased expression of GLUT4

- The presence of GLUT4 and translocation upon stimulation with insulin or contractile activity can be qualitatively examined at light microscopical level by indirect immunofluorescence in serial cross-sections and may be helpful in studying the mechanism underlying the delayed glycogen resynthesis in damaged skeletal muscle

- Muscle damage is alleviated and muscle contractile work declines less rapidly during lengthening exercise in previously lengthened muscles. This is explained by remodeling of myofibrillar and cytoskeletal constituents following muscle damage which may be contribute to improved stress resistance and muscle performance of previously lengthened muscles 


\section{References}

1. Armsirong, R. B, R. W. Oglivie, and J. A. Schwane. Eccentric exercise induced injury to skeletal muscle. I Appl Physiol 54: 80-93, 1983.

2. Asthton-Miller, J. A., Y. He, V. A. Kadhiresan, D. A. McCubbrey, and J. A. Faulkner. An apparatus to measure in vivo biomechanical behavior of dorsi- and plantartlexors of mouse ankle. WAppl Physiol 72: 120511,1992

3. Asp, S., J. R. Daugaard, S. Kristiansen, B. kiens, and E. A. Fichter. Eccentric exercise decreases maximal insulin action in humans: musclle and systemic effects. J Physiol Land 494: 891-898, 1996.

4. Asp, S., J. R. Daugaard, and E. A. Richter. Eccentric exercise decreases glucose transporter GLUT4 protein in human skeletal muscie. J Physiol Lond 482: 705-712, 1995.

5. Asp, S. S. Kristiansen, and E. A. Richter. Eccentric muscle damage transiently decreases rat skeletal muscie GLUT-4 protein. J Appl Physiol 79: 1338-45, 1995.

6. Asp, S., and E. A. Fichter. Decreased insulin action on muscle glucose transport after eccentric contractions in rats. J App/ Physiol 81: 1924-1928, 1996.

7. Balnave, C. D., and M. W. Thompson. Effect of training on eccentric exercise-induced muscle darnage. J Appl Physiol 75: 1545-1551, 1993.

8. Baratta, R., M. llchie, S. K. Hwang, and M. Solomonov. Orderly stimulation of skeletal muscle motor units with tripolar nerve cuff electrode. IEEE Trans Biomed Eng 36: 836-843, 1989.

9. Booth, F. W., and D. B. Thomason. Molecular and cellular adaptation of muscle in response to exercise: perspectives of various models. Physiol Rev 71: 541-85, 1991.

10. Bornemann, A., T. Plousg, and H. Schmalbruch. Subcellular localization of GLUT4 in nonstimulated and insulin-stimulated rat soleus muscle. Diabeles 38 . 215-221, 1992

11. Caiozzo, V. J. M. J. Baker, and K. M. Baldwin. Madulation of myosin isoform expression by mechanical loading: role of stimulation frequency. $J$ Appl Physiol 82: 211-8, 1997.

12. Caiozzo, V. J., E. Ma, S. A. McCue, E. Smith, R. E. Herrick, and K. M. Baldwin. A new animal model for modulating myosin isoform expression by altered mechanical activity. J Appl Physiol 73: 1432-40, 1992.
13. Carter, G. T. N. Kikuchi, S. J. Horaselk, and S. A. Walsh. The use of fiuorescent dextrans as a marker of sarcolemmal injury. Histol Histopath 9: 443-447, 1994.

14. Clarkson, K. Nosaka, and B. Braun. Muscle function after exercise-induced muscle damage and rapid adaptation. Med Sci Sports Exerc 24: 512-520, 1992.

15. Clarkson, P. M., and C. Ebbeling, Investigation of serum kinase variability after muscle damaging exercise. Clin. Sci. 75: 257.261, 1988

16. Clarkson $\mathrm{P}, \mathrm{M}_{n}$ and I. Tremblay. Exercise-induced muscle damage, repair, and adaptations in humans. I Appl Physiol 65: 1-6, 1988.

17. Castill, D. L., D. D. Pascoe, W. J. Fink, R. A. Robergs, S. I. Barr, and D. Pearson. Impaired muscle glycogen resynthesis after eccentric exercise. J Appl Physiol 69: $46-50,1990$.

18. Doyle, J. A., W. M. Sherman, and F. L. Strauss. Effects of eccentric and concentric exercise on muscle glycogen replenishment. I Appl Physiol 74: 1848-1855. 1998.

19. Dudek, A. W., G. L. Dohm, G. D. Holman, S. W. Cushman, and C.M. Wilson. Glucose transporter localization in rat skelletal muscle. Auto-radiographic stduy using ATB-(2-3H)BMPA photolabel. FEBS Lett 39: 205-208, 1994 .

20. Ebbeling, C. B., and P. M. Clarkson. Exercise induced muscle damage and adaptation. Sports Med" 7: 207-234, 1989

21. Faukner, J. A., D. A. Jones, and J. M. Hound, Injury lo skeletal muscles af mice by lorced lenghthening during contraclions، Q J Exp Physiol 74: 66 1-670, 1999.

22. Ferry, A. I. Amiridis, and M. Rieu. Glycogen repletion and synthesis in the ral after downhill running Eur J Appl Physiol 64: 32-35, 1992.

23. Fridén, J. Exercise-induced muscle soreness: a qualitative and quanitative study of humarn muscie imorphology and lunction. Umeá Universily Medical Dissertations New Series, 1983.

24. Goodyear, L. J. M. F. Hirshman, and E. S. Horton. Exercise-induced translocation of skeletal muscle glucose transporters. Am J Physiol 0054, 1991.

25. Goodyear, L. J., M. F. Hirshman, F. Napoli, J. Calles J. F. Markuns, O. Ljungquist, and E. S. Honton. Glucose ingestion causes GLUT4 translocation in human skeletal muscle. Diabetes 45: 1051-68, 1996 . 
26. Goodyear, L. d. P. A. King, M. F. Hirstiman, C. M. Thompson, E. D. Horton, and E. S. Hortion. Contractile activity increases plasma membrane glucose transporters in abserice of irsulin. Am J Physiol 0054 . 1900.

27. Henneman, E., H. P. Clantanm, U. D. Gillies, and R. D. Skinner. Rank order of motomeuons within a pool, law of combination. I Neurol Sci 37: 1338-1349, 1974.

28. Kristiansen, S., S. Asp, and E. A. Richer. Decreased muscle glut 4 and contraction induced glucose transporl after eccentric contractions. American Joumal Of Physiology Regulatory Integrative And Comparative Physiology. Aug 40: R477-R482, 1996.

29. Kuipers, H. H. A. Keizer, F. T. J. H. Verstappen, and D. L. Costill. Influence of a prostaglandin-inhibiting drug on muscle soreness after eccentric work. In $J$ Sports Med 6: 336-339," 1985.

30. Labeit, $S$, and B. Kolmerer, The complete primary structure of human nebulin and its correclation to muscle structure. J Mol Biol 248: 308 $315_{4} 1995$.

31. Lapier, T. K., H. W. Burton, R. Almon, and F. Cerny. Alterations in intramuscular connective tissue after limb casting effect contraction-induced muscle injury. J Appl Physiol 78: 1065-1069, 1995.

32. Lexell, J., J. Jarvis, D. Downham, and S. Salmons, Quantitative morphology of stimulation-induced damage in rabbit fast-twitch skeletal muscle. Celf Tissue Res 269: 195-204, 1991.

33. Lieber, R. L. Mechanical factors underlying muscle damage. Second world congress of biomechanics. Amsterdam, 1994, p. 121.

34. Lieber, R. L., M. C. Schmilz, D. K. Mishra, and J. Friden. Contractile and cellular remodeling in rabbit skelelal muscle after cyclic eccentric contractions. $J$ Appl Physiol 77: 1926-34, 1994.

35. Lieber, R. L., T. M. Woodburn, and J. Friden. Muscle damage induced by eccentric contractions of 25\% sirain. J Appl Physiol 70: 2498-507, 1991.

36. Lin, Z, M. H. Lu, T. Schultheiss, J. Choi, S. Holtzer, C. DiLullo, D. A. Fischman, and H. Holtzer. Sequential appearance of muscle-specific proteins in myoblasts as a function of time after cell division: evidence for a conserved myoblast differentiation program in skeletal muscle. Cell Motil Cytoskeieton 29: 1-19, 1994.

37. Lowe, D. A G. G. L. Warren, C. P. Ingalls, D. B. Boorstein, and R. B. Armstrong. Muscle function and protein metabolism after initiation of eccentric contraction-induced injury. J Appl Physiol 79: 1260-1270, 195

38. Lund, S., G. D. Holman, O. Schmitz, and O. Pedersen. GLUT4 content in the plasma membrane of ral skeletal muscle: comparative studies of the subcellular fractionation method and the exofacial photolabelling lechnique using ATB-BMPA. FEBS Lett 312-318, 1903 .

39. Mair, J., M. Mayr, E. Müller, A. Koller, C. Haild, E. Artner-Dworzak, C. Calzolari, C. Larue, and B. Puschendorf. Riapid adaptation to eccentric exerciseinduced muscle damage. Int $J$ Sports Med 16: 352-356, 1094.

40. McCully, K. KK, and J. A. Faulkner. Characteristics of lengthening contractions associated with injury to skeletal muscle fibers. J'Appl Physiol 61: 293-299, 1986.

41. McCully, K. K., and J. A. Faulkner. injury to skeletal muscle fibers of mice following lengthening contractions. I Appl Physiol 59: 119-126, 1985.

42. Morgan, D. L. New insights into the behaviour of muscle during active lengthening. Biophys $\sqrt{ }$ 57: 209221,1990

43. Newham, D. J., D. A. Jones, and P. M. Clarkson. Repeated high-force eccentric exercise: effects on muscle pain and damage. J Appl Physiol 63: 1381-6, 1987 .

44. Nosaka, K., and P. M. Clarkson. Muscle damage following repeated bouts of high force eccentric exercise. Med Sci Sponts Exerc 27: 1263-1269, 1995.

45. OReilly, K. P., M. J. Warhol, A. A. Fielding, W. R. Frontera, C. N. Meredith, and W. J. Evans. Eccentric exencise-induced muscle damage impairs muscle glycogen repletion. J App Physial 63: 252-256, 1987.

46. Pascoe, D. D., D. L. Costill, W. J. Fink, R. A. Fobergs, and J. J. Zachwieja. Glycogen resynthesis in skeletal muscle following resistive exercise. Med Sci Sports Exerc 25: 349-354, 1993.

47. Ploug, T., H. Galbo, J. Vinten, M. Jorgensen, and $E$. A. Richter. Kinetics of glucose transport in rat muscle: effects of insulin and contractions. Am J Physiol Endocrinal Metab 16: E12-E20, 1987.

48. Richter, E. A., L. P. Garetto, M. N. Goodman, and N. B. Ruderman. Muscle glucose metabolism following exercise in the rat. J Clin inves: 69: 785-793, 1982. 
49. Rodnick, K. J, R. C. Piper, J. W. Slot, and D. E. James. Interaction of insulin and exercise on glucose transport in muscle. Diabetes care 15: 1679-1689, 1992.

50. Rodnick, K. J. J.W. Slot, D. R. Studelska, D. E. Hampeter, L. J. Robinson, H. J. Geuze, and D. E. James. Immunocytochemical and biochemical studies of GLUT4 in rat skeletal muscle. The jounal of biological chemistry 267: 6278-6285, 1992.

51. Roy $D_{1}$, and $A$. Mareite. Exercise induces the translocation of GLUT4 to transwerse tubules from an intracellular pool in rat skeletal muscle. Biochem Biophys Res Commun 223: 147-52, 1996.

52. Sacco, $P_{\text {. }}$ and D. A. Jones. The protective effeet of damaging eccentric exercise. Second world congres of biomechanics, Amsterdam, i994, p. 123.

53. Sacco, $P$, and D. A. Jones. The protective effect of damaging eccentric exercise against repeated bouts of exercise in the mouse tibialis anterior muscle. Experimentai physiology 77: 757-760, 1992.

54. Schwane, J. A., and R. B. Armstrong. Effect of training on skeletal muscle injury from downhill running in rats. J Appl Physiol 55: 969-75, 1983.

55. Sherman " L. A A. M. F. Hirshman, M. Cormont, M. B. $Y . L e$, and L. J. Goodyear. Ditferential effects of insulin and exercise on Rab4 distribution in rat skeletal muscle. Endocrinology 137: 266-73, 1996.

56. Slot, J. W., H. Geuze, S. Gigengack, G. E. Lienhard, and D. E. James. Immuno-localization of the insulin regulatable glucose transporter in brown adipose lissue of the rat. I Cell Biol 113: 123-135, 1991.

57. Slot, J. W., H. J. Geuze, S. Gigengack, D. E. James, and G. E. Lienhard. Translocation of the glucose transporter GLUTA in cardiac myocyles of the rat. Proc Natt Acad Sci USA 88: 7815-7819, 1991

58. Smith, L. L., M. G. Fulmer, D. Holbert, M. R. McCammon, J. A. Houmard, D. D. Frazer, E. Nsien, and R. G. Israel. The impact of a repeated bout of eccentric exercise on muscular strength, muscle soreness and creatine kinase. Br J Sp Med 28: 267-271, 1994.

59. Smith, R. M. M. J. Charron, N. Shah, H. F. Lodish, and L Jarett. Immunoelectron microscopic demonstration of insulinestimulated translocation of glucose iransporters to the plasma membrane of isolated rat adipocytes and masking of the carboxylterminal epitope of intracellular GLUT4. Proc Nall Acad Sci U S.A 88: 6893-7, 1991.
60. Trinick, J. Titin and nebulin: protein rulers in muscle? Trends Biachem Sci 19: 405-409, 1994.

61. wan der Meulen, J. H, H. Kuipers, F. R. M. Stassen, H. A. Keizer, and G. J. van der Wusse. High energy phosphates and related compounds, glycogen levels and histology in the rat tibialis anterior muscle after forced lengthening and isometric exercise. Pflugers Arch 420: 354-358, 1992.

62. Wong, T. S., and F. W. Booth. Protein metabolism in rat gastrocnemius muscle after stimullated chronic concentric exercise. J Appl Physiol 69: 1709-17, 1990.

63. Wong, T. S., and F. W. Booth. Protein metabolism in rat tibialis anterior muscle after stimulated chronic eccentric exercise. J Appl Physial 69: 1718-24, 1990. 



\section{Summary}




\section{Summary}

Skeletal muscle comprises approximately $40 \%$ of adult human body mass. Based on their mass, skeletal muscle is de largest organ in the human body. Skeletal muscle is made up of different proteins, part of which are able to generate force due to their unique structure and interaction (the so-called contractile proteins). Part of the remaining proteins are designed to transmit force generated by the contractile proteins to the skeleton (the so-called cytoskeletal proteins). Due to their highly organized structure, skeletal muscle is capable to initiate movements as well as to stop locomotion.

Every movement initiated by muscle contraction requires conversion of chemical energy into mechanical energy. In skeletal muscle, breakdown of carbohydrates and fatty acids are the main pathways for generating chemical energy. During physical exercise the amount of chemical energy required to fulfill the increased demand for mechanical energy increases dramatically. Regular physical exercise induces adaptation of the exercised muscles to the increased workload. These adaptations involve energy metabolism as well as adaptations at the level of myofibrillar and cytoskeletal proteins. Adaptation of the exercised muscle depends among others on the type of contraction that is employed.

Force development in skeletal may occur without a change in muscle length, e.g., while holding a load at a fixed elbow angle (isometric contraction). When a load is lifted and the muscle shortens, this is called a concentric or shortening contraction. Skeletal muscle is also capable to generate force while lengthening,e.g., while lowering a weight. This type of contraction is called an eccentric or lengthening contraction. In the present thesis we studied the effect of type of contraction on muscle structure and muscle function during, as well as after, shortening and lengthening contractions.

Chapter 1 is an introduction into muscle structure, force generation and transmission in skeletal muscle, as well as into generation of chemical energy. This chapter ends with defining the aims of the present thesis. To be able to assess muscle function in anaesthetized rats we designed a new experimental set-up. This set-up permits assessment of work generated by the muscle in an accurate and reproducible manner with minimal surgical intervention. The experiments outlined in chapter 3, confirm the well-known phenomenon that lengthening contractions induce damage to the exercised muscles whereas isometric contractions do not. The extent of muscle damage is exponentially related to the number of lengthening contractions. Furthermore, we observed that maximal muscle torque declined more following lengthening exercise than following isometric exercise. A weak correlation $(R=0.593)$ was observed between the number of lengthening contractions and the post-exercise force deficit between isometrically and eccentrically axercised muscles. Chapter 4 describes the effect of eccentric and isometric contractions on the metabolic status of the muscle immediately and 24 hours after exercise. Directly after both types of contraction, we observed a decline in total adenine nucleotide content. However, the decline after lengthening exercise was detectable already after 60 contraction and was more pronounced than following isometric contractions. Adenine nucleotide content remained depressed for at least 24 hours post-exercise. In concert with the decline in total 
adenine nucleotide content, we observed a rise in the concentration of IMP. Twenty-four hours after lengthening exercise, MMP returned to normal levels while the content of total adenine nucleotides was still depressed. This may indicate a loss of adenine nucleotides from the muscle after lengthening exercise. In the experiments described in chapter 4 we also observed that 24 hours after lengthening exercise, in contrast to after isometric exercise, muscle glycogen stores were still depressed. The deplorable metabolic status 24 hours after lengthening exercise most likely affects the force deficit observed in chapter 3 . For a better understanding of the profile of glycogen resynthesis after lengthening exercise, the experiments outlined in chapter 5 were performed. In these experiments we monitored muscle glycogen content as well as the activity of glycogen regulatory enzymes, together with muscle GLUT4 content, immediately, 6,24 and 48 hours after shortening and lengthening exercise. It was observed that 6 hours after both types of exercise muscle glycogen did not differ from control values. However, the rate of glycogen resynthesis was lower after lengthening exercise than after shortening exercise. Remarkably, 24 and 48 hours after lengthening exercise, muscle glycogen dropped far below control values while after shortening contractions muscle glycogen content stabilized at control values. This bi-phasic response of muscle glycogen to lengthening exercise perfectly matches the response of muscle GLUT4 content to lengthening exercise. Since the activity of glycogen synthase remained depressed for the entire 48 hour postexercise period, we postulate that muscle GLUT4 content is of greater importance for glycogen resynthesis after lengthening exercise than is activity of glycogen synthase. Transport of glucose across the cellular membrane of skeletal muscle largely relies on the presence of GLUT4 in the membrane. Under resting conditions these glucose transporting proteins are found in intracellular vesicles. Upon stimulation with insulin, and/or after contractile activity, these transporters migrate to the cellular membrane (translocation) to enhance glucose uptake several fold. In chapter 6 we qualtiatively monitored the process of translocation at light microscopical level by using specific antibodies and fluorescence microscopy. A weak signal in vicinity of the cellular membrane was observed under resting conditions which, however, intensified upon stimulation with insulin and after contractile activity. This observation indicates that translocation of GLUT4 is detectable at light microscopical level. The technique used to monitor the translocation process can be easily combined with other staining techniques to study, for example, muscle glycogen depletion. Chapter 7 shows that muscles subjected to lengthening exercise twice, within 2 weeks, are able to generate more work and are less vulnerable to damage than muscles subjected to lengthening exercise preceded by shortening exercise 2 weeks before. This observation indicates a rapid adaptation to lengthening exercise. In chapter 8 we studied the role of myofibrillar and cytoskeletal proteins the process of adaptation to lengthening exercise. Using one- and two-dimensional gel electrophoresis we studied the profile of protein expression after shortening and lengthening exercise. A single shortening exercise session did not induce alterations in the protein expression profile of the exercised muscles, compared to non-exercised muscles. Four days after lengthening exercise, however, we observed a co-expression of the 
cytoskeletal proteins desmin and vimentin, as is commonly observed during early myogenesis. After a second lengthening exercise session these alterations are less prominent. Lengthening exercise also induced a decrease in expression of $\alpha$ actinin and myosin light chain 1,2, and 3. The reported changes in the protein expression profile indicate that both myofibrillar and cytoskeletal proteins are involved in remodeling of skeletal muscle after a single lengthening exercise session. No such alterations were detectable after a single shortening exercise session. 


\section{Samenvatting}




\section{Samenvalting}

Het lichaamsgewicht van een volwassen mens wordt voor ongeveer $40 \%$ bepaald door het gewicht van de skeletspieren. Hiermee wormen de skeletspieren, gerekend naar hun massa, het grootste orgaansysteem in het menselijk lichaam. Skeletspieren zijn opgebouwd uit verschillende soorten eiwitten waarvan een deel door hun unieke opbouw en interactie in staat zijn kracht te genereren (de zogenaamde contractiele eiwitten) en een ander deel (o.a. cytoskelletaire eiwitten) met name geschikt is om kracht, die onstaat bij het activeren van een spier, te transporteren naar het skelet. Door hun unieke opbouw zijn skeletspieren in staat om bewegingen te initiëren en reeds geïnitieerde bewegingen weer af te remmen.

Voor iedere beweging die door spieren tot stand gebracht wordt is de omzetting van chemische naar mechanische energie nodig. De chemische energie die in de spier gegenereerd wordt komt met name voort uit de afbraak van suikers (koolhydraten) en vetzuren. Tijdens lichamelijke inspanning neemt de hoeveelheid chemische energie, benodigd om aan de verhoogde vraag naar mechanische energie te voldoen, enorm toe. Wanneer men met enige regelmaat lichamelijk actief is zullen de actieve spieren zich aanpassen aan hun nieuwe activiteiten patroon. Dergelijke aanpassingen vinden zowel plaats op metabool energetisch niveau (ten behoeve van de chemische energie voorziening) als op het niveau van de contractiele en cytoskeletaire eiwitten (ten behoeve van de mechanische energie leverantie). De wijze waarop, alsmede de tijdspanne waarin, de spier zich aanpast aan een nieuw activiteiten patroon hangt onder andere samen met de omstandigheid waaronder de spier kracht levert.

Een spier kan kracht leveren terwijl zijn lengte niet verandert, zoals bij dragen van een last met een constante gewrichtshoek. Dit heet een isometrische contractie. Wanneer de spier verkort bijvoorbeeld bij het optillen van een last spreken we van een concentrische contractie. Echter een spier kan ook kracht leveren terwijl hij langer wordt, bijvoorbeeld bij het langzaam laten zakken van een last. In dat laatste geval spreken we van een excentrische contractie. In het onderzoek dat beschreven is in dit proefschrift, hebben we het effect bestudeerd van verschillende soorten spiercontracties op de structuur van de spier, de wijze waarop de spier mechanisch functioneert tijdens en na concentrische en excentrische contracties en de veranderingen in de energie stofwisseling die optreden bij concentrische en excentrische spiercontracties.

Hoofdstuk 1 is een inleidend hoofdstuk waarin achtereenvolgens beschreven wordt hoe de skeletspier is opgebouwd, hoe in skeletspier kracht geproduceerd en doorgegeven wordt, en hoe in skeletspieren chemische energie wordt gegenereerd. Deze inleiding mondt uit in de formulering van de doelstellingen van ons onderzoek. De experimenten beschreven in dit proefschrift zijn uitgevoerd bij verdoofde ratten. Teneinde bij verdoofde ratten de spierfunctie te kunnen meten hebben we een experimentele opstelling ontwikkeld die ons in staat stelt om, met een minimale chirurgische ingreep, een spiergroep te activeren via electrostimulatie en op betrouwbare en herhaalbare wijze de geleverde arbeid te meten. De karakterisering en validering van de gebruikte meetopstelling en de gevolgde procedures zijn beschreven in hoofdstuk 2. De experimenten beschreven in hoofdstuk 3 bevestigen het reeds bekende fenomeen dat bij 
excentrische contracties spierschade optreedt die zich uit in o.a. ontstekingsreacties 24 uur na inspanning, dit in tegenstelling tot isometrische contracties. Uit de experimenten beschreven in dit hoofdstuk blijkt tevens dat de hoeveelheid schade exponentieel toeneemt met het aantal contracties. Na excentrische contracties blikt de maximale kracht die de spier kan generen sterker af te nemen dan na isometrische contracties. De grotere krachtsafname die we na excentrische contracties meten ten opzichte van de krachtsafname na eenzelfde hoeveelheid isometriche contracties, correleert zwak $(R=0.593)$ met de hoeveelheid opgetreden schade na excentrische inspanning. In hoofdstuk 4 is het effect van excentrische en isometrische contracties op de energie status van de spier direct na inspanning en 24 uur later. Direct na inspanning is er sprake van een daling in de totale hoeveellheid adenine nucleotiden, de directe bron voor chemische energie in de spier. Deze daling zien we zowel na isometrische als na excentrische contracties. Echter de daling na excentrische contracties die reeds na 60 contracties detecteerbaar is, is meer uitgesproken dan na isometrische contracties en houdt minstens gedurende de eerste 24 uur na excentrische arbeid stand. Met de daling in adenine nucleotiden wordt direct na inspanning een overeenkomstige stijging van IMP gerapporteerd, een afbraak product van één van de adenine nucleotiden. Wanneer we echter 24 uur na excentrische arbeid kijken valt op dat de concentratie IMP weer gedaald is tot een normaal niveau, terwijl cle totale hoeveelheid adenine nucleotiden nog verlaagd is. Dit duidt er op dat een deel van de adenine nucleotiden de spier die excentrisch belast is geweest verlaten heeft, mogelijkerwijs ingegeven door het feit dat de excentrische belaste spieren beschadigd zijn. Tevens is uit de experimenten, beschreven in hoofdstuk 4. gebleken dat 24 uur na excentrische arbeid de voorraad suikers in de spieren (spierglycogeen), nodig om energierijke verbindingen tot stand te brengen, nog steeds verlaagid is. Dit in tegenstelling tot 24 uur na isometrische arbeid, wanneer de concentratie spierglycogeen niet meer significant verschilt van controle waarden. De slechte metabool energetische toestand, waarin excentrisch belaste spieren zich tot 24 uur na inspanning bevinden, wordt verondersteld bij te dragen aan dalling in kracht zoals die in hoofdsuk 3 beschreven is. Teneinde een beter beeld te krijgen van het profiel van de nieuwvorming van spierglycogeen ma excentrische en concentrische inspanning hebben we de experimenten, zoals beschreven in hoofdstuk 5, uitgevoerd. Bij deze experimenten hebben we direct na inspanning, alsmede 6,24 en 48 uur later, de hoeveelheid spierglycogeen gemeten. Tegelijkertijd is de activiteit van de belangrijkste enzymen die de afbraak en opbouw van spierglycogeen reguleren bepaald en is de hoeveelheid GLUT4 gemeten (kwantitatief gezien het belangrijkste transport eiwit van suikers over de wand van de skeletspiercel). Uit deze studie is gebleken dat de mate van nieuwvorming van spierglycogeen 6 uur na excentrische arbeid lager is dan na concentrische arbeid, maar in beide gevallen niet meer significant lager is dan controle waarden, hetgeen direct na inspanning wel het geval is. Echter, 24 en 48 uur na inspanning is de hoeveelheid spierglycogeen in de excentrisch belastte spieren weer gedaald tot ver onder de uitgangs niveaus. Dit in tegenstelling tot na concentrische contracties, wanneer spierglycogeen binnen 6 uur na inspanning terug is op het uitgangsniveau en gedurende 48 uur stabiel blijft. De respons in 
glycogeen nieuwvorming na excentrische inspanning vertoont hetzelfde opmerkelijke bi-fasische verloop als de concentratie GLUT4. Omdat gedurende de gehele periode na inspanning de activiteit van het enzym dat zorgl voor de glycogeen nieuwvorming (glycogeen synthase) verlaagd is, lijkt het erop dat na excentrische arbeid de concentratie GLUT4 in de skeletspier van groter belang is bij glycogeen nieuwvorming dan de activiteit van glycogeen synthase. Opname van suikers door de skeletspier wordt in belangrijke mate bepaald door de aanwezigheid van GLUT4 in de wand van de skeletspiercel. Deze suiker transporterende eiwitten kunnen zich onder invloed van het hormoon insuline en/of spiercontractie verplaatsen naar de wand van de skeletspiercel (translocatie), alwaar ze het transport van suikers over de celwand vele malen kunnen verhogen. In hoofdstuk 6 is middels specifieke antilichamen en fluorescentie microscopie getracht deze suiker transporterende elwitten en het translocatie proces in beeld te brengen. Deze studie toont aan dat er sprake is van een zwak signaal in de celwand van de rustende skeletspier dat echter intenser wordt onder invlloed van insuline of spieractiviteit. Dit duidt erop dat translocatie van GLUT4 op licht microscopisch niveau aantoonbaar is. De techniek die gebruikt is om het translocatie proces in beeld te brengen kan ook gebruikt worden om te bestuderen of de verminderde glycogeen nieuwvorming en GLUT4 hoeveelheid (zoals gerapporteerd in hoofdstuk 5) zich beperkt tot spiercellen die door excentrische arbeid daadwerkelijk beschadigd zijn of dat het probleem zich ook in onbeschadigde spiercellen voordoet. In hoofdstuk 7 hebben we aangetoond dat spieren die binnen 2 weken voor een tweede keer onderworpen worden aan een serie excentrische contracties in staat zijn meer arbeid te leveren en minder beschadligd worden dan spieren die 2 weken eerder concentrisch belast zijn. Het vermogen meer arbeid te kunnen leveren tijdens een tweede sessie excentrische contracties, alsmede de verminderde schade na de tweede excentrische inspannings sessie, duidt op een snelle adaptatie van de skelletspier aan excentrische inspanning. In hoofdstuk 8 is bekeken of er contractiele en/of cytoskeletaire eiwitten zijn die een rol zouden kunnen spelen bij deze snelle adaptatie. Middels één en twee-dimensionale gel electrophorese en immumoblotting is het patroon van eiwit expressie na concentrische en excentrische inspanning bestudeerd. Na concentrische inspanning zien we geen verschillen in de expressie van deze eiwitten, vergeleken met een controle spier. Vier dagen na de eerste serie excentrische contracties valt op dat de cytoskeletailre eiwitten desmine en vimentine samen tot expressie komen, een situatie die normaal alleen aangetroffen wordt tijdens embryologische ontwikkeling van spier. Na een tweede serie excentrische contracties zijn deze veranderingen minder uitgesproken. De expressie van $\alpha$-actinine en de lichte myosine ketens is tevens verminderd na excentrische arbeid. Gezamelijk duiden de veranderingen in het eiwit expressie patroon erop dat er na excentrische inspanning aanpassingen plaatsvinden in de expressie van zowel de contractiele als de cytoskeletaire eiwitten. Dergelijk veranderingen zijn niet detecteerbaar na ến serie concentrische contracties. 


\section{Dankwoord}

Op een regenachtige zaterdagochtend in het begin van 1998 sta ik, zoals wel vaker de afgelopen periode, in dubio. lets in me zegt dat het misschien verstandig is deze wat druilerige ochtend te besteden aan het schrijven van een woord van dank aan diegenen die mij gedurende mijn promotie op velerlei wijze gesteund hebben. Aan de andere kant heb ik zin om een lekker rondje te gaan fietsen. Na een kort overleg met het thuisfront sta ik buiten in de motregen, klaar voor 'een rondje Aubel'. ljs, weder en vorm dienende duurt zo'n rondje vanuit Maastricht een kleine 2 uur, misschien nét voldoende tijd om te bedenken wie ik vooral niet moet vergeten te bedanken. Nog voor ik Oost-Maarland gepasseerd ben kom ik tot de conclusie dat de meeste dankwoorden in proefschriften in meer of mindere mate allemaal clichématig maar zonder twijfel welgemeend zijn. Ter geruststelling; het dankwoord in dit proefschrift zal wat dat betreft niet veel afwijken van de norm.

De eerste die ik in dit dankwoord wil noemen is Martine. Jou bijdrage aan de totstandkoming van dit proefschrift verschilt in allerlei opzicht van die van anderen die in dit dankwoord genoemd zullen worden. De harmomieuze wijze waarop we al zo lang bij elkaar zijn heeft geleid tot een relatie die het toestaat bij tijd en wijle wat meer te werken dan goed is voor een relatie. Ik ben je erg dankbaar voor de wijze waarop je me stimuleert op momenten dat ik dat nodig heb en zeker niet minder voor het feit dat je me ook afremt op tijden dat dat nodig is. Ik hoop samen met jou en Marijn nog vele gelukkige jaren te beleven.

Uiteraard ben ik veel dank verschuldigd aan mijn directe begeleidings team bestaande uit promotor Prof. dr. H. Kuipers en beide co-promotores Dr. H.A. Keizer en Dr. ir. M.R. Drost. Harm bedankt voor het vertrouwen dat je van het begin af aan in me gehad hebt en hebt gehouden, ook wanneer ik met ideeën kwam waarvan je (soms terecht) de haalbaarheid ter discussie stelde. Hans, jou creativiteit en tomeloze enthousiasme voor wetenschappelijk onderzoek heb ik als erg stimulerend en motiverend ervaren. Je hebt mij overtuigd van het feit dat de moleculaire cel biologie een vaste waarde wordt (is) in fysiologisch onderzoek. Maarten, je hebt me direct en veelvuldig begeleid bij mijn eerste wankele stappen op het gebied van de skeletspier biomechanica. Het feit dat de afgelopen paar jaar het meten van spierfunctie middels een plastic bekertje met suikerklontjes zich heeft ontwikkeld tot het meten met 4 verschillende high-tech meetopstellingen komt voor een groot deel op jou conto. Het skeletspierfunctie onderzoek binnen onze vakgroep heeft, mede door jou inbreng, een flinke impuls gekregen. Je inmiddels alom gebruikte statement 'dubbelzijdig tape is beter dan alles' is meermalen waar gebleken, dit in tegenstelling tot je rotsvaste overtuiging dat taurine géen aminozuur is. De leden van de beoordelingscommisie, Prof. dr. ir. W. Saris, Prof. dr. F. Ramaekers, Dr. H. Savelberg, Prof. dr. G. van der Vusse en Dr. A. Wagenmakers will ik bedanken voor het kritisch doorlezen van dit proefschrift. 
Speciale dank gaat uit naar Ger en Frans voor hun inhoudelijke bijdragen aan respectievelijk hoofdstuk 4 en hoofdstuk 8 . Ton, ook jou wil ik bedanken woor de getoonde intresse en de stimulerende en vaak verfrissende discussies. Voor de zo broodnodige begeleiding bij mijn eerste dierexperimenten will ik Peter Geurten en Eric van Breda bedanken, beiden hebben mij ook wegwijs gemaakt in de wereld van de electronenmicroscopie en immunocytochemie waar dik en dun en groot en klein relatieve begrippen bleken te zijn. Peter, ik vind het jammer dat ik niet langer met je gewerkt heb, maar ik heb ontzettend veel van je geleerd. Eric onze wegen zullen in de toekomst nog wel kruizen en ik verwacht dat we dan nog wel het én en ander voor ekkaar kunnen betekenen. Veel van de biochemische analyses gedurende mijn promotie zijn, op vaak onnavolgbare wijze, door Gerrit van Kranenburg verzorgd. Werk dat niet zelden ondankbaar lijkt maar van onschatbare waarde is. Gerrit als je ooit op een willekeurige zondag door een trimmer uit de wind moet worden gezet dan bel je maar. Yvonne, door jou doorzettingsvermogen heb je samen met Dr. de Blotgors de immunoblotting voor GLUT4 opgezet, ik heb dlaar dankbaar gebruik van gemaakt. Paul Bomans, Peter Frederik en Rein van Gool van electronenmicroscopie wil ik danken voor hun altijd hartelijke wellkom en bruikbare adviezen als we weer eens een poging waagden om GLUT4 in beeld te brengen. De medewerkers van de centrale technische dienst van de technische universiteit Eindhoven en in het bijzonder Erwin Dekkers en Rinus Janssen wil ik bedanken voor de samenwerking die uitgemond heeft in onder andere de rattendynamometer die voor het merendeel van de experimenten beschreven in dit proefschrift gebruikt is. Henny van Straaten wil ik hartelijk bedanken voor zijn constructieve bijdrage aan hoofdstuk 3 , ik heb je coöperatieve instelling erg gewaardeerd en hoop daar ook in de toekomst nog af en toe gebruik van te mogen maken. Een bijzonder woord van dank gaat ook uit naar Hellma Kuijpers die telkens bereid was om snel weer een 'eiwit gelletje te draaien' dat, voor mij verbluffend snel, vaak bijzonder leuke resultaten heeft opgeleverd. Zonder jou medewerking (en die van Frans) had dit proefschrift een interessant hoofdstuk moeten missen.

Vanaf Oast-Maarland staat de wind stevig op kop maar inmiddels heb ik het klimmetje naar Bombaye (B) bedwongen en ben ik er met mijn gedachten niet helemaal meer bij. Ik overweeg de mensen te bedanken bij wie ik de afgelopen jaren wel eens het één en ander 'gebietst' heb, hetzij kennis, hetzij chemicaliën, of spulletjes die anderszins van pas komen op het lab. Omdat ik voorzie dat deze lijst mensen zo lang zal zijn dat ik die niet bij elkaar verzonnen heb voor ik weer in Maastricht ben besluit ik alleen de meest 'geplukten' te bedanken.

Paul van Dijk, Johan Hekking, Joan Senden, Paul van Schoffelen, Yvonne de Jong (op de dagen dat ze bij fysiologie werkt), Michaël Vork, Paul Bomans en Rein van Gool, allen bedankt voor het min of meer vrijwillig af staan van kennis en/of middelen. Vanzelfsprekend gaat er ook een woord van dank uit naar mijn collega's bij de vakgroep BW, waar ik tot op heden met veel plezier gewerkt heb. In het bijzonder wil ik Lars, René, Marchel, Gerard R., Mirjam, Mariëlle, Mascha en Henny als (ex)mede-AlO's noemen. Tot de prettige werksfeer hebben zeker ook bijgedragen de inmiddels als "zeergeleerden" vertrokken ex-AlO's; Harold, Jos V., en Gerard H. De koffiekamer- en kroegdiscussies waren zonder uitzondering 
zinloos, oeverloos en van een bijzonder wetenschappelijk gehalte. Waarschijnlijk daarom is een periodiek AlO-overleg zo ontspannend. Voor aanstaande promovendi heb ik nog 22 ongebruikte stellingen liggen die hun oorsprong ergens in de binnenstad van Maastricht hebben. Van deze stellingen zijn stelling 6 'Gors rules' en stelling 18 'Gors still rules' eventueel bruikbaar maar moeilijk verdedigbaar. Lars Borghouts, kamergenoot en paranymf, wil ik bedanken voor de plezierige wijze waarop we samen vele uren in onze helpdesk doorgebracht hebben. Als Jeff Buckley zo kon zwemmen als muziek maken, zouden we nog meer plezier hebben van onze CD-speler. Collega's van de buurvakgroep humane biologie, met name Joan, Olav, Gerrit van H. en Patrick S. wil ik bedanken voor de zinvolle en zinloze discussies. Asker, ik denk nog vaak met plezier terug aan de eerste schreden die we samen op het wetenschappelijke pad gezet hebben. Je aanstaande vertrek naar Birmingham zal het aantal gezamelijke fietstochtjes beperken, het moet echter geen belemmering zijn om onze vriendschap voort te zetten en onze gezamelijke onderzoeks activiteiten uit te bereiden. Jan de Louw, Mark Geusen, Peter Hollands, Roger vdB, Jolanda van Dongen en Suzanne Wiertsema zijn stage studenten aan wie ik dank verschuldigd ben voor hun bijdragen aan dit proefschrift. Jan, ik heb veel van je geleerd in de periode dat je bij ons gewerkt hebt. Je vermogen om complexe regeltechniek aan een niet techneut uit te leggen duidt op een groot didactisch vermogen. Gelukkig ben je, net als meerdere AlO's bij BW, een liefhebber van yoghurt met zwoerd. Jolanda, de ontwikkeling van een goed duurprotocol is complexer gebleken dan ik had ingeschat, niettemin hoop ik dat je voldoening vindt in het feit dat de data van jou experimenten dankbaar gebruikt zijn in hoofdstuk 2 van dit proefschrift. Suzanne, je was mijn eerste stagiaire. Ondanks de productieve tijd en prettige samenwerking ziijn de data uit jou experimenten helaas niet in dit proefschrift beland. Peter en Roger, vanzelfsprekend herkennen jullie in dit proefschrift precies die plaatjes waar jullie de coupes voor gesneden hebben. Zonder Karel-Joep Hommels is het de vraag of Fig 2.1 begrijpelijk geworden zou zijn, Kjoep, bedankt.

Inmiddels is de motregen overgegaan in plensbuien en besluit ik niet over de Planck terug te rijden maar voor de afkorting door de Voerstreek te kiezen, de zogenaamde hypo-route. Dat doet me eraan denken dat ik op deze plek ook mijn fietsmaten Ronald, Arno, en broer en tevens paranymf Reinout will bedanken voor de vele prettige en ontspannende fietsuren en samenwerking. Reinout, ook bedankt voor het simpele feit dat je er gewoon af en toe was (bent).

Diegenen die tevergeefs hun naam in dit dankwoord gezocht hebben moeten contact opnemen met het belgisch meteorologisch instituut te Ukkel dat voor deze ochtend droog weer, en dus een veel langer rondje met eventueel meer namen, in het vooruitzicht had gesteld. 


\section{Curriculum Vitae}

Matthijs Hesselink werd op 8 augustus 1968 te Bemmel geboren. Hij is opgegroeid in Enschede alwaar hij tussen 1980 en 1987 het Atheneum doorlopen heeft op het St. Jacobus College. In 1987 is hij begonnen met de studie gezondheidswetenschappen aan de Universiteit Maastricht, afstudeerrichting bewegingswetenschappen. Deze studie heeft hij in 1991 succesvol afgerond. In 1991 heeft hij o.l.v. Dr. J. O'Hanlon gefungeerd als onderzoeks assistent bij het toenmalige instituut voor geneesmiddelen veiligheid en gedrag (IVGV) te Maastricht. In de eerste helft van 1992 heeft hij als inspanningstysioloog inspanningstesten verricht bij General Electric Plastics in Bergen op Zoom. In de tweede helft van 1992 is hij als test assistent verbonden geweest aan de vakgroep neuropsychologie, Universiteit Maastricht, waar hij onder supervisie van Dr. W.J. Riedel testwerk verrichtte t.b.v. het project 'Cognitive (dys)function and cycloserin in age associated memory impairment'. Eind December 1992 is hij als AlO in dienst getreden bij de vakgroep bewegingswetenschappen, Universiteit Maastricht. Dit proefschrift is de verslaglegging van het onderzoek dat hij gedurende zijn AlO-periode heeft verricht. In 1995 was hij geselecteerd deelnemer aan de cursus quantitative immunoelectron microscopy', Dundee University, Schotland, georganiseerd door de European Molecular Biology Organization (EMBO). Vanaf October 1997 tot heden is hij als universitair docent werkzaam bij de vakgroep bewegingswetenschappen te Maastricht. 


\section{Publications}

\section{Full papers in peer reviewed journals}

- Cheng, B., H. Kuipers, A. C. Snyder, H. A. Keizer, A. Jeukendrup, and M. Hesselink. A new approach for the determination of ventilatory and lactate thresholds. Int J Sports Med 13: 518-522, 1992.

- Hesselink, M. K. C., H. Kuipers, P. Geurten, and H. van Straaten. Structural muscle damage and muscle strength after incremental number of isometric and forced lengthening contractions. J Muscle Res Cell Motii 17: 335-341, 1996.

- Hesselink, M. K. C., H. Kuipers, H. A. Keizer, M. R. Drost, and G. J. van der Vusse. Acute and sustained effects of isometric and lengthening muscle contractions on high-energy phosphates and glycogen metabolism in rat tibialis anterior muscle. J Muscle Res and Cell Motilaccepted, 1997.

- Hesselink, M. K. C. L. B. Borghouts, H. A. Keizer, and H. Kuipers. Immunolocalization of GLUT4 at light microscopical level in rat cardiac and white gastrocnemius muscle. Submitted 1997.

- Hesselink, M. K. C., M. R. Drost, ل. Komulainen, R. Uotila, H. A. Keizer, and H. Kuipers. Work during lengthening contractions of rat dorsiflexor muscles previously subjected to lengthening or shortening contractions. Submitted 1997.

- Hesselink, M. K. C., H. A. Keizer, M. R. Drost, Y. F. de Jong, and H. Kuipers. Muscle glycogen following lengthening and shortening muscle contractions in relation to activity of glycogen regulatory enzymes and muscle GLUT4 content. Submitted' 1997.

- Jeukendrup, A., M. K. C. Hesselink, A. C. Snyder, H. Kuipers, and H. A. Keizer. Physiological changes in male competitive cyclists after two weeks of intensified training. Int J Sports Med 13: 534$541,1992$.

- Jeukendrup, A. E., and M. K. C. Hesselink. Overtraining; what do lactate curves tell us? Br J Sports Med'28, 1994.

- Rooyackers, O. E., M. K. C. Hesselink, and A. J. M. Wagenmakers. Contraction tailure of skeletal muscle of rats recovering from critical illiness. Clinical Science. Feb 92: 189-195, 1997.

- Snyder, A. C., A. E. Jeukendrup, M. K. C. Hesselink, H. Kuipers, and C. Foster. A physiological/psychological indicator of over-reaching during intensive training. Int J Sports Med 14: 2932, 1993.

\section{Published abstracts}

- Hesselink, M. K. C., M. R. Drost, and H. Kuipers. Increased external work during forced lengthening contractions of muscles previously subjected to damaging. Med Sci Sports and Exerc 29: S66, 1997.

- Hesselink, M. K. C., E. van Breda, P. Geurten, H. Kuipers, and H. A. Keizer. Immunalacalization of GLUT4 in rat gastrocnemius muscle by light microscopy. Eur J Physio/ 430: R69, 1995.

- Hesselink, M. K. C., M. R. Drost, and H. Kuipers. On-line measurement of peak torque of rat dorsiflexor muscles during forced lengthening contractions. Eur J Physiol 430: R56, 1995.

- Hesselink, M. K. C., E. Koerts-de Lang, M. R. Drost, and A. M. W. J. Schols. Work of rat dorsiflexor hindlimb muscles after 2-week corticosteroid treatment. The Physiologist 39: A-58, 1996. 
- Hesselink, M. K. C., G. van Kranenburg, and H. Kuipers. Muscle glycogen content and related enzymes immediately and 24 hours after isometric and forced lengthening contractions. Med Sci Sponts Exerc 27: $\$ 3,1995$.

- Hesselink, M.K. C. H. Kuipers, and P. Geurten. Isometric torque of rat dorsiftexor muscle after incremental number of isometric and eccentric contractions. J Physiol London 479: 156, 1994.

- Hesselink, M. K. C. H. Kuipers, and P. Geurten. Number of forced lengthening contractions and amount of skeletal muscle damage. Med Sci Sports Exerc 26: S124, 1994.

- Hessellink, M. K. C., H. Kuipers, P. Geurten, W. A. Coumans, and G. J. van der Vusse. ATP content of rat tibialis anterior muscle immediately and 24 hours after eccentric and isometric exercise. Eur $J$ Appl Physiol 69: S11, 1994.

- Keizer, H. A., M. K. C. Hesselink, and H. Kuipers Expression of GLUT4 in skeletal muscle microvascular endothelium. Eur J Physiol 430: R152, 1995.

- Koerts-de Lang, E., G. J. van der Vusse, M. K. C. Hesselink, E. F. M. Wouters, and A. M. W. J. Schols. Effects of prednisolone, triamcinolone and nutritional deprivation on enzyme capacity of rat tibialis anterior muscle. Am J Resp Crit Car Med 155: A922, 1997.

- Komulainen, J., M. K. C. Hesselink, H. Kuipers, and V. Vihko. Forced lengthening contractions and muscle fiber injury. Med Sci Sports Exerc 28: S188, 1996.

- Komulainen, J., R. Uotila, M. K. C. Hesselink, H. Kuipers, S. Koskinen, T. E. S. Takala, and V. Vihko. The damaging effect of forced lengthening contractions on skeletai muscle is followed by fiber hypertrophy. Biochemistry of Exercise Congress, Sydney, 1997.

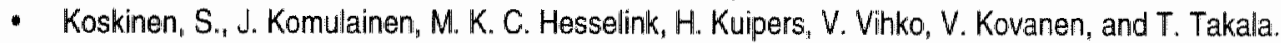
Gelatinolytic enzyme activities in skeletal muscles subjected to forced eccentric contractions. Biochemistry of Exercise Congress, Sydney, 1997.

- Koskinen, S. O. A., V. Kovanen, J. Komulainen, M. K. C. Hesselink, H. Kuipers, V. Vihko, and T. E. S. Takala. Type IV collagen, MMP-2, and TIMP-2 mPNA levels in skeletal muscle subjected to forced eccentric contractions. Med Sci Sports Exerc 28: S153, 1996.

- Kuipers, H., M. K. C. Hesselink, W. A. Coumans, and G. J. van der Vusse. Adenine nucleotide degradation and IMP production in rat tibialis anterior muscle after isometric and forced lengthening exercise. Med Sci Sports Exerc 27: S220, 1995.

- Rooyackers, O. E., M. K. C. Hesselink, and A. J. M. Wagenmakers. Impaired energy metabolism in muscle of zymosan treated rats recovering from critical illiness. Clin Nutr 13: 6, 1994.

- Snyder, A. C. A. E. Jeukendrup, M. K. C. Hesselink, and H. Kuipers. A physiological/psychological indicator of overtraining. The Physiologist 35: 1B1, 1992.

- Takala, T. E. S., S. O. A. Koskinen, J. Komulainen, M. K. C. Hesselink, V. Vihko, H. Kuipers, and $\checkmark$, Kovanen. Eccentric contractions increase expression of fibrillar collagens, prolyl 4-hydroxylase and lysyl oxidase in skeletal muscle. Med Sci Sports Exerc 28: S153, 1996.

- Takala, T. E. S., S. O. A. Koskinen, R. Uotila, M. K. C. Hesselink, H. Kuipers, V. Vihko, V. Kovanen, and J. Komulainen. Effects of forced eccentric and concentric contractions on prolyl 4hydroxylase activity in skelelal muscle. Biochemistry of Exercise Congress, Sydney, 1997. 